Pacific

Journal of

Mathematics

GREEN CURRENTS FOR MODULAR CYCLES IN ARITHMETIC QUOTIENTS OF COMPLEX HYPERBALLS

MASAo TsUZUKI

Volume $227 \quad$ No. 2

October 2006 


\title{
GREEN CURRENTS FOR MODULAR CYCLES IN ARITHMETIC QUOTIENTS OF COMPLEX HYPERBALLS
}

\author{
MASAO TSUZUKI
}

\begin{abstract}
We obtain a Green current in the sense of Gillet-Soulé on an arithmetic quotient of a complex hyperball for the modular cycle stemming from a complex subhyperball of codimension greater than one, generalizing the classical construction of the automorphic Green function for the modular curves.
\end{abstract}

\section{Introduction and basic notation}

Let $X$ be a complex manifold and $Y$ its analytic subvariety of codimension $r$. The Green current for $Y$ is defined to be a current $G$ of $(r-1, r-1)$-type on $X$ such that $\mathrm{dd}^{\mathrm{c}} \varphi+\delta_{Y}$ is represented by a $C^{\infty}$-form of $(r, r)$-type on $X$. In the arithmetic intersection theory developed by Gillet and Soulé, the role played by the algebraic cycles in the conventional intersection theory is replaced with the arithmetic cycles. In a heuristic sense, Green currents are regarded as the "archimedean" ingredient of such arithmetic cycles [Gillet and Soulé 1990].

Consider the case when $X$ is the quotient of a Hermitian symmetric domain $G / K$ by an arithmetic lattice $\Gamma$ in the semisimple Lie group $G$, and $Y$ is a modular cycle stemming from a modular imbedding $H / H \cap K \hookrightarrow G / K$, where $H$ is a reductive subgroup of $G$ such that $H \cap K$ is maximally compact in $H$. Inspired by the classical works on the resolvent kernel functions of the Laplacian on Riemannian surfaces [Hejhal 1983] and also by a series of works [Miatello and Wallach 1989; 1992], T. Oda posed a plan to construct a Green current for $Y$ making use of a secondary spherical function on $H \backslash G$, giving evidence for the divisorial case with some conjectures. Among many possible choices of the Green currents for a modular cycle $Y$, this construction may provide a way to fix a natural one. If $r=1$, namely $Y$ is a modular divisor, we already obtained a satisfactory result by properly introducing the secondary spherical functions [Oda and Tsuzuki 2003]. So it is quite natural to ask whether the same method works for the higher codimensional case. Here we focus on the case when $G / K$ is an $n$-dimensional complex hyperball and $H / H \cap K$ is also a complex hyperball of codimension $r>1$.

MSC2000: 14G40, 11F72.

Keywords: Green current, spherical functions, Poincaré series. 
After two preliminary sections, in the third section we introduce the vectorvalued secondary spherical function $\phi_{s}$ together with its simple characterization in Theorem 3.2. Then we form the Poincaré series $G_{s}(g)=\sum_{\gamma \in H \cap \Gamma \backslash \Gamma} \phi_{s}(\gamma g)$ which is shown in Theorem 4.2 to be $L^{1}$-convergent to yield an $(r-1, r-1)$-current on $\Gamma \backslash G / K$ when the parameter $s$ lies in some right-half plane. In order to analyze $G_{s}$ further, in Section 5 we study its derivatives $\left(-\frac{1}{2 s} \frac{d}{d s}\right)^{j} G_{s}$ with sufficiently large $j$ by the same technique used in [Gon and Tsuzuki 2002]. Through the inductive argument of Theorem 6.2, we show in Theorem 6.3 that the function $s \mapsto G_{s}$ has a meromorphic continuation to the whole $s$-plane with a functional equation and has a simple pole at the point $s=n-2 r+2$. We put $\mathscr{G}$ to be the constant term of the Laurent expansion of $G_{s}$ at $s=n-2 r+2$ and prove in Theorem 6.5 that the $(r-1, r-1)$-current $\mathscr{G}$ is a Green current for our $Y$. For that purpose we introduce another $(r, r)$-current $\Psi_{s}$ as a suitable Poincaré series and study its properties. Among other things we show that the value of $\Psi_{s}$ at its regular point $s=n-2 r+2$ is square-integrable harmonic form representing the current $\mathrm{dd}^{\mathrm{c}} \varphi g+\delta_{D}$ up to a constant multiple; see Theorem 7.5.

This paper is a continuation of the joint work of Professor Takayuki Oda and the author [Oda and Tsuzuki 2003]. Thanks are due to Professor Takayuki Oda for his interest in this work and fruitful discussions.

\section{Preliminaries}

Let $n$ and $r$ be integers such that $2 \leqslant r<n / 2$. For a matrix $X=\left(x_{i j}\right) \in \mathrm{M}_{n}(\mathbb{C})$, $X^{*}$ denotes its conjugate transpose $\left(\bar{x}_{j i}\right)$. Consider the two involutions $\sigma$ and $\theta$ of the Lie group $G=\mathrm{U}(n, 1):=\left\{g \in \mathrm{GL}_{n+1}(\mathbb{C}) \mid g^{*} \mathrm{I}_{n, 1} g=\mathrm{I}_{n, 1}\right\}$ defined by $\theta(g)=\mathrm{I}_{n, 1} g \mathrm{I}_{n, 1}$ and $\sigma(g)=\mathrm{S} g \mathrm{~S}$ respectively. Here $\mathrm{I}_{n, 1}:=\operatorname{diag}\left(\mathrm{I}_{n},-1\right)$ and $\mathrm{S}=\operatorname{diag}\left(\mathrm{I}_{n-r},-\mathrm{I}_{r}, 1\right)$. Then $K:=\{g \in G \mid \theta(g)=g\} \cong \mathrm{U}(n) \times \mathrm{U}(1)$ is a maximal compact subgroup in $G$ and $H:=\{g \in G \mid \sigma(g)=g\} \cong \mathrm{U}(n-r, 1) \times \mathrm{U}(r)$ is a symmetric subgroup of $G$ such that $K_{H}:=H \cap K \cong \mathrm{U}(n-r) \times \mathrm{U}(r) \times \mathrm{U}(1)$ is maximally compact in $H$.

The group $G$ acts on the hyperball $\mathfrak{D}=\left\{z=\left.{ }^{t}\left(z_{1}, \ldots, z_{n}\right) \in \mathbb{C}^{n}\left|\sum_{i=1}^{n}\right| z_{i}\right|^{2}<1\right\}$ transitively by the fractional linear transformation

$$
g \cdot z=\frac{g_{11} z+g_{12}}{g_{21} z+g_{22}}, \quad g=\left[\begin{array}{ll}
g_{11} & g_{12} \\
g_{21} & g_{22}
\end{array}\right] \in G, z \in \mathbb{C}^{n} .
$$

Under the identification $G / K \cong \mathfrak{D}$ of $G$-manifolds sending $g K$ to $g \cdot 0$, the quotient $H / K_{H}$ corresponds to the $H$-orbit of 0 , i.e., $\mathfrak{D}^{H}:=\left\{z \in \mathfrak{D} \mid z_{n-r+1}=\cdots=z_{n}=0\right\}$. In particular the real codimension of $H / K_{H}$ in $G / K$ is $2 r$.

The Lie algebra $\mathfrak{g}:=\operatorname{Lie}(G)$ is realized in its complexification $\mathfrak{g}_{\mathbb{C}}=\mathfrak{g l}_{n+1}(\mathbb{C})$ as an $\mathbb{R}$-subalgebra of all $X \in \mathfrak{g l}_{n+1}(\mathbb{C})$ such that $X^{*} \mathrm{I}_{n, 1}+\mathrm{I}_{n, 1} X=0_{n+1}$. Let $\mathfrak{p}$ 
be the orthogonal complement of $\mathfrak{k}:=\operatorname{Lie}(K)$ in $\mathfrak{g}$ with respect to the $G$-invariant, nondegenerate $\mathbb{R}$-bilinear form $\langle X, Y\rangle=2^{-1} \operatorname{tr}(X Y)$ on $\mathfrak{g}$. We have the Cartan decomposition $\mathfrak{g}=\mathfrak{k} \oplus \mathfrak{p}$. We put $G / K$ a unique $G$-invariant complex structure such that the map $G / K \cong \mathfrak{D}$ is biholomorphic.

For $1 \leqslant i, j \leqslant n+1$, let $\mathrm{E}_{i, j}:=\left(\delta_{u i} \delta_{v j}\right)_{u v} \in \mathfrak{g l}_{n+1}(\mathbb{C})$ be the matrix unit. Put

$$
\begin{aligned}
& X_{i}:=\mathrm{E}_{i, n+1} \text { and } \bar{X}_{i}=\mathrm{E}_{n+1, i}, \quad 1 \leqslant i \leqslant n-1 ; \\
& X_{0}:=\mathrm{E}_{n, n+1} \text { and } \bar{X}_{0}=\mathrm{E}_{n+1, n} .
\end{aligned}
$$

Then $\mathfrak{p}_{+}=\sum_{i=0}^{n-1} \mathbb{C} X_{i}$ corresponds to the holomorphic tangent space of $G / K$ at $K$, and $\mathfrak{p}_{-}=\sum_{i=0}^{n-1} \mathbb{C} \bar{X}_{i}$ to the antiholomorphic tangent space.

The exterior algebra $\bigwedge \mathfrak{p}_{\mathbb{C}}^{*}$ is decomposed to the direct sum of subspaces

$$
\bigwedge^{p, q} \mathfrak{p}_{\mathbb{C}}^{*}:=\left(\bigwedge^{p} \mathfrak{p}_{+}^{*}\right) \otimes\left(\bigwedge^{q} \mathfrak{p}_{-}^{*}\right), \quad p, q \in \mathbb{N} .
$$

With $\left\{\omega_{i}\right\}$ the basis of $\mathfrak{p}_{+}^{*}$ dual to $\left\{X_{i}\right\}$ and $\left\{\bar{\omega}_{i}\right\}$ the basis of $\mathfrak{p}_{-}^{*}$ dual to $\left\{\bar{X}_{i}\right\}$, put

$$
\omega:=\frac{\sqrt{-1}}{2} \sum_{i=0}^{n-1} \omega_{i} \wedge \bar{\omega}_{i} \in \bigwedge^{1,1} \mathfrak{p}_{\mathbb{C}}^{*} \cap \wedge \mathfrak{p}^{*} \quad \text { and } \quad \text { vol }:=\frac{1}{n !} \omega^{n} \in \bigwedge^{n, n} \mathfrak{p}_{\mathbb{C}}^{*} \cap \wedge \mathfrak{p}^{*}
$$

The dual inner product on $\mathfrak{p}^{*}$ naturally extends to the Hermitian inner product $(\cdot \mid \cdot)$ of $\bigwedge \mathfrak{p}_{\mathbb{C}}^{*}$. The Hodge star operator $*$ is the $\mathbb{C}$-linear automorphism of $\bigwedge \mathfrak{p}_{\mathbb{C}}^{*}$ such that $* \bar{\alpha}=\overline{* \alpha}$ and $(\alpha \mid \beta)$ vol $=\alpha \wedge * \bar{\beta}$, for $\alpha, \beta \in \wedge \mathfrak{p}_{\mathbb{C}}^{*}$. We remark that

$$
\left\{X_{j}+\bar{X}_{j}, \sqrt{-1}\left(X_{j}-\bar{X}_{j}\right)\right\}_{j=0}^{n-1}
$$

is an orthonormal basis of $\mathfrak{p}$, dual to $\left\{2^{-1}\left(\omega_{j}+\bar{\omega}_{j}\right),-2^{-1} \sqrt{-1}\left(\omega_{j}-\bar{\omega}_{j}\right)\right\}_{j=0}^{n-1}$. For $\alpha \in \wedge \mathfrak{p}_{\mathbb{C}}^{*}$, define the endomorphism $e(\alpha): \wedge \mathfrak{p}_{\mathbb{C}}^{*} \rightarrow \wedge \mathfrak{p}_{\mathbb{C}}^{*}$ by $e(\alpha) \beta=\alpha \wedge \beta$. As usual, we have the Lefschetz operator $L:=e(\omega)$ and its adjoint operator $\Lambda=e^{*}(\omega)$ acting on the finite dimensional Hilbert space $\wedge \mathfrak{p}_{\mathbb{C}}^{*}$ [Wells 1980 , Chapter V].

Put $\mathfrak{h}=\operatorname{Lie}(H)$. Then $\theta$ restricts to a Cartan involution of $\mathfrak{h}$ giving the decomposition $\mathfrak{h}=(\mathfrak{h} \cap \mathfrak{k}) \oplus(\mathfrak{h} \cap \mathfrak{p})$. The complex structure of $\mathfrak{p}$ induces that of $\mathfrak{h} \cap \mathfrak{p}$ giving the decomposition $(\mathfrak{h} \cap \mathfrak{p})_{\mathbb{C}}=(\mathfrak{h} \cap \mathfrak{p})_{+} \oplus(\mathfrak{h} \cap \mathfrak{p})_{-}$with

$$
(\mathfrak{h} \cap \mathfrak{p})_{+}=\mathfrak{h}_{\mathbb{C}} \cap \mathfrak{p}_{+}=\sum_{i=1}^{n-r} \mathbb{C} X_{i} \quad \text { and } \quad(\mathfrak{h} \cap \mathfrak{p})_{-}=\mathfrak{h}_{\mathbb{C}} \cap \mathfrak{p}_{-}=\sum_{i=1}^{n-r} \mathbb{C} \bar{X}_{i} .
$$

We introduce two tensors $\omega_{H}$ and $\eta$ as

$\omega_{H}:=\frac{\sqrt{-1}}{2} \sum_{i=1}^{n-r} \omega_{i} \wedge \bar{\omega}_{i} \quad$ and $\quad \eta:=\frac{\sqrt{-1}}{2} \sum_{j=n-r+1}^{n-1} \omega_{i} \wedge \bar{\omega}_{i}=\omega-\omega_{H}-\frac{\sqrt{-1}}{2} \omega_{0} \wedge \bar{\omega}_{0}$. 
For $0 \leqslant p \leqslant r-1$ let $S_{p}$ denote the set of all subsets $J \subset\{n-r+1, \ldots, n-1\}$ such that $\sharp(J)=p$. Then a computation shows that

$$
\eta^{p}=\left(\frac{\sqrt{-1}}{2}\right)^{p} p ! \sum_{J \in S_{p}} \prod_{j \in J} \omega_{j} \wedge \bar{\omega}_{j}, \quad 0 \leqslant p \leqslant r-1 .
$$

From [Wells 1980, (1.5), p. 163], we have

$$
\Lambda\left(\eta^{p}\right)=p(r-p) \eta^{p-1}, \quad 0 \leqslant p \leqslant r-1 .
$$

The coadjoint representation of $K$ on $\mathfrak{p}^{*}$ is extended to the unitary representation $\tau: K \rightarrow \mathrm{GL}\left(\wedge \mathfrak{p}_{\mathbb{C}}^{*}\right)$ in such a way that $\tau(k)(\alpha \wedge \beta)=\tau(k) \alpha \wedge \tau(k) \beta$ holds for all $\alpha, \beta \in \wedge \mathfrak{p}_{\mathbb{C}}^{*}$ and $k \in K$. The differential of $\tau$ is also denoted by $\tau$. Then we have

$$
\begin{aligned}
& \tau(Z)(\alpha \wedge \beta)=(\tau(Z) \alpha) \wedge \beta+\alpha \wedge(\tau(Z) \beta), \\
& (\tau(Z) \alpha \mid \beta)=-(\alpha \mid \tau(\bar{Z}) \beta)
\end{aligned}
$$

for $\alpha, \beta \in \wedge \mathfrak{p}_{\mathbb{C}}^{*}, Z \in \mathfrak{k}_{\mathbb{C}}$.

The irreducible decomposition of the $K$-invariant subspaces $\wedge^{p, q} \mathfrak{p}_{\mathbb{C}}^{*}$ is wellknown.

Lemma 1.1. Let $p, q$ be nonnegative integers such that $p+q \leqslant n$. Put

$$
F_{p, q}:=\left\{\alpha \in \bigwedge^{p, q} \mathfrak{p}_{\mathbb{C}}^{*} \mid \Lambda(\alpha)=0\right\} .
$$

Then $F_{p, q}$ is an irreducible $K$-invariant subspace of $\wedge \mathfrak{p}_{\mathbb{C}}^{*}$. The $K$-homomorphism L induces a linear injection $\bigwedge^{p-1, q-1} \mathfrak{p}_{\mathbb{C}}^{*} \rightarrow \bigwedge^{p, q} \mathfrak{p}_{\mathbb{C}}^{*}$ whose image is the orthogonal complement of $F_{p, q}$ in $\bigwedge^{p, q} \mathfrak{p}_{\mathbb{C}}^{*}$. In other words,

$$
\bigwedge^{p, q} \mathfrak{p}_{\mathbb{C}}^{*}=F_{p, q} \oplus L\left(\bigwedge^{p-1, q-1} \mathfrak{p}_{\mathbb{C}}^{*}\right) .
$$

Proof. Use [Borel and Wallach 1980, Lemma 4.9, p. 199].

The one dimensional $\mathbb{R}$-subspace $\mathfrak{a}=\mathbb{R} Y_{0}$ with $Y_{0}:=X_{0}+\bar{X}_{0} \in \mathfrak{p}$ is a maximal abelian subalgebra in $\mathfrak{q} \cap \mathfrak{p}$ with $\mathfrak{q}$ the $(-1)$-eigenspace of $d \sigma$, the differential of $\sigma$. Since $(G, H)$ is a symmetric pair, by the general theory [Heckman and Schlichtkrull 1994, Theorem 2.4, p. 108], the group $G$ is a union of double cosets $H a_{t} K(t \geqslant 0)$ with

$$
a_{t}:=\exp \left(t Y_{0}\right)=\operatorname{diag}\left(\mathrm{I}_{n-1},\left[\begin{array}{cc}
\cosh t & \sinh t \\
\sinh t & \cosh t
\end{array}\right]\right), \quad t \in \mathbb{R} .
$$

Put $A=\left\{a_{t} \mid t \in \mathbb{R}\right\}$. Let $M_{0}$ be the group of all the elements $k \in K$ such that $\operatorname{Ad}(k) Y_{0}=Y_{0}$ and set $M=M_{0} \cap H$. Then

$$
\begin{aligned}
& M_{0}=\left\{\operatorname{diag}\left(u, u_{0}, u_{0}\right) \mid u \in \mathrm{U}(n-1), u_{0} \in \mathrm{U}(1)\right\}, \\
& M=\left\{\operatorname{diag}\left(u_{1}, u_{2}, u_{0}, u_{0}\right) \mid u_{1} \in \mathrm{U}(n-r), u_{2} \in \mathrm{U}(r-1), u_{0} \in \mathrm{U}(1)\right\} .
\end{aligned}
$$


It is important to know the structure of the $M$-fixed part of $F_{p, p}$.

Proposition 1.2. Let $p$ be an integer such that $0<p \leqslant r$. Put

$$
\begin{aligned}
& \mathrm{v}_{0}^{(p)}=\frac{1}{n-p+1} \sum_{j=0}^{p} c_{j}^{(p)} L^{p-j}\left((n-p-j+1) \eta^{j}+\frac{\sqrt{-1}}{2} j(r-j) \omega_{0} \wedge \bar{\omega}_{0} \wedge \eta^{j-1}\right), \\
& \mathrm{v}_{1}^{(p)}=\frac{-1}{p(n-2 p+1)} \sum_{j=0}^{p} c_{j}^{(p)} L^{p-j}\left((p-j) \eta^{j}+\frac{\sqrt{-1}}{2} j(r-j) \omega_{0} \wedge \bar{\omega}_{0} \wedge \eta^{j-1}\right),
\end{aligned}
$$

where

$$
c_{j}^{(p)}=(-1)^{j}\left(\begin{array}{c}
p \\
j
\end{array}\right)\left(\begin{array}{c}
n-p+1 \\
j
\end{array}\right)\left(\begin{array}{c}
r-1 \\
j
\end{array}\right)^{-1}, 0 \leqslant j \leqslant \inf (p, r-1), \quad \text { and } \quad c_{r}^{(r)}=1 .
$$

Then $F_{p, p}^{M}$ is a two-dimensional space generated by $v_{0}^{(p)}$ and $v_{1}^{(p)}$.

Proof. Let $W$ be the $\mathbb{C}$-span of elements $\omega_{i}, \bar{\omega}_{i}(1 \leqslant i \leqslant n-1)$. Then for each $p, q$, $\wedge^{p, q} \mathfrak{p}_{\mathbb{C}}^{*}$ is an orthogonal direct sum of four subspaces $\wedge^{p, q} W, e\left(\omega_{0}\right)\left(\bigwedge^{p-1, q} W\right)$, $e\left(\bar{\omega}_{0}\right)\left(\wedge^{p, q-1} W\right)$ and $e\left(\omega_{0} \wedge \bar{\omega}_{0}\right)\left(\wedge^{p-1, q-1} W\right)$. The space $\wedge W$ has the Lefschetz operator $L_{0}$ and its adjoint $\Lambda_{0}$. These are also given as $L_{0}=e\left(\omega_{H}+\eta\right) \mid \wedge W$ and $\Lambda_{0}=\Lambda \mid \wedge W$. Let $p, q$ be natural numbers such that $p+q<n$. Then the subspace $E_{p, q}=\left\{\beta \in \bigwedge^{p, q} W \mid \Lambda_{0}(\beta)=0\right\}$ is an irreducible $M_{0}$-subspace of $\wedge W$; see Lemma 1.1. First we show that the linear map $\mathscr{T}^{p, q}$ from the direct sum $E_{p, q} \oplus$ $E_{p-1, q} \oplus E_{p, q-1} \oplus E_{p-1, q-1}$ to $\bigwedge \mathfrak{p}_{\mathbb{C}}^{*}$ defined by sending $\left(\beta_{1}, \beta_{2}, \beta_{3}, \xi\right)$ to $\mathscr{T}^{p, q}\left(\beta_{1}, \beta_{2}, \beta_{3}, \xi\right)$

$$
=\beta_{1}+\left(\beta_{2} \wedge \omega_{0}\right)+\left(\beta_{3} \wedge \bar{\omega}_{0}\right)+\frac{\sqrt{-1}}{2} \xi \wedge \omega_{0} \wedge \bar{\omega}_{0}-\frac{L_{0}(\xi)}{n-p-q+1}
$$

is an $M_{0}$-isomorphism onto $F_{p, q}$.

An element $\zeta \in \bigwedge^{p, q} \mathfrak{p}_{\mathbb{C}}^{*}$ is expressed uniquely using an element $\left(\zeta_{1}, \beta_{2}, \beta_{3}, \zeta_{4}\right)$ in the direct sum $\wedge^{p, q} W \oplus \bigwedge^{p-1, q} W \oplus \wedge^{p, q-1} W \oplus \wedge^{p-1, q-1} W$ as the sum $\zeta=$ $\zeta_{1}+\left(\beta_{2} \wedge \omega_{0}\right)+\left(\beta_{3} \wedge \bar{\omega}_{0}\right)+\left(\zeta_{4} \wedge \omega_{0} \wedge \bar{\omega}_{0}\right)$. We examine the condition $\Lambda(\zeta)=0$. Since the four equalities $\Lambda\left(\zeta_{1}\right)=\Lambda_{0}\left(\zeta_{1}\right), \Lambda\left(\beta_{2} \wedge \omega_{0}\right)=\Lambda_{0}\left(\beta_{2}\right) \wedge \omega_{0}, \Lambda\left(\beta_{3} \wedge \bar{\omega}_{0}\right)=$ $\Lambda_{0}\left(\beta_{3}\right) \wedge \bar{\omega}_{0}$ and $\Lambda\left(\zeta_{4} \wedge \omega_{0} \wedge \bar{\omega}_{0}\right)=\Lambda_{0}\left(\zeta_{4}\right) \wedge \omega_{0} \wedge \bar{\omega}_{0}+\frac{2}{\sqrt{-1}} \zeta_{4}$ hold, the condition $\Lambda(\zeta)=0$ is equivalent to $\Lambda_{0}\left(\beta_{2}\right)=\Lambda_{0}\left(\beta_{3}\right)=\Lambda_{0}\left(\zeta_{4}\right)=0$ and $\zeta_{4}=-\frac{\sqrt{-1}}{2} \Lambda_{0}\left(\zeta_{1}\right)$. We can write $\zeta_{1}=\beta_{1}-(n-p-q+1)^{-1} L_{0}(\xi)$ with $\beta_{1} \in E_{p, q}$ and $\xi \in \wedge^{p-1, q-1} W$ uniquely; see Lemma 1.1. By [Wells 1980, Proposition 1.1(c), p. 160], we have $\Lambda_{0} L_{0}(\xi)=(n-p-q+1) \xi$ and hence $\zeta_{4}=\frac{\sqrt{-1}}{2} \xi$. Since $\Lambda_{0}\left(\zeta_{4}\right)=0, \xi \in E_{p-1, q-1}$. Consequently we arrive at $\zeta=\mathscr{T}^{p, q}\left(\beta_{1}, \beta_{2}, \beta_{3}, \xi\right)$ to know the image of $\mathscr{T}^{p, q}$ is $F_{p, q}$. The injectivity of $\mathscr{T}^{p, q}$ follows from $\operatorname{Im} L_{0} \cap E_{p, q}=\{0\}$. Since $\omega_{0}, \bar{\omega}_{0}$ are $M_{0}$-invariant and since $L_{0}$ is an $M_{0}$-homomorphism, the map $\mathscr{T}^{p, q}$ is also an $M_{0}$-homomorphism. 
The map $\mathscr{T}^{p, p}$ induces a linear isomorphism

$$
E_{p, p}^{M} \oplus E_{p-1, p}^{M} \oplus E_{p, p-1}^{M} \oplus E_{p-1, p-1}^{M} \cong F_{p, p}^{M}
$$

of the $M$-invariant part.

Claim: $E_{p, q}^{M}=\{0\}$ if $p \neq q, p+q<n$. For $0 \leqslant p \leqslant r, E_{p, p}^{M}=\mathbb{C}_{u^{(p)}} \neq\{0\}$, where

$$
\mathrm{u}^{(p)}=\sum_{j=0}^{p} \frac{n-p-j+1}{n-p+1} c_{j}^{(p)} L_{0}^{p-j}\left(\eta^{j}\right) .
$$

Granting this, we easily prove the proposition easily. Indeed, a direct computation yields $\mathrm{v}_{0}^{(p)}=\mathscr{T}^{p, p}\left(\mathrm{u}^{(p)}, 0,0,0\right)$ and $\mathrm{v}_{1}^{(p)}=\mathscr{T}^{p, p}\left(0,0,0, \mathrm{u}^{(p-1)}\right)$. The claim, together with the decomposition (3) implies these tensors form a basis of $F_{p, p}^{M}$.

We prove the claim. The element $\operatorname{diag}\left(\mathrm{I}_{n-1}, u_{0}, u_{0}\right)\left(u_{0} \in \mathrm{U}(1)\right)$ of $M$ acts on $E_{p, q}$ by the scalar $u_{0}^{-p+q}$. Hence $E_{p, q}^{M}=\{0\}$ if $p \neq q$.

From now on, let $0 \leqslant p \leqslant r$. Since $M$ is a symmetric subgroup of $M_{0}$ and $E_{p, p}$ is an irreducible $M_{0}$-module, we have $\operatorname{dim}_{\mathbb{C}}\left(E_{p, p}^{M}\right) \leqslant 1$. Hence to show that $E_{p, p}^{M}=\mathbb{C} \mathrm{u}^{(p)} \neq\{0\}$, it is sufficient to prove that the element $\mathrm{u}^{(p)}$ is $M$-invariant, nonzero and $\Lambda_{0}\left(\mathrm{u}^{(p)}\right)=0$.

Since $\eta$ is $M$-invariant and $L_{0}$ is an $M_{0}$-homomorphism, the $M$-invariance of $\mathrm{u}^{(p)}$ is clear. We prove that it is nonzero using the expression

$$
\mathbf{u}^{(p)}=\sum_{\alpha=0}^{p}\left(\sum_{j=0}^{\alpha} \frac{n-p-j+1}{n-p+1} c_{j}^{(p)}\left(\begin{array}{c}
p-j \\
\alpha-j
\end{array}\right)\right) \omega_{H}^{p-\alpha} \wedge \eta^{\alpha}
$$

which we get from the original formula for $\mathrm{u}^{(p)}$ by substituting the binomial expansion of $L_{0}^{p-j}=e\left(\left(\omega_{H}+\eta\right)^{p-j}\right)$. It is easy to see that the tensors $\omega_{H}^{p-\alpha} \wedge \eta^{\alpha}$ are linearly independent. The coefficient of $\omega_{H}^{p}$ in expression (4) is $c_{0}^{(p)}$, which is nonzero. Hence we conclude $\mathrm{u}^{(p)} \neq 0$.

There remains to show that $\Lambda_{0}\left(\mathrm{u}^{(p)}\right)=0$. By [Wells 1980, Proposition 1.1(c), p. 160] and (1), we have

$$
\begin{aligned}
\Lambda_{0} L_{0}^{p-j}\left(\eta^{j}\right) & =L_{0}^{p-j} \Lambda_{0}\left(\eta^{j}\right)+(p-j)(n-j-p) L_{0}^{p-j-1}\left(\eta^{j}\right) \\
& =j(r-j) L_{0}^{p-j}\left(\eta^{j-1}\right)+(p-j)(n-j-p) L_{0}^{p-j-1}\left(\eta^{j}\right) .
\end{aligned}
$$

Hence $\Lambda_{0}\left(\mathrm{u}^{(p)}\right)$ equals $(n-p+1)^{-1}$ times

$$
\sum_{j=0}^{p-1}(n-p-j)\left((j+1)(r-j-1) c_{j+1}^{(p)}+(n-p-j+1)(p-j) c_{j}^{(p)}\right) L_{0}^{p-j-1}\left(\eta^{j}\right) .
$$

We can easily check that the coefficient of each $L_{0}^{p-j-1}\left(\eta^{j}\right)$ in the expression above is zero. 
For convenience, we put $\mathrm{v}_{0}^{(0)}=1, \mathrm{v}_{1}^{(0)}=0$; these are elements of $F_{0,0}=\mathbb{C}$.

We also need the structure of the $(H \cap K)$-invariant part of $F_{p, p}(0 \leqslant p<r)$.

Proposition 1.3. For each $0<p \leqslant r-1$, put

$$
\mathrm{w}^{(p)}=\sum_{j=0}^{p}(r-j) c_{j}^{(p)} L^{p-j}\left(\left(\omega-\omega_{H}\right)^{j}\right) .
$$

For $p=0$, we set $\mathrm{w}^{(0)}=r$. Then

$$
\mathrm{w}^{(p)}=\frac{(r-p)(n-p+1)}{n-2 p+1} \mathrm{v}_{0}^{(p)}-p(n-p-r+1) \mathrm{v}_{1}^{(p)},
$$

and $F_{p, p}^{H \cap K}=\mathbb{C}^{(p)} \neq\{0\}$.

Proof. The $(H \cap K)$-invariance of $\mathrm{w}^{(p)}$ follows from the fact that $\omega-\omega_{H}$ is $(H \cap K)$ invariant and $L$ is a $K$-homomorphism. The formula (5) follows from a direct computation. Since $H \cap K=\mathrm{U}(n-r) \times \mathrm{U}(r) \times \mathrm{U}(1)$ is a symmetric subgroup of $K=\mathrm{U}(n) \times \mathrm{U}(1)$, we have $\operatorname{dim}\left(F_{p, p}^{H \cap K}\right) \leqslant 1$. Since $\mathrm{w}^{(p)} \neq 0$ belongs to $F_{p, p}^{H \cap K}$, the last assertion follows.

For convenience, we put $\mu=r-1$ and $l=n-2 r+2$. Then our assumption $2 \leqslant r<n / 2$ is equivalent to $\mu \geqslant 1$ and $\lambda \geqslant 3$.

Proposition 1.4. Define $\mathrm{w}:=\left(\omega-\omega_{H}\right)^{\mu}$ and $\mathrm{vol}_{H}=\frac{1}{(n-r) !} \omega_{H}^{n-r}$. We have

$$
\begin{aligned}
* \operatorname{vol}_{H} & =\frac{1}{r !} \mathrm{w} \wedge\left(\omega-\omega_{H}\right), & \mathrm{w} & \wedge\left(\eta-\frac{\sqrt{-1}}{2} \mu \omega_{0} \wedge \bar{\omega}_{0}\right)=0, \\
\Lambda\left(* \operatorname{vol}_{H}\right) & =\frac{1}{\mu !} \mathrm{w}, & \mathrm{w} & =\sum_{p=0}^{\mu} \gamma_{p} L^{\mu-p}\left(\mathrm{w}^{(p)}\right),
\end{aligned}
$$

where the $\gamma_{p}, 0 \leqslant p \leqslant \mu$, are real numbers defined by the recurrence relation

$$
\gamma_{\mu}=\frac{1}{c_{\mu}^{(\mu)}}, \quad \gamma_{j} c_{j}^{(j)}=-\sum_{p=j+1}^{\mu} \gamma_{p} c_{j}^{(p)}, \quad 0 \leqslant j<\mu .
$$

Proof. Using [Wells 1980, Lemma 1.2, p. 161], we easily check the first formula in (6) by a computation. The second follows directly from $\eta^{r}=0$ and $\mathrm{w}=\eta^{\mu}+$ $\frac{\sqrt{-1}}{2} \mu \omega_{0} \wedge \bar{\omega}_{0} \wedge \eta^{\mu-1}$. The first formula in (7) is a consequence of (6) and [Wells 1980, (1.5), p. 163]. To justify the last formula, note that its right-hand side equals

$$
\sum_{j=0}^{\mu}\left(\sum_{p=j}^{\mu} \gamma_{p} c_{j}^{(p)}\right)(r-j) L^{\mu-j}\left(\omega-\omega_{H}\right)^{j} ;
$$

this equals $w$ by the definition of $w$ and $\gamma_{j}$. 


\section{Radial parts of several differential operators}

We define $2 n-1$ elements $Z_{\alpha}, \bar{Z}_{\alpha}$ and $Z_{0}$ of $\mathfrak{k}_{\mathbb{C}}$ by

$$
\begin{aligned}
& Z_{\alpha}=\mathrm{E}_{\alpha, n}, \quad \bar{Z}_{\alpha}=-\mathrm{E}_{n, \alpha}, \quad 1 \leqslant \alpha \leqslant n-1, \\
& Z_{0}=\sqrt{-1}\left(\mathrm{E}_{n, n}-\mathrm{E}_{n+1, n+1}\right) .
\end{aligned}
$$

Note that $(\mathfrak{k} \cap \mathfrak{h})_{\mathbb{C}}$ is a direct sum of $\operatorname{Lie}(M)_{\mathbb{C}}$ and the $\mathbb{C}$-span of the $2 r-1$ elements $Z_{j}, \bar{Z}_{j}(n-r+1 \leqslant j \leqslant n-1), Z_{0}$.

Lemma 2.1. For $1 \leqslant \alpha, \beta \leqslant n-1$, we have

$$
\begin{array}{ll}
\tau\left(Z_{\alpha}\right) \bar{\omega}_{\beta}=0, & \tau\left(Z_{\alpha}\right) \omega_{\beta}=-\delta_{\alpha \beta} \omega_{0}, \\
\tau\left(Z_{\alpha}\right) \bar{\omega}_{0}=\bar{\omega}_{\alpha}, & \tau\left(Z_{\alpha}\right) \omega_{0}=0, \\
\tau\left(Z_{0}\right) \omega_{\alpha}=-\sqrt{-1} \omega_{\alpha}, & \tau\left(Z_{0}\right) \omega_{0}=-2 \sqrt{-1} \omega_{0} .
\end{array}
$$

For $n-r+1 \leqslant \alpha \leqslant n-1$, we have

$$
\tau\left(Z_{\alpha}\right) \eta=-\frac{\sqrt{-1}}{2} \omega_{0} \wedge \bar{\omega}_{\alpha}, \quad \tau\left(\bar{Z}_{\alpha}\right) \eta=-\frac{\sqrt{-1}}{2} \omega_{\alpha} \wedge \bar{\omega}_{0} .
$$

Definition. Let $C_{\tau}^{\infty}$ be the space of $C^{\infty}$-functions $\varphi: G-H K \rightarrow \wedge \mathfrak{p}_{\mathbb{C}}^{*}$ having the $(H, K)$-equivariance property $\varphi(h g k)=\tau(k)^{-1} \varphi(g)$ for $h \in H, k \in K$.

Lemma 2.2. Let $i, v \in\{1, \ldots, n-r\}$ and $j, \mu \in\{n-r+1, \ldots, n-1\}$. Let $\varphi \in C_{\tau}^{\infty}$. Then:

$$
\begin{aligned}
R_{X_{i} \bar{X}_{v}} \varphi\left(a_{t}\right)= & \left(\frac{\delta_{i v}}{2} \tanh t \frac{d}{d t}+\tau\left(Z_{i} \bar{Z}_{v}\right) \tanh ^{2} t\right. \\
& \left.+\frac{\sqrt{-1}}{4} \delta_{i v}\left(1+\tanh ^{2} t\right) \tau\left(Z_{0}\right)\right) \varphi\left(a_{t}\right) \\
R_{X_{i} \bar{X}_{0}} \varphi\left(a_{t}\right)= & \frac{\tau\left(Z_{i}\right)}{2}\left(\tanh t \frac{d}{d t}+\frac{\sqrt{-1}}{2}\left(\tanh ^{2} t+1\right) \tau\left(Z_{0}\right)\right) \varphi\left(a_{t}\right) \\
R_{X_{0} \bar{X}_{i}} \varphi\left(a_{t}\right)= & \frac{\tau\left(\bar{Z}_{i}\right)}{2}\left(\tanh t \frac{d}{d t}+2-\frac{\sqrt{-1}}{2}\left(\tanh ^{2} t+1\right) \tau\left(Z_{0}\right)\right) \varphi\left(a_{t}\right)
\end{aligned}
$$

$$
\begin{aligned}
R_{X_{0} \bar{X}_{0}} \varphi\left(a_{t}\right)=\left(\frac{1}{4} \frac{d^{2}}{d t^{2}}+\frac{1}{4}(\tanh t+\operatorname{coth} t)\right. & \frac{d}{d t}+\frac{\sqrt{-1}}{2} \tau\left(Z_{0}\right) \\
& \left.+\frac{1}{16}(\tanh t+\operatorname{coth} t)^{2} \tau\left(Z_{0}\right)^{2}\right) \varphi\left(a_{t}\right)
\end{aligned}
$$

$$
\begin{aligned}
& R_{X_{i} \bar{X}_{j}} \varphi\left(a_{t}\right)=\tau\left(Z_{i} \bar{Z}_{j}\right) \varphi\left(a_{t}\right) \\
& R_{X_{j} \bar{X}_{i}} \varphi\left(a_{t}\right)=\tau\left(Z_{j} \bar{Z}_{i}\right) \varphi\left(a_{t}\right)
\end{aligned}
$$


(11) $R_{X_{j} \bar{X}_{\mu}} \varphi\left(a_{t}\right)=\left(\frac{\delta_{j \mu}}{2} \operatorname{coth} t \frac{d}{d t}+\tau\left(Z_{j} \bar{Z}_{\mu}\right) \operatorname{coth}^{2} t\right.$

$$
\left.+\frac{\sqrt{-1}}{4}\left(1+\operatorname{coth}^{2} t\right) \delta_{j \mu} \tau\left(Z_{0}\right)\right) \varphi\left(a_{t}\right),
$$

$$
\begin{aligned}
& R_{X_{j} \bar{X}_{0}} \varphi\left(a_{t}\right)=\frac{\tau\left(Z_{j}\right)}{2}\left(\operatorname{coth} t \frac{d}{d t}+\frac{\sqrt{-1}}{2}\left(1+\operatorname{coth}^{2} t\right) \tau\left(Z_{0}\right)\right) \varphi\left(a_{t}\right), \\
& R_{X_{0} \bar{X}_{j}} \varphi\left(a_{t}\right)=\frac{\tau\left(\bar{Z}_{j}\right)}{2}\left(\operatorname{coth} t \frac{d}{d t}+2-\frac{\sqrt{-1}}{2}\left(1+\operatorname{coth}^{2} t\right) \tau\left(Z_{0}\right)\right) \varphi\left(a_{t}\right) .
\end{aligned}
$$

Proof. We prove these formulas through a computation similar to that of [Oda and Tsuzuki 2003, Lemma 7.1.2], using the formulas

$$
\begin{array}{ll}
X_{i}=-\tanh t \cdot Z_{i}+\frac{1}{\cosh t} \operatorname{Ad}\left(a_{t}\right)^{-1} X_{i}, & 1 \leqslant i \leqslant n-r, \\
X_{j}=-\operatorname{coth} t \cdot Z_{j}+\frac{1}{\sinh t} \operatorname{Ad}\left(a_{t}\right)^{-1} Z_{j}, & n-r+1 \leqslant j \leqslant n-1, \\
X_{0}=\frac{1}{2} Y_{0}+\operatorname{coth}(2 t) \frac{\sqrt{-1}}{2} Z_{0}-\frac{\sqrt{-1}}{2 \sinh (2 t)} \operatorname{Ad}\left(a_{t}\right)^{-1} Z_{0}
\end{array}
$$

and their complex conjugates.

Casimir operators. Let $\Omega, \Omega_{K}$ and $\Omega_{M_{0}}$ be the Casimir elements of $\mathfrak{g}, \mathfrak{k}$ and $\mathfrak{m}_{0}:=$ $\operatorname{Lie}\left(M_{0}\right)$ corresponding to the invariant form $\langle X, Y\rangle=2^{-1} \operatorname{tr}(X Y)$. Since $\left\{X_{\alpha}+\bar{X}_{\alpha}\right.$, $\left.\sqrt{-1}\left(X_{\alpha}-\bar{X}_{\alpha}\right)\right\}_{\alpha=0}^{n-1}$ is an orthonormal basis of $\mathfrak{p}$, we have

$$
\Omega=\Omega_{K}+2 \sum_{\alpha=0}^{n-1}\left(X_{\alpha} \bar{X}_{\alpha}+\bar{X}_{\alpha} X_{\alpha}\right)
$$

Since $\left\{Z_{\beta}+\bar{Z}_{\beta}, \sqrt{-1}\left(Z_{\beta}-\bar{Z}_{\beta}\right)\right\}_{\beta=1}^{n-1} \cup\left\{Z_{0}\right\}$ is a pseudo-orthonormal basis of the orthogonal complement of $\mathfrak{m}_{0}$ in $\mathfrak{k}$, we have

$$
\Omega_{K}=\Omega_{M_{0}}-2 \sum_{\beta=1}^{n-1}\left(Z_{\beta} \bar{Z}_{\beta}+\bar{Z}_{\beta} Z_{\beta}\right)-Z_{0}^{2} .
$$

Theorem 2.3. Let $\varphi \in C_{\tau}^{\infty}$ be such that its values $\varphi(g), g \in G$, belong to $\bigwedge^{p, p} \mathfrak{p}_{\mathbb{C}}^{*}$ for $0 \leqslant p \leqslant r$. Put $f(z)=\varphi\left(a_{t}\right)$ where $z=\tanh ^{2} t$. Then $\Omega \varphi\left(a_{t}\right)(t>0)$ equals $4 z(1-z)^{2}\left(\frac{d^{2}}{d z^{2}}+\left(\frac{\mu+1}{z}+\frac{n-1}{1-z}\right) \frac{d}{d z}-\frac{\mathscr{S}}{z(1-z)}+\frac{\mathscr{S}^{\prime}}{z^{2}(1-z)}+\frac{\tau\left(\Omega_{M_{0}}\right)}{4 z(1-z)^{2}}\right) f(z)$, where $\mathscr{S}$ and $\mathscr{S}^{\prime}$ are operators on $\bigwedge \mathfrak{p}_{\mathbb{C}}^{*}$ defined by

$$
\mathscr{Y}:=2^{-1} \sum_{i=1}^{n-r} \tau\left(Z_{i} \bar{Z}_{i}+\bar{Z}_{i} Z_{i}\right) \quad \text { and } \quad \mathscr{S}^{\prime}:=2^{-1} \sum_{j=n-r+1}^{n-1} \tau\left(Z_{j} \bar{Z}_{j}+\bar{Z}_{j} Z_{j}\right)
$$


Proof. By (8) and Proposition 1.2, the operator $\tau\left(Z_{0}\right)$ is zero on the space

$$
\left(\bigwedge^{p, p} \mathfrak{p}_{\mathbb{C}}^{*}\right)^{M}=\left\langle L^{p-j} \mathrm{v}_{0}^{(j)}, L^{p-j} \mathrm{v}_{1}^{(j)} \mid 0 \leqslant j \leqslant p\right\rangle_{\mathbb{C}},
$$

to which the values $\varphi\left(a_{t}\right)(t>0)$ belong by the $(H, K)$-equivariance of $\varphi$. Hence under the assumption, we have $\tau\left(Z_{0}\right) \varphi\left(a_{t}\right)=0$ identically. We also note that the center of $K$ acts on $\bigwedge^{p, p} \mathfrak{p}_{\mathbb{C}}^{*}$ trivially. Noting these remarks and using the fact that $\sum_{\alpha=0}^{n-1}\left[X_{\alpha}, \bar{X}_{\alpha}\right]$ is in the center of $\mathfrak{k}_{\mathbb{C}}$, we obtain the following expression of $\Omega \varphi\left(a_{t}\right)$ from (12) and (13).

$$
\Omega \varphi\left(a_{t}\right)=4 \sum_{\alpha=0}^{n-1} R_{X_{\alpha} \bar{X}_{\alpha}} \varphi\left(a_{t}\right)-2 \sum_{\beta=0}^{n-1} \tau\left(Z_{\beta} \bar{Z}_{\beta}+\bar{Z}_{\beta} Z_{\beta}\right) \varphi\left(a_{t}\right)+\tau\left(\Omega_{M_{0}}\right) \varphi\left(a_{t}\right) .
$$

We can compute the first $n$ terms of the right-hand side of this equation using the formulas (9), (10) and (11) to obtain

$$
\begin{aligned}
\Omega \varphi\left(a_{t}\right)=\left(\frac{d^{2}}{d t^{2}}+\left((2 n-2 r+1) \tanh t+\frac{2 r-1}{\tanh t}\right)\right. & -\frac{4 \mathscr{\varphi}}{\cosh ^{2} t} \frac{d}{d t} \\
& \left.+\frac{4 \varphi^{\prime}}{\sinh ^{2} t}+\tau\left(\Omega_{M_{0}}\right)\right) \varphi\left(a_{t}\right) .
\end{aligned}
$$

The conclusion follows since

$$
\frac{d^{2}}{d t^{2}}=4 z(1-z)^{2} \frac{d^{2}}{d z^{2}}+2(1-z)(1-3 z) \frac{d}{d z}, \quad \frac{d}{d t}=2 z^{1 / 2}(1-z) \frac{d}{d z} .
$$

Lemma 2.4. Let $0<p \leqslant \mu$ and put $l=n-p-r+1$ and $m=r-p$. Then:

$$
\frac{1}{m p} \mathscr{V}_{0}^{(p)}=-\frac{l+m}{m(l+p+m)} \mathscr{S}_{1}^{(p)}=\frac{-1}{l+m} \mathrm{v}_{0}^{(p)}+\frac{l}{m} \mathrm{v}_{1}^{(p)}
$$

$$
\begin{aligned}
-\frac{1}{l p} \mathscr{S}^{\prime} \mathrm{v}_{0}^{(p)} & =-\frac{l+m}{m(l+p+m)} \mathscr{S}^{\prime} \mathrm{v}_{1}^{(p)}=\frac{1}{l+m} \mathrm{v}_{0}^{(p)}+\mathrm{v}_{1}^{(p)} \\
\tau\left(\Omega_{M_{0}}\right) \mathrm{v}_{0}^{(p)} & =4 p(n-p) \mathrm{v}_{0}^{(p)} \text { and } \tau\left(\Omega_{M_{0}}\right) \mathrm{v}_{1}^{(p)}=4(p-1)(n-p+1) \mathrm{v}_{1}^{(p)} .
\end{aligned}
$$

Proof. As shown in the proof of Proposition 1.2, the tensors $v_{0}^{(p)}$ and $v_{1}^{(p)}$ are obtained as the image of $\mathrm{u}^{(p)} \in E_{p, p}$ and $\mathrm{u}^{(p-1)} \in E_{p-1, p-1}$ respectively by the $M_{0^{-}}$ homomorphism $\mathscr{T}^{p, p}$. Hence in order to prove (18) we have only to check that the eigenvalue of $\Omega_{M_{0}}$ on $E_{p, p}$ equals $4 p(n-p)$. The eigenvalue is easily calculated if we note that the highest weight of an irreducible $M_{0}=(\mathrm{U}(n-1) \times \mathrm{U}(1))$-module $E_{p, p}$ is

$$
(\overbrace{1, \ldots, 1}^{p}, \overbrace{0, \ldots, 0}^{n-2 p-1}, \overbrace{-1 \ldots,-1}^{p} ; 0) .
$$

We prove the formula (17). Since $\mathrm{w}^{(p)}$ is $(H \cap K)$-invariant and since the elements $\bar{Z}_{j}, Z_{j}(n-r+1 \leqslant j \leqslant n-1)$ belong to $(\mathfrak{h} \cap \mathfrak{k})_{\mathbb{C}}$, we have $\mathscr{Y}^{\prime} \mathbf{w}^{(p)}=0$. This 
together with (5) gives the first equality of (17). To obtain the second equality we need some computation. From definition we have the expressions

$$
\begin{aligned}
& \mathrm{v}_{1}^{(p)}=\frac{-1}{n-2 p+1} c_{p}^{(p)} \frac{\sqrt{-1}}{2}(r-p) \omega_{0} \wedge \bar{\omega}_{0} \wedge \eta^{p-1}+L\left(\xi_{1}\right) \\
& \mathrm{v}_{0}^{(p)}=\frac{1}{n-p+1} c_{p}^{(p)}\left((n-2 p+1) \eta^{p}+\frac{\sqrt{-1}}{2} p(r-p) \omega_{0} \wedge \bar{\omega}_{0} \wedge \eta^{p-1}\right)+L\left(\xi_{0}\right),
\end{aligned}
$$

for some $\xi_{0}$ and $\xi_{1}$. Using Lemma 2.1 and (2), we compute that

$$
\begin{aligned}
\tau\left(\bar{Z}_{j} Z_{j}\right)\left(\omega_{0} \wedge \bar{\omega}_{0} \wedge \eta^{p-1}\right)=\omega_{j} \wedge \bar{\omega}_{j} \wedge \eta^{p-1}+\omega_{0} \wedge\left(-\bar{\omega}_{0}\right) \wedge \eta^{p-1} \\
\quad-\frac{\sqrt{-1}}{2}(p-1) \omega_{0} \wedge \bar{\omega}_{j} \wedge \omega_{j} \wedge \bar{\omega}_{0} \wedge \eta^{p-2}
\end{aligned}
$$

for $n-r+1 \leqslant j \leqslant n-1$. Taking the sum over $j$, we obtain

$$
\sum_{j=n-r+1}^{n-1} \tau\left(\bar{Z}_{j} Z_{j}\right)\left(\omega_{0} \wedge \bar{\omega}_{0} \wedge \eta^{p-1}\right)=-(r-p) \omega_{0} \wedge \bar{\omega}_{0} \wedge \eta^{p-1}+\frac{2}{\sqrt{-1}} \eta^{p} .
$$

We use (19) to write the right-hand side in terms of $\mathrm{v}_{0}^{(p)}$ and $\mathrm{v}_{1}^{(p)}$ :

$$
\begin{aligned}
\sum_{j=n-r+1}^{n-1} \tau\left(\bar{Z}_{j} Z_{j}\right)\left(\omega_{0} \wedge \bar{\omega}_{0} \wedge\right. & \left.\eta^{p-1}\right) \\
& =\frac{2}{\sqrt{-1}} \frac{n-p+1}{c_{p}^{(p)}}\left(\frac{1}{n-2 p+1} \mathrm{v}_{0}^{(p)}+\mathrm{v}_{1}^{(p)}\right)+L\left(\xi_{2}\right)
\end{aligned}
$$

for some $\xi_{2}$. From this and the first equality in (19), we obtain

$$
\sum_{j=n-r+1}^{n-1} \tau\left(\bar{Z}_{j} Z_{j}\right) \mathrm{v}_{1}^{(p)}=-\frac{(r-p)(n-p+1)}{n-2 p+1}\left(\frac{1}{n-2 p+1} \mathrm{v}_{0}^{(p)}+\mathrm{v}_{1}^{(p)}\right)+L\left(\xi_{3}\right)
$$

for some $\xi_{3}$. Since all terms in this identity except $L\left(\xi_{3}\right)$ belong to $F_{p, p}$ and since $F_{p, p} \cap \operatorname{Im} L=\{0\}$, the residual term $L\left(\xi_{3}\right)$ has to be zero. Noting that $\mathrm{v}_{0}^{(p)}$ and $\mathrm{v}_{1}^{(p)}$ are real elements of $\wedge \mathfrak{p}_{\mathbb{C}}^{*}$, we know that $2 \mathscr{S}^{\prime} \mathrm{v}_{1}^{(p)}$ is given by the sum of $(20)$ and its complex conjugate. This completes the proof of the second identity of (17). We can deduce (16) from (17) and (18) using the relation

$$
\tau\left(\Omega_{K}\right) v_{i}^{(p)}=\tau\left(\Omega_{M_{0}}\right) v_{i}^{(p)}-4 \mathscr{S} v_{i}^{(p)}-4 \mathscr{S}^{\prime} v_{i}^{(p)}
$$

obtained from (13), since the eigenvalue of $\Omega_{K}$ on $F_{p, p}$ equals $4 p(n+1-p)$.

Let $0<p \leqslant \mu$ and $\varphi \in C_{\tau}^{\infty}$ be such that its values $\varphi(g)$ belong to $F_{p, p}$. By the $(H, K)$-equivariance, the vector $\varphi\left(a_{t}\right)$ belongs to the space of $M$-fixed tensors $F_{p, p}^{M}=\mathbb{C} v_{0}^{(p)} \oplus \mathbb{C} v_{1}^{(p)}$. We can write

$$
\varphi\left(a_{t}\right)=f_{0}(z) \mathrm{v}_{0}^{(p)}+f_{1}(z) \mathrm{v}_{1}^{(p)}=\left[\mathrm{v}_{0}^{(p)} \mathrm{v}_{1}^{(p)}\right] \mathrm{F}(z), \quad z=\tanh ^{2} t \in(0,1),
$$


with a $\mathbb{C}^{2}$-valued $C^{\infty}$-function

$$
\mathrm{F}(z)=\left[\begin{array}{l}
f_{0}(z) \\
f_{1}(z)
\end{array}\right]
$$

on $0<z<1$. Given a complex number $s$, consider the differential equation

$$
\Omega \varphi(g)=\left(s^{2}-\lambda^{2}\right) \varphi(g), \quad g \in G-H K .
$$

We rewrite this equation in terms of $F(z)$.

Proposition 2.5. Let $\mathrm{Q}^{(p)}(z):=\left[\begin{array}{ll}Q_{0}^{0}(z) & Q_{0}^{1}(z) \\ Q_{1}^{0}(z) & Q_{1}^{1}(z)\end{array}\right]$ with

$Q_{0}^{0}(z)=\frac{1}{z^{2}(1-z)^{2}}\left(-\frac{p m}{l+m} z^{2}+\left(p-(m-1) l+\frac{1}{4}\left(n^{2}-s^{2}\right)\right) z-\frac{l p}{l+m}\right)$,

$Q_{0}^{1}(z)=-\frac{m(l+p+m)}{(l+m)^{2}} \frac{1+z}{z^{2}(1-z)}$,

$Q_{1}^{0}(z)=-l p \frac{1+z}{z^{2}(1-z)}$,

$Q_{1}^{1}(z)=\frac{1}{z^{2}(1-z)^{2}}\left(-\frac{l(l+p+m)}{l+m} z^{2}+\left(p-(m-1) l+\frac{1}{4}\left(n^{2}-s^{2}\right)\right) z\right.$

$$
\left.-\frac{m(l+p+m)}{l+m}\right)
$$

Then $\varphi \in C_{\tau}^{\infty}$ with values in $F_{p, p}$ is a solution of (22) if and only if $\mathrm{F}(z)(0<z<1)$ satisfies the second order ordinary differential equation

$$
\frac{d^{2} \mathrm{~F}}{d z^{2}}+\left(\frac{\mu+1}{z}+\frac{n-1}{1-z}\right) \frac{d \mathrm{~F}}{d z}+\mathrm{Q}^{(p)}(z) \mathrm{F}=0 .
$$

Proof. This follows from Theorem 2.3 and Lemma 2.4 .

The Schmid operator. Let $\nabla_{ \pm}$be the Schmid operator, which is given by

$$
\nabla_{+} f(g)=\sum_{i=0}^{n-1} e\left(\bar{\omega}_{i}\right) R_{\bar{X}_{i}} f(g) \quad \text { and } \quad \nabla_{-} f(g)=\sum_{i=0}^{n-1} e\left(\omega_{i}\right) R_{X_{i}} f(g)
$$

for a $\wedge \mathfrak{p}_{\mathbb{C}}^{*}$-valued $C^{\infty}$-function $f$ on an open subset of $G$. To describe the radial part of the Schmid operator, we introduce four operators acting on $\wedge \mathfrak{p}_{\mathbb{C}}^{*}$ : 


$$
\begin{array}{ll}
\mathscr{P}_{+}=\sum_{i=1}^{n-r} e\left(\bar{\omega}_{i}\right) \tau\left(\bar{Z}_{i}\right), & \mathscr{P}_{-}=\sum_{i=1}^{n-r} e\left(\omega_{i}\right) \tau\left(Z_{i}\right), \\
\mathscr{R}_{+}=\sum_{j=n-r+1}^{n-1} e\left(\bar{\omega}_{j}\right) \tau\left(\bar{Z}_{j}\right), & \mathscr{R}_{-}=\sum_{j=n-r+1}^{n-1} e\left(\omega_{j}\right) \tau\left(Z_{j}\right) .
\end{array}
$$

Theorem 2.6. Let $\varphi \in C_{\tau}^{\infty}$ and $f(z)$ be the same as in Theorem 2.3. Then for $t>0$, $\nabla_{-} \nabla_{+} \varphi\left(a_{t}\right)$ equals

$$
\begin{aligned}
z(1-z)^{2}\left(e\left(\omega_{0} \wedge \bar{\omega}_{0}\right) \frac{d^{2}}{d z^{2}}-\left(\frac{\mathscr{A}}{z}\right.\right. & \left.+\frac{\mathscr{B}}{1-z}\right) \frac{d}{d z} \\
& \left.+\frac{\mathscr{P}_{-} \mathscr{P}_{+}}{(1-z)^{2}}+\frac{\mathscr{R}_{-} \mathscr{R}_{+}}{z^{2}(1-z)^{2}}+\frac{\mathscr{C}}{z(1-z)^{2}}\right) z f(z)
\end{aligned}
$$

where the numerators $\mathscr{A}, \mathscr{B}$ and $\mathscr{C}$ are given by

$$
\begin{aligned}
& \mathscr{A}=-\frac{2}{\sqrt{-1}} e\left(\omega-\omega_{H}\right)+e\left(\bar{\omega}_{0}\right) \mathscr{R}_{-}-e\left(\omega_{0}\right) \mathscr{R}_{+}, \\
& \mathscr{P}=-\frac{\sqrt{-1}}{2} e(\omega)+e\left(\omega_{0} \wedge \bar{\omega}_{0}\right)+e\left(\bar{\omega}_{0}\right)\left(\mathscr{P}_{-}+\mathscr{R}_{-}\right)-e\left(\omega_{0}\right)\left(\mathscr{P}_{+}+\mathscr{R}_{+}\right), \\
& \mathscr{C}=e\left(\omega_{0}\right)\left(\mathscr{P}_{+}+\mathscr{R}_{+}\right)+\mathscr{P}_{-} \mathscr{R}_{+}+\mathscr{R}_{-} \mathscr{P}_{+} .
\end{aligned}
$$

Proof. Input the formulas in Lemma 2.2 to the right-hand side of the identity

$$
\nabla_{-} \nabla_{+} \varphi\left(a_{t}\right)=\sum_{\alpha, \beta=0}^{n-1} R_{X_{\alpha} \bar{X}_{\beta}} \varphi\left(a_{t}\right) \wedge \omega_{\alpha} \wedge \bar{\omega}_{\beta} .
$$

Then a direct computation using (15) yields (24).

\section{Secondary spherical functions}

Before we state the main theorem of this section, we prove a lemma which is important not only in this section but also in the global theory to be developed in Section 5.

Lemma 3.1. For each integer $p$ with $1 \leqslant p \leqslant r$, there exists a unique holomorphic function $s \mapsto v_{s}^{(p)}$ on the domain $\mathbb{C}-L_{p}$ with

$$
L_{p}=\{s \in \sqrt{-1} \mathbb{R}|2 \sqrt{(r-p)(n-p-r+2)} \geqslant| \operatorname{Im} s \mid\},
$$

which takes a positive real value for $s>0$ and such that

$$
\left\{v_{s}^{(p)}\right\}^{2}=s^{2}+4(r-p)(n-p-r+2) .
$$

The functional equation $v_{-s}^{(p)}=-v_{s}^{(p)}$ holds for $s \in \mathbb{C}-L_{p}$. Also, if $\operatorname{Re} s>0$, then $\operatorname{Re} v_{s}^{(p)}>\operatorname{Re} v_{s}^{(p+1)}>\operatorname{Re} s$. 
Proof. Put $c_{p}=4(r-p)(n-p-r+2)$. By the residue theorem, the value (mod $2 \pi \sqrt{-1} \mathbb{Z})$ of the integral

$$
I(s):=\int_{C_{s}} \frac{\zeta}{\zeta^{2}+c_{p}} d \zeta
$$

is independent of the choice of the path $C_{s}$ connecting 1 and $s$ inside the region $\mathbb{C}-L_{p}$, and locally defines a holomorphic function on $\mathbb{C}-L_{p}$. Since $v_{s}^{(p)}=$ $\exp (I(s)) \sqrt{c_{p}+1}$ for $s \in \mathbb{C}-L_{p}$, the first assertion follows. Put $\sigma=\operatorname{Re} s$ and $t=\operatorname{Im} s$ and suppose $\sigma>0$. Then we have

$$
\operatorname{Re} v_{s}^{(p)}=2^{-1 / 2}\left(x_{p, s}+\sqrt{x_{p, s}^{2}+y_{s}^{2}}\right)^{1 / 2},
$$

with $x_{p, s}=\sigma^{2}-t^{2}+c_{p}, y_{s}=2 \sigma t$. Since $c_{p}>c_{p+1}$ and $x_{p, s}>x_{p+1, s}$, we have $\operatorname{Re} v_{s}^{(p)}>\operatorname{Re} v_{s}^{(p+1)}$. The formula $\operatorname{Re} v_{s}^{(p)}=\left(\sigma^{2}+2^{-1}\left(\sqrt{T^{2}+4 c_{p} \sigma^{2}}-T\right)\right)^{1 / 2}$ with $T=t^{2}+\sigma^{2}-c_{p}$ obviously implies the inequality $\operatorname{Re} v_{s}^{(p)}>\sigma$.

To prove $v_{-s}^{(p)}=-v_{s}^{(p)}$, take a path $C_{s,-s}$ lying in the domain $\mathbb{C}-L_{p}$ from $s$ to $-s$ and $C^{\prime}$ the image of $C_{s,-s}$ by the map $z=\zeta^{2}+c_{p}$. Then $C^{\prime}$ is a simple loop rounding the point $\zeta=0$. By definition, $v_{s}^{(p)}=\exp (I(s)-I(-s)) \nu_{-s}^{(p)}$. Since

$$
I(s)-I(-s)=\int_{C_{s,-s}} \frac{\zeta}{\zeta^{2}+c_{p}} d \zeta=\frac{1}{2} \int_{C^{\prime}} \frac{d z}{z}= \pm \pi \sqrt{-1},
$$

we have $v_{-s}^{(p)}=-v_{s}^{(p)}$.

Consider the holomorphic function

$$
d(s):=\prod_{p=1}^{r} \Gamma\left(v_{s}^{(p)}\right)^{-1} \Gamma\left(2^{-1}\left(v_{s}^{(p)}-\lambda\right)+1\right)^{-1}, \quad s \in \mathbb{C}-L_{1},
$$

and define the related sets

$$
D=\left\{s \in \mathbb{C}-L_{1} \mid d(s) \neq 0\right\}, \quad \tilde{D}=\bigcap_{p=1}^{\mu}\left\{s \in D \mid \operatorname{Re} v_{s}^{(p)}+\operatorname{Re} v_{s}^{(p+1)}>4\right\} .
$$

Note that $\{s \in \mathbb{C} \mid \operatorname{Re} s>\lambda\} \subset \tilde{D}$.

The aim of this section is to prove the following theorem.

Theorem 3.2. There is a unique family of $C^{\infty}$-functions $\phi_{s}: G-H K \rightarrow \wedge^{\mu, \mu} \mathfrak{p}_{\mathbb{C}}^{*}, s$ varying over $\tilde{D}$, satisfying the following conditions.

(i) For each $g \in G-H K$, the function $s \mapsto \phi_{s}(g)$ is holomorphic.

(ii) $\phi_{s}$ has the $(H, K)$-equivariance property

$$
\phi_{s}(h g k)=\tau(k)^{-1} \phi_{s}(g), \quad h \in H, k \in K, g \in G-H K .
$$


(iii) $\phi_{s}$ satisfies the differential equation

$$
\Omega \phi_{s}(g)=\left(s^{2}-\lambda^{2}\right) \phi_{s}(g), \quad g \in G-H K .
$$

(iv) (Recall the $(H \cap K)$-invariant tensor $w \in \bigwedge^{\mu, \mu} \mathfrak{p}_{\mathbb{C}}^{*}$ defined in Proposition 1.4.) We have

$$
\lim _{t \rightarrow+0} t^{2 \mu} \phi_{s}\left(a_{t}\right)=\mathrm{w} .
$$

(v) If $\operatorname{Re} s>n$, then $\phi_{s}\left(a_{t}\right)$ decays exponentially as $t \rightarrow+\infty$.

We call the function $\phi_{s}$ the secondary spherical function.

Differential equations. In this subsection, we fix an $s \in D$. Lemma 1.1 yields the decomposition

$$
\bigwedge^{\mu, \mu} \mathfrak{p}_{\mathbb{C}}^{*}=\sum_{p=0}^{\mu} L^{\mu-p}\left(F_{p, p}\right)
$$

of $K$-modules. Hence in order to obtain the function $\phi_{s}$ as in Theorem 3.2, we have only to construct a function $\phi_{s}^{(p)}: G-H K \rightarrow F_{p, p}$ for each $0 \leqslant p \leqslant \mu$ with the same properties as $\phi_{s}$ listed in Theorem 3.2 except condition (iv). Instead we require

$$
\lim _{t \rightarrow+0} t^{2 \mu} \phi_{s}^{(p)}\left(a_{t}\right)=\mathrm{w}^{(p)},
$$

and then form $\phi_{s}(g)=\sum_{p=0}^{\mu} \gamma_{p} L^{\mu-p}\left(\phi_{s}^{(p)}(g)\right)$.

From now on we fix $0 \leqslant p \leqslant \mu$ and examine the conditions to be satisfied by $\phi_{s}^{(p)}$. We use the notation $l=n-p-r+1, m=r-p$ as in Lemma 2.4.

The case $p>0$. Since $\phi_{s}^{(p)}$ should be a solution of (22), we first analyze the differential equation (23) in some detail.

Proposition 3.3. Consider a $\mathbb{C}^{2}$-valued $C^{\infty}$-function $\mathrm{F}(z)=\left[\begin{array}{l}f_{0}(z) \\ f_{1}(z)\end{array}\right], 0<z<1$. The following conditions on $\mathrm{F}(z)$ are equivalent.

(a) $\mathrm{F}(z)$ is a solution of (23) and also satisfies

$$
f_{0}(z)=-\frac{z}{l p}\left(\frac{d}{d z}+\frac{m(l+p+m)}{l+m} \frac{1}{z}+\frac{l+p+m}{1-z}\right) f_{1}(z) .
$$

(b) $\mathrm{F}(z)$ is a solution of

$$
\frac{d \mathrm{~F}}{d z}=\mathrm{B}^{(p)}(z) \mathrm{F}
$$


where $\mathrm{B}^{(p)}(z)=\left[\begin{array}{ll}B_{0}^{0}(z) & B_{0}^{1}(z) \\ B_{1}^{0}(z) & B_{1}^{1}(z)\end{array}\right]$ with entries

$$
\begin{aligned}
& B_{0}^{0}(z)=-\frac{l p}{l+m} \frac{1}{z}-\frac{p}{1-z}, \quad B_{1}^{0}(z)=-\frac{l p}{z}, \\
& B_{0}^{1}(z)=-\frac{m(l+p+m)}{(l+m)^{2}} \frac{1}{z}-\frac{s^{2}-(l-m+1)^{2}}{4 l p} \frac{1}{(1-z)^{2}}, \\
& B_{1}^{1}(z)=-\frac{m(l+p+m)}{l+m} \frac{1}{z}-\frac{l+p+m}{1-z} .
\end{aligned}
$$

(c) The entries $f_{0}$ and $f_{1}$ of $\mathrm{F}(z)$ satisfy (28) and

$$
\begin{aligned}
\left(\frac{d^{2}}{d z^{2}}+\left(\frac{\mu+2}{z}+\frac{n+1}{1-z}\right) \frac{d}{d z}\right. & \\
& \left.+\frac{(n+2)^{2}-4 l(m-1)-s^{2}}{4}\left(\frac{1}{z}+\frac{1}{1-z}+\frac{1}{(1-z)^{2}}\right)\right) f_{1}(z)=0 .
\end{aligned}
$$

Proof. This follows by direct computation.

Remark. When $\mathrm{F}(z)$ is related to some $\varphi \in C_{\tau}^{\infty}$ by the relation (21), the equation (28) comes from $\operatorname{Pr}\left(\nabla_{+} \varphi\left(a_{t}\right)\right)=0$, where $\operatorname{Pr}: F_{p, p} \otimes \mathfrak{p}_{-}^{*} \rightarrow F_{p, p-1}$ is a certain $K$-projector.

Lemma 3.4. Let $\mathrm{F}_{0}(z)$ and $\mathrm{F}_{1}(z)$ be two solutions of $(29)$ and form the $2 \times 2$-matrix valued function $\Phi(z):=\left[\mathrm{F}_{0}(z) \mathrm{F}_{1}(z)\right]$. Consider a $\mathbb{C}^{2}$-valued $C^{\infty}$-function $\mathrm{A}(z)$ on $0<z<1$. Then the following two conditions on $\mathrm{A}(z)$ are equivalent.

(a) The function $\tilde{\mathrm{F}}(z)=\Phi(z) \mathrm{A}(z)$ is a solution of the differential equation (23).

(b) The function $\mathrm{U}(z)=\Phi(z) \frac{d \mathrm{~A}}{d z}(z)$ satisfies the differential equation

$$
\frac{d \mathrm{U}}{d z}=-\left(\mathrm{B}^{(p)}(z)+\left(\frac{\mu+1}{z}+\frac{n-1}{1-z}\right)\left[\begin{array}{ll}
1 & 0 \\
0 & 1
\end{array}\right]\right) \mathrm{U} .
$$

Proof. Put $p(z)=(\mu+1) z^{-1}+(n-1)(1-z)^{-1}$. Since $\Phi(z)$ satisfies

$$
\frac{d^{2} \Phi}{d z^{2}}+p(z) \frac{d \Phi}{d z}+\mathrm{Q}^{(p)}(z) \Phi=0 \quad \text { and } \quad \frac{d \Phi}{d z}=\mathrm{B}^{(p)}(z) \Phi,
$$

we have

$$
\begin{aligned}
\left(\frac{d^{2}}{d z^{2}}+p(z) \frac{d}{d z}+\mathrm{Q}^{(p)}(z)\right)( & (z) \mathrm{A}(z)) \\
= & \left(\frac{d}{d z}+\left(\mathrm{B}^{(p)}(z)+p(z)\left[\begin{array}{ll}
1 & 0 \\
0 & 1
\end{array}\right]\right)\right)\left(\Phi(z) \frac{d \mathrm{~A}(z)}{d z}\right),
\end{aligned}
$$

by a direct computation. This identity proves the equivalence of (a) and (b). 
Lemma 3.5. Consider a $\mathbb{C}^{2}$-valued $C^{\infty}$-function $\mathrm{U}(z)=\left[\begin{array}{l}u_{0}(z) \\ u_{1}(z)\end{array}\right]$ on $0<z<1$. Then $\mathrm{U}$ is a solution of (31) if and only if it satisfies

$$
\frac{z}{l p}\left(\frac{d}{d z}+\frac{l p}{l+m} \frac{1}{z}+\frac{p-2}{1-z}\right) u_{1}(z)=u_{0}(z)
$$

and

$$
\begin{aligned}
\left(\frac{d^{2}}{d z^{2}}+\left(\frac{\mu+2}{z}+\frac{n-3}{1-z}\right) \frac{d}{d z}\right. & +\frac{n^{2}-s^{2}-4 l(m-1)-8(m+1)}{4}\left(\frac{1}{z}+\frac{1}{1-z}\right) \\
& \left.+\frac{n^{2}-s^{2}-4 l(m-1)-8(n-p)}{4} \frac{1}{(1-z)^{2}}\right) u_{1}(z)=0 .
\end{aligned}
$$

Proof. This is a direct computation.

The case $p=0$. Since the function $\phi_{s}^{(0)}$ is $F_{0,0}=\mathbb{C}$-valued, we can write, thanks to $(H, K)$-equivariance,

$$
\phi_{s}^{(0)}\left(a_{t}\right)=f_{0}(z), \quad z=\tanh ^{2} t,
$$

with a $C^{\infty}$-function $f_{0}(z)$ on $0<z<1$. From (22) we obtain:

Proposition 3.6. The function $f_{0}(z)$ satisfies the differential equation

$$
\left(\frac{d^{2}}{d z^{2}}+\left(\frac{\mu+1}{z}+\frac{n-1}{1-z}\right) \frac{d}{d z}+\frac{1}{z(1-z)^{2}} \frac{\lambda^{2}-s^{2}}{4}\right) f_{0}(z)=0 .
$$

Construction of solutions. Let $N$ be a nonnegative integer and $\gamma_{i}(0 \leqslant i \leqslant N)$ complex numbers. For a function $f(s, z)$ on $\{(s, z) \mid s \in D, 0<z<1\}$, if there exist a meromorphic function $q(s)$ on $\mathbb{C}-L_{1}$ holomorphic on $D$ and a family of holomorphic functions $\alpha(s), a_{i}(s ; z)(0 \leqslant i \leqslant N)$ on $\left\{(s, z)\left|s \in \mathbb{C}-L_{1},\right| z \mid<\varepsilon\right\}$ with some $\varepsilon>0$ such that

$$
f(s ; z)=q(s) z^{\alpha(s)}\left(1+\sum_{i=0}^{N} z^{\gamma_{i}}(\log z)^{i} a_{i}(s ; z)\right), \quad 0<z<\varepsilon, s \in D,
$$

then we write $f(s ; z)=q(s) z^{\alpha(s)}\left(1+O\left(s ; z^{\gamma_{0}}, z^{\gamma_{1}} \log z, \ldots, z^{\gamma_{N}}(\log z)^{N}\right)\right)$. Given a $\mathbb{C}^{2}$-valued function

$$
\mathrm{F}(s ; z)=\left[\begin{array}{l}
f_{0}(s ; z) \\
f_{1}(s ; z)
\end{array}\right]
$$

on $\{(s, z) \mid s \in D, 0<z<1\}$, we write

$$
\mathrm{F}(s ; z)=\left[\begin{array}{l}
q_{0}(s) z^{\alpha_{0}(s)} \\
q_{1}(s) z^{\alpha_{1}(s)}
\end{array}\right]\left(1+O\left(s ; z^{\gamma_{0}}, z^{\gamma_{1}} \log z, \ldots, z^{\gamma_{N}}(\log z)^{N}\right)\right)
$$

when $f_{j}(s ; z)=q_{j}(s) y^{\alpha_{j}(s)}\left(1+O\left(s ; z^{\gamma_{0}}, z^{\gamma_{1}} \log z, \ldots, z^{\gamma_{N}}(\log z)^{N}\right)\right)$ for $j=0,1$. 
We define three new functions:

$$
\begin{aligned}
c(s) & :=\frac{\Gamma(s+1) \Gamma(\mu+2)}{\Gamma((s+n) / 2+1) \Gamma((s-\lambda) / 2+1)}, \\
h_{s}(z) & :={ }_{2} F_{1}\left(-\frac{s-n}{2}+1,-\frac{s+\lambda}{2}+1 ; \mu+2 ; z\right), \\
H_{s}(z) & :={ }_{2} F_{1}\left(\frac{s-n}{2}, \frac{s+\lambda}{2} ; s+1 ; 1-z\right) .
\end{aligned}
$$

Note that $c(s)$ has no poles nor zeros in the domain $D$.

Lemma 3.7. Let $s \in D$. Then

$$
h_{s}(z)=1+O(s ; z) \quad \text { and } \quad H_{s}(z)=\frac{c(s)}{\mu+1}\left(1+O\left(s ; z, z^{\mu+1} \log z\right)\right) .
$$

With $y=1-z$, we have

$$
h_{s}(z)=\frac{c(s)}{s}(1+O(s ; y)) \text { if } \operatorname{Re} s>0, \quad \text { and } \quad H_{s}(z)=1+O(s ; y) .
$$

Proof. Use [Magnus et al. 1966, p. 49, line 6 and p. 47, last line].

The case $p>0$.

Proposition 3.8. For $i=0,1$, define $\mathrm{F}_{i}^{(p)}(s ; z)=\left[\begin{array}{l}f_{0 i}^{(p)}(s ; z) \\ f_{1 i}^{(p)}(s ; z)\end{array}\right], 0<z<1, s \in D$,

$$
\begin{aligned}
f_{10}^{(p)}(s ; z) & =(1-z)^{\left(-v_{s}^{(p+1)}+n\right) / 2+1} h_{v_{s}^{(p+1)}}(z), \\
f_{11}^{(p)}(s ; z) & =z^{-(\mu+1)}(1-z)^{\left(v_{s}^{(p+1)}+n\right) / 2+1} H_{v_{s}^{(p+1)}}(z), \\
f_{00}^{(p)}(s ; z) & =-\frac{(1-z)^{\left(-v_{s}^{(p+1)}+n\right) / 2}}{l p}\left(z(1-z) \frac{d}{d z}+\frac{v_{s}^{(p+1)}+l+m-1}{2} z\right. \\
& \left.+\frac{m(l+p+m)}{l+m}(1-z)\right) h_{v_{s}^{(p+1)}}(z), \\
f_{01}^{(p)}(s ; z) & =-\frac{z^{-(\mu+1)}(1-z)^{\left(v_{s}^{(p+1)}+n\right) / 2}}{l p}\left(z(1-z) \frac{d}{d z}+\frac{-v_{s}^{(p+1)}+l+m-1}{2} z\right. \\
& \left.-\frac{l p}{l+m}(1-z)\right) H_{v_{s}^{(p+1)}}(z) .
\end{aligned}
$$

Then $\mathrm{F}_{i}^{(p)}(s ; z)(i=0,1)$ is a $C^{\infty}$-solution of $(29)$ such that

$$
\begin{aligned}
& \mathrm{F}_{0}^{(p)}(s ; z)=\left[\begin{array}{c}
-m(l+p+m) /(l p(l+m)) \\
1
\end{array}\right](1+O(s ; z)), \\
& \mathrm{F}_{1}^{(p)}(s ; z)=\frac{c\left(v_{s}^{(p+1)}\right)}{\mu+1}\left[\begin{array}{c}
1 /(l+m) \\
1
\end{array}\right] z^{-(\mu+1)}\left(1+O\left(s ; z, z^{\mu+1} \log z\right)\right),
\end{aligned}
$$


and such that for $\operatorname{Re} s>0$ and $y=1-z$,

$$
\begin{aligned}
& \mathrm{F}_{0}^{(p)}(s ; z)=\frac{c\left(v_{s}^{(p+1)}\right)}{v_{s}^{(p+1)}}\left[\begin{array}{c}
-\left(v_{s}^{(p+1)}+l+m-1\right) /(2 l p) \\
y
\end{array}\right] y^{\left(-v_{s}^{(p+1)}+n\right) / 2}(1+O(s ; y)), \\
& \mathrm{F}_{1}^{(p)}(s ; z)=\left[\begin{array}{c}
-\left(-v_{s}^{(p+1)}+l+m-1\right) /(2 l p) \\
y
\end{array}\right] y^{\left(v_{s}^{(p+1)}+n\right) / 2}(1+O(s ; y)),
\end{aligned}
$$

Proof. By Proposition 3.3, we have only to check that the functions $f_{0 i}^{(p)}(s ; z)$ and $f_{1 i}^{(p)}(s ; z)$ are solutions of the equations (28) and (30) for each $i=0,1$. This is done by a direct computation. Using Lemma 3.7, we obtain (33) and (34).

Remark. The function $\mathrm{F}_{0}^{(p)}\left(\sqrt{s^{2}-4 l(m-1)} ; \tanh ^{2} t\right)$ is the $A$-radial part of an Eisenstein integral for $H \backslash G$ associated with the principal series $\operatorname{Ind}_{P_{0}}^{G}\left(E_{p, p} ; s\right)$, to be defined in the proof of Proposition 5.5.

By Propositions 3.3 and $3.8, \mathrm{~F}_{0}^{(p)}(s ; z)$ and $\mathrm{F}_{1}^{(p)}(s ; z)$ are two linearly independent solutions of (23). We proceed to construct two more solutions using Lemma 3.4 .

Proposition 3.9. For $i=0,1$, define the function $\mathrm{U}_{i}^{(p)}(s ; z)=\left[\begin{array}{l}u_{0 i}^{(p)}(s ; z) \\ u_{1 i}^{(p)}(s ; z)\end{array}\right], s \in D$,
$0<z<1$, by

$$
\begin{aligned}
& \begin{aligned}
u_{10}^{(p)}(s ; z) & =(1-z)^{\left(-v_{s}^{(p)}+n\right) / 2-1} h_{v_{s}^{(p)}}(z), \\
u_{11}^{(p)}(s ; z)= & z^{-(\mu+1)}(1-z)^{\left(v_{s}^{(p)}+n\right) / 2-1} H_{v_{s}^{(p)}}(z), \\
u_{00}^{(p)}(s ; z)=\frac{(1-z)^{\left(-v_{s}^{(p)}+n\right) / 2-2}}{l p}\left(z(1-z) \frac{d}{d z}+\frac{v_{s}^{(p)}-l-(m+1)}{2} z\right. & \left.\quad+\frac{l p}{l+m}(1-z)\right) h_{v_{s}^{(p)}}(z), \\
u_{01}^{(p)}(s ; z)=\frac{z^{-(\mu+1)}(1-z)^{\left(v_{s}^{(p)}+n\right) / 2-2}}{l p}\left(z(1-z) \frac{d}{d z}+\frac{-v_{s}^{(p)}-l-(m+1)}{2} z\right. & \left.-\frac{m(l+p+m)}{l+m}(1-z)\right) H_{v_{s}^{(p)}}(z) .
\end{aligned}
\end{aligned}
$$

Then $\mathrm{U}_{i}^{(p)}(s ; z)$ is a $C^{\infty}$-solution of $(31)$.

Proof. A direct computation shows $u_{0 i}^{(p)}(s z)$ and $u_{1 i}^{(p)}(s ; z)$ satisfy the two differential equations of Lemma 3.5. The conclusion then follows from Lemma 3.5.

Lemma 3.10. Let $\mathrm{F}_{i}^{(p)}(s ; z)$ be the solutions defined in Proposition 3.8 and form the matrix-valued function $\Phi^{(p)}(s ; z)=\left[\mathrm{F}_{0}^{(p)}(s ; z) \mathrm{F}_{1}^{(p)}(s ; z)\right](0<z<1)$. Define 
the function

$$
\mathrm{A}_{i}^{(p)}(s ; z)=\left[\begin{array}{c}
a_{0 i}^{(p)}(s ; z) \\
a_{1 i}^{(p)}(s ; z)
\end{array}\right], \quad 0<z<1, s \in \tilde{D},
$$

for $i=0,1, b y$

$$
\begin{aligned}
& a_{00}^{(p)}(s ; z)=-z(1-z)^{\left(\nu_{s}^{(p+1)}-v_{s}^{(p)}\right) / 2-1} H_{v_{s}^{(p+1)}}(z) h_{v_{s}^{(p)}}(z) \\
& +\int_{0}^{z}(1-w)^{\left(v_{s}^{(p+1)}-v_{s}^{(p)}\right) / 2-2}(1+w) H_{v_{s}^{(p+1)}}(w) h_{v_{s}^{(p)}}(w) d w, \\
& a_{10}^{(p)}(s ; z)=z^{\mu+2}(1-z)^{\left(-v_{s}^{(p+1)}-v_{s}^{(p)}\right) / 2-1} h_{v_{s}^{(p+1)}}(z) h_{v_{s}^{(p)}}(z) \\
& -\int_{0}^{z} w^{\mu+1}(1-w)^{\left(-v_{s}^{(p+1)}-v_{s}^{(p)}\right) / 2-2}(1+w) h_{v_{s}^{(p+1)}}(w) h_{v_{s}^{(p)}}(w) d w, \\
& a_{01}^{(p)}(s ; z)=-z^{-\mu}(1-z)^{\left(v_{s}^{(p+1)}+v_{s}^{(p)}\right) / 2-1} H_{v_{s}^{(p+1)}}(z) H_{v_{s}^{(p)}}(z) \\
& +\int_{1}^{z} w^{-(\mu+1)}(1-w)^{\left(v_{s}^{(p+1)}+v_{s}^{(p)}\right) / 2-2}(1+w) H_{v_{s}^{(p+1)}}(w) H_{v_{s}^{(p)}}(w) d w, \\
& a_{11}^{(p)}(s ; z)=z(1-z)^{\left(-v_{s}^{(p+1)}+v_{s}^{(p)}\right) / 2-1} h_{v_{s}^{(p+1)}}(z) H_{v_{s}^{(p)}}(z) \\
& -\int_{0}^{z}(1-w)^{\left(-v_{s}^{(p+1)}+v_{s}^{(p)}\right) / 2-2}(1+w) h_{v_{s}^{(p+1)}}(w) H_{v_{s}^{(p)}}(w) d w .
\end{aligned}
$$

These integrals are convergent, and for $i=0,1$, we have

$$
\Phi^{(p)}(s ; z) \frac{d}{d z} \mathrm{~A}_{i}^{(p)}(s ; z)=c\left(v_{s}^{(p+1)}\right) \mathrm{U}_{i}^{(p)}(s ; z), \quad 0<z<1, s \in \tilde{D} .
$$

Proof. Lemma 3.7 shows that for a given compact set $U$ in $D$ there exists $\varepsilon>0$ such that $h_{s}(z)$ and $H_{s}(z)$ are bounded on $(0, \varepsilon] \cup[1-\varepsilon, 1)$ uniformly in $s \in U$. Hence the integrands of the formulas above except $a_{01}^{(p)}(s ; z)$ are bounded on $(0, \varepsilon]$ uniformly in $s \in U$, which implies the convergence of the integrals except $a_{01}^{(p)}(s ; z)$. To obtain the convergence of $a_{01}^{(p)}(s ; z)$ we need to assume $s \in \tilde{D}$.

We prove (35). Since $\Delta(z)=\operatorname{det} \Phi^{(p)}(s ; z)$ is the Wronskian for the fundamental solutions $\mathrm{F}_{i}^{(p)}(s ; z)$ of the differential equation (29) it satisfies the relation $(d / d z) \Delta(z)=\operatorname{tr}\left(\mathrm{B}^{(p)}(z)\right) \Delta(z)$. Since

$$
-\operatorname{tr}\left(\mathrm{B}^{(p)}(z)\right)=(n+1)(1-z)^{-1}+(\mu+1) z^{-1},
$$

we easily obtain $\Delta(z)=C_{s} z^{-(\mu+1)}(1-z)^{n+1}$ with some constant $C_{s}$. Using (33), we have

$$
\Delta(z)=-\frac{c\left(v_{s}^{p+1}\right)}{l p} z^{-(\mu+1)}(1+O(s ; z, z \log z))
$$


which implies that $C_{s}=-c\left(v_{s}^{(p+1)}\right) /(l p)$. Hence

$$
\Phi^{(p)}(s ; z)^{-1}=-\frac{l p}{c\left(v_{s}^{(p+1)}\right)} z^{\mu+1}(1-z)^{-(n+1)}\left[\begin{array}{cc}
f_{11}^{(p)} & -f_{01}^{(p)} \\
-f_{10}^{(p)} & f_{00}^{(p)}
\end{array}\right](s ; z) .
$$

Direct computation then gives $\frac{d}{d z} A_{i}^{(p)}(s ; z)=c\left(v_{s}^{(p+1)}\right) \Phi^{(p)}(s ; z)^{-1} U_{i}^{(p)}(s ; z)$.

Proposition 3.11. For $0<z<1$ and $s \in \tilde{D}$, set

$$
\tilde{\mathrm{F}}_{i}^{(p)}(s ; z)=\Phi^{(p)}(s ; z) \mathrm{A}_{i}^{(p)}(s ; z)=\mathrm{F}_{0}^{(p)}(s ; z) a_{0 i}^{(p)}(s ; z)+\mathrm{F}_{1}^{(p)}(s ; z) a_{1 i}^{(p)}(s ; z) .
$$

For each $i=0$, 1, the function $\tilde{\mathrm{F}}_{i}^{(p)}(s ; z)$ is a $C^{\infty}$-solution of the differential equation (23) satisfying the equalities

$$
\begin{aligned}
& \tilde{\mathrm{F}}_{0}^{(p)}(s ; z)=\frac{c\left(v_{s}^{(p+1)}\right)}{\mu+2}\left[\begin{array}{c}
1 /(l+m) \\
1
\end{array}\right] z\left(1+O\left(s ; z, z^{\mu+1} \log z\right)\right) \\
& \tilde{\mathrm{F}}_{1}^{(p)}(s ; z)=-\frac{c\left(v_{s}^{(p+1)}\right) c\left(v_{s}^{(p)}\right)}{\mu(\mu+1)}\left[\begin{array}{c}
- \\
\times(l+p+m) /(\operatorname{lp}(l+m)) \\
1
\end{array}\right] z^{-\mu} \\
& \times\left(1+O\left(s ; z, z^{\mu+1} \log z, z^{2 \mu+2}(\log z)^{2}\right)\right) .
\end{aligned}
$$

When $\operatorname{Re} s>0$, setting $y=1-z$, we have

$$
\begin{aligned}
& \tilde{\mathrm{F}}_{0}^{(p)}(s ; z)=\frac{c\left(v_{s}^{(p+1)}\right) c\left(v_{s}^{(p)}\right)}{v_{s}} \frac{-2}{v_{s}^{(p)}+l+m+1}\left[\begin{array}{c}
a_{0}(s) \\
1
\end{array}\right] y^{\left(-v_{s}^{(p)}+n\right) / 2}(1+O(s ; y)), \\
& \tilde{\mathrm{F}}_{1}^{(p)}(s ; z)=\frac{-2 c\left(v_{s}^{(p+1)}\right)}{-v_{s}^{(p)}+l+m+1}\left[\begin{array}{c}
a_{1}(s) \\
1
\end{array}\right] y^{\left(v_{s}^{(p)}+n\right) / 2}(1+O(s ; y))
\end{aligned}
$$

for holomorphic functions $a_{0}(s), a_{1}(s)$ on $\mathbb{C}$.

Proof. Lemma 3.4 combined with (35) shows that $\tilde{\mathrm{F}}_{i}^{(p)}(s ; z)$ is a solution of (23). The formulas (36) and (37) follow from (33), (34) combined with the asymptotics of $a_{i j}^{(p)}(s ; z)$ which is deduced from their explicit formula and Lemma 3.7.

Proposition 3.12. The functions $\mathrm{F}_{0}^{(p)}(s ; z), \mathrm{F}_{1}^{(p)}(s ; z), \tilde{\mathrm{F}}_{0}^{(p)}(s ; z)$ and $\tilde{\mathrm{F}}_{1}^{(p)}(s ; z)$ constructed above form a fundamental system of solutions of the differential equation (23) on $0<z<1$, which depend holomorphically on $s \in \tilde{D}$.

Proof. The solutions of (23) form a 4 dimensional $\mathbb{C}$-vector space. We already know that the functions $\mathrm{F}_{i}^{(p)}(s ; z)(i=0,1)$ and $\tilde{\mathrm{F}}_{i}^{(p)}(s ; z)(i=0,1)$ are solutions of (23) with linearly independent asymptotic behavior as $z \rightarrow+0$. 
The case $p=0$.

Proposition 3.13. For $i=0,1$, define $\tilde{\mathrm{F}}_{i}^{(0)}(s ; z), 0<z<1, s \in \tilde{D}$ by

$$
\begin{aligned}
& \tilde{\mathrm{F}}_{0}^{(0)}(s ; z)=(1-z)^{\left(-v_{s}^{(1)}+n\right) / 2}{ }_{2} F_{1}\left(-\frac{v_{s}^{(1)}-n}{2},-\frac{v_{s}^{(1)}+\lambda}{2}+1 ; \mu+1 ; z\right), \\
& \tilde{\mathrm{F}}_{1}^{(0)}(s ; z)=z^{-\mu}(1-z)^{\left(\nu_{s}^{(1)}+n\right) / 2}{ }_{2} F_{1}\left(\frac{v_{s}^{(1)}-n}{2}+1, \frac{v_{s}^{(1)}+\lambda}{2} ; v_{s}^{(1)}+1 ; 1-z\right) .
\end{aligned}
$$

The $\tilde{\mathrm{F}}_{i}^{(0)}(s ; z)$ form a fundamental system of $C^{\infty}$-solutions of $(32)$ such that

$$
\begin{aligned}
& \tilde{\mathrm{F}}_{0}^{(0)}(s ; z)=1+O(s ; z), \\
& \tilde{\mathrm{F}}_{1}^{(0)}(s ; z)=\frac{c\left(v_{s}^{(1)}\right)}{\mu(\mu+1)} \frac{v_{s}^{(1)}+n}{2} z^{-\mu}\left(1+O\left(s ; z, z^{\mu} \log z\right)\right),
\end{aligned}
$$

and such that for $\operatorname{Re} s>0$ and $y=1-z$

$$
\begin{aligned}
& \tilde{\mathrm{F}}_{0}^{(0)}(s ; z)=\frac{c\left(v_{s}^{(1)}\right)}{(\mu+1) v_{s}^{(1)}} \frac{v_{s}^{(1)}+n}{2} y^{\left(-v_{s}^{(1)}+n\right) / 2}(1+O(s ; y)), \\
& \tilde{\mathrm{F}}_{1}^{(0)}(s ; z)=y^{\left({ }_{s}^{(1)}+n\right) / 2}(1+O(s ; y)) .
\end{aligned}
$$

Proof of Theorem 3.2. First we construct the family of functions $\phi_{s}(s \in \tilde{D})$. By the general theory, the map $\psi:(h, t, k) \mapsto h a_{t} k$ from $H \times(0, \infty) \times K$ onto $G-H K$ is a submersion whose fibres are given as $\psi^{-1}\left(h a_{t} k\right)=\left\{\left(h m, t, m^{-1} k\right) \mid m \in M\right\}$. Hence we have a well-defined $C^{\infty}$-function $\phi_{s}^{(p)}: G-H K \rightarrow F_{p, p}$ for each $0<p \leqslant \mu$ by setting $\phi_{s}^{(p)}\left(h a_{t} k\right)=\frac{\mu(\mu+1) l p}{c\left(v_{s}^{(p+1)}\right) c\left(v_{s}^{(p)}\right)} \tau(k)^{-1}\left(\tilde{f}_{01}^{(p)}\left(s ; \tanh ^{2} t\right) \mathrm{v}_{0}^{(p)}+\tilde{f}_{11}^{(p)}\left(s ; \tanh ^{2} t\right) \mathrm{v}_{1}^{(p)}\right)$.

Here $\tilde{\mathrm{F}}_{1}^{(p)}(s ; z)$ is the column-matrix with entries $\tilde{f}_{01}^{(p)}(s ; z)$ and $\tilde{f}_{11}^{(p)}(s ; z)$, the solution of (23) constructed in Proposition 3.11. For $p=0$ we also have a welldefined $C^{\infty}$-function $\phi_{s}^{(0)}: G-H K \rightarrow \mathbb{C}$ such that

$$
\phi_{s}^{(0)}\left(h a_{t} k\right)=\frac{\mu(\mu+1)}{c\left(v_{s}^{(1)}\right)} \frac{2}{v_{s}^{(1)}+n} \tilde{\mathrm{F}}_{1}^{(0)}\left(s ; \tanh ^{2} t\right), \quad(h, t, k) \in H \times(0, \infty) \times K,
$$

where $\tilde{\mathrm{F}}_{1}^{(0)}(s ; z)$ is the function defined in Proposition 3.13. Define the function $\phi_{s}: G-H K \rightarrow \bigwedge^{\mu, \mu} \mathfrak{p}_{\mathbb{C}}^{*}$ by $\phi_{s}(g):=\sum_{p=0}^{\mu} \gamma_{p} L^{\mu-p}\left(\phi_{s}^{(p)}(g)\right)$ with $\left\{\gamma_{p}\right\}$ the family of real numbers in Proposition 1.4. Then, by Propositions 3.11 and 3.13, $\left\{\phi_{s}\right\}_{s \in \tilde{D}}$ has the required properties.

We prove the uniqueness of the family $\phi_{s}$. Assume we are given another family of functions $\varphi_{s}(s \in \tilde{D})$ possessing the same properties as $\phi_{s}$. Then we can write $\varphi_{s}(g)=\sum_{p=0}^{\mu} \gamma_{p} L^{\mu-p}\left(\varphi_{s}^{(p)}(g)\right)$ with $C^{\infty}$-functions $\varphi_{s}^{(p)}: G-H K \rightarrow F_{p, p}$ 
uniquely along the decomposition (27). We shall show that for each $0 \leqslant p \leqslant \mu$, $\phi_{s}^{(p)}(g)=\varphi_{s}^{(p)}(g)$ for all $g \in G-H K$ and $s \in \tilde{D}$; by condition (i) of Theorem 3.2, we have only to show it assuming $\operatorname{Re} s>n$.

First consider the case $p>0$. Then $\varphi_{s}^{(p)}\left(a_{t}\right)=g_{0}\left(\tanh ^{2} t\right) \mathrm{v}_{0}^{(p)}+g_{1}\left(\tanh ^{2} t\right) \mathrm{v}_{1}^{(p)}$ with a $C^{\infty}$-function $G(z)=\left[\begin{array}{l}g_{0}(z) \\ g_{1}(z)\end{array}\right]$ on $0<z<1$. Since $\varphi_{s}^{(p)}$ satisfies (22), the function $\mathrm{G}(z)$ is a solution of (23). Hence by Proposition 3.12, there are complex numbers $c_{0}, c_{1}, d_{0}, d_{1}$ such that

$$
\mathrm{G}(z)=c_{0} \mathrm{~F}_{0}^{(p)}(s ; z)+c_{1} \mathrm{~F}_{1}^{(p)}(s ; z)+d_{0} \tilde{\mathrm{F}}_{0}^{(p)}(s ; z)+d_{1} \tilde{\mathrm{F}}_{1}^{(p)}(s ; z) .
$$

We examine the behavior as $z \rightarrow+0$ on both sides of this identity to show $c_{1}=0$. We have $\lim _{z \rightarrow+0} z^{\mu+1} \mathrm{G}(z)=0$ by condition (iv). By Propositions 3.8 and 3.11 we have

$$
\begin{aligned}
& \lim _{z \rightarrow+0} z^{\mu+1} \mathrm{~F}_{0}^{(p)}(s ; z)=\lim _{z \rightarrow+0} z^{\mu+1} \tilde{\mathrm{F}}_{0}^{(p)}(s ; z)=\lim _{z \rightarrow+0} z^{\mu+1} \tilde{\mathrm{F}}_{1}^{(p)}(s ; z)=0, \\
& \lim _{z \rightarrow+0} z^{\mu+1} \mathrm{~F}_{1}^{(p)}(s ; z) \neq 0 .
\end{aligned}
$$

Hence $c_{1}=0$ and $c_{0} \mathrm{~F}_{0}^{(p)}+d_{0} \tilde{\mathrm{F}}_{0}^{(p)}+d_{1} \tilde{\mathrm{F}}_{1}^{(p)}=\mathrm{G}$. Similarly we can use the behavior as $y=1-z \rightarrow+0$ to conclude $c_{0}=d_{0}=0$. Indeed, since we assume $\operatorname{Re} s>n$, we have $\lim _{t \rightarrow+\infty} e^{\left(-v_{s}^{(p)}+n\right) t} \mathrm{G}\left(\tanh ^{2} t\right)=0$ by condition $(\mathrm{v})$, and

$$
\lim _{t \rightarrow+\infty} e^{\left(-v_{s}^{(p)}+n\right) t} \mathrm{~F}_{0}^{(p)}\left(s ; \tanh ^{2} t\right)=\lim _{t \rightarrow+\infty} e^{\left(-v_{s}^{(p)}+n\right) t} \tilde{\mathrm{F}}_{1}^{(p)}\left(s ; \tanh ^{2} t\right)=0
$$

by Propositions 3.8 and 3.11. Hence $d_{0}=0$ and $c_{0} \mathrm{~F}_{0}^{(p)}+d_{1} \tilde{\mathrm{F}}_{1}^{(p)}=\mathrm{G}$. Since $\operatorname{Re} v_{s}^{(p)}>\operatorname{Re} v_{s}^{(p+1)}>\operatorname{Re} s>n$ (see Lemma 3.1), we have

$$
\lim _{t \rightarrow+\infty} e^{\left(-\nu_{s}^{(p+1)}+n\right) t} \tilde{\mathrm{F}}_{1}^{(p)}\left(s ; \tanh ^{2} t\right)=\lim _{t \rightarrow+\infty} e^{\left(-\nu_{s}^{(p+1)}+n\right) t} \mathrm{G}\left(\tanh ^{2} t\right)=0
$$

by condition (v) and Proposition 3.11. Hence $c_{0}=0$.

Consequently, $\mathrm{G}=d_{1} \tilde{\mathrm{F}}_{1}^{(p)}$. By condition (iv) and Proposition 1.3, we have

$$
\lim _{z \rightarrow+0} z^{\mu} \mathrm{G}(z)=\left[\begin{array}{c}
m(l+p+m) /(l+m) \\
-l p
\end{array}\right] .
$$

At the same time, Proposition 3.11 gives

$$
\lim _{z \rightarrow+0} z^{\mu} \tilde{\mathrm{F}}_{1}^{(p)}(s ; z)=\frac{c\left(v_{s}^{(p+1)}\right) c\left(v_{s}^{(p)}\right)}{\mu(\mu+1) l p}\left[\begin{array}{c}
m(l+p+m) /(l+m) \\
-l p
\end{array}\right],
$$

so the constant $d_{1}$ equals $\mu(\mu+1) l p /\left(c\left(v_{s}^{(p+1)}\right) c\left(v_{s}^{(p)}\right)\right)$.

From the definition of $\phi_{s}^{(p)}$, we have $\varphi_{s}^{(p)}\left(a_{t}\right)=\phi_{s}^{(p)}\left(a_{t}\right)$. This is sufficient to conclude $\varphi_{s}^{(p)}(g)=\phi_{s}^{(p)}(g)$ for all $g \in G-H K$ by the $(H, K)$-equivariance 
condition. The discussion for the case $p=0$ is quite similar. This completes the proof of uniqueness.

\section{Some properties of the secondary spherical function.}

Theorem 3.14. Let $\phi_{s}(s \in \tilde{D})$ be the secondary spherical function constructed in Theorem 3.2.

(a) There exist $\mu$ polynomial functions $\mathrm{a}_{\alpha}(s)(0 \leqslant \alpha \leqslant \mu-1)$ with values in $\left(\bigwedge^{\mu, \mu} \mathfrak{p}_{\mathbb{C}}^{*}\right)^{M}$, positive number $\varepsilon$ and $\left(\bigwedge^{\mu, \mu} \mathfrak{p}_{\mathbb{C}}^{*}\right)^{M}$-valued holomorphic functions $\mathrm{b}_{i(s, z)}(i=0,1,2)$ on $\{(s, z)|s \in \tilde{D}| z \mid,<\varepsilon\}$ such that

$$
\mathrm{a}_{0}(s)=\mathrm{w}, \quad \mathrm{a}_{\alpha}(-s)=\mathrm{a}_{\alpha}(s), \quad \operatorname{deg}\left(\mathrm{a}_{\alpha}(s)\right) \leqslant 2 \alpha,
$$

and such that for $s \in \tilde{D}, z=\tanh ^{2} t \in(0, \varepsilon)$,

$$
\phi_{s}\left(a_{t}\right)=\sum_{\alpha=0}^{\mu-1} \frac{\mathrm{a}_{\alpha}(s)}{z^{\mu-\alpha}}+\mathrm{b}_{0}(s ; z)+\mathrm{b}_{1}(s ; z) \log z+\mathrm{b}_{2}(s ; z) z^{\mu+2}(\log z)^{2} .
$$

(b) There exists a positive number $\varepsilon^{\prime}$ and $\left(\bigwedge^{\mu, \mu} \mathfrak{p}_{\mathbb{C}}^{*}\right)^{M}$-valued holomorphic functions $\mathrm{f}^{(p)}(s ; y)(0 \leqslant p \leqslant \mu)$ on $\left\{(s, y)|| y \mid<\varepsilon^{\prime}, \operatorname{Re} s>n\right\}$ such that

$$
\phi_{s}\left(a_{t}\right)=\sum_{p=0}^{\mu} y^{\left(v_{s}^{(p)}+n\right) / 2} \mathrm{f}^{(p)}(s ; y), \quad \operatorname{Re} s>n, y=\frac{1}{\cosh ^{2} t} \in\left(0, \varepsilon^{\prime}\right) .
$$

(c) For any differential operator with holomorphic coefficient $\partial_{s}$ on $\tilde{D}$, the function $\partial_{s} \phi_{s}(g)$ on $G-H K$ belongs to $C_{\tau}^{\infty}$.

Proof. (a) Recall the construction of $\phi_{s}$. Then (36) and (38) immediately yields the existence of the expression of the form (40) except the nature of the functions $\mathrm{a}_{\alpha}(s)$. We have $\mathrm{a}_{0}(s)=\mathrm{w}$ from condition (iv). Hence it remains to show that $\mathrm{a}_{\alpha}(s)$ is an even polynomial function with degree no more than $2 \alpha$. For that purpose, we examine the differential equation (22) again. By Theorem 2.3, equation (22) can be written as

$$
\left(\frac{d^{2}}{d z^{2}}+\left(\frac{\mu+1}{z}+\frac{n-1}{1-z}\right) \frac{d}{d z}+2(s ; z)\right) \phi_{s}=0
$$

with

$$
2(s ; z)=\frac{-\mathscr{S}}{z(1-z)}+\frac{\mathscr{S}^{\prime}}{z^{2}(1-z)}+\frac{\tau\left(\Omega_{M_{0}}\right)+\lambda^{2}-s^{2}}{4 z(1-z)^{2}} .
$$

We have

$$
2(s ; z)=\frac{\mathscr{S}^{\prime}}{z^{2}}+\sum_{\gamma=-1}^{\infty} 2_{\gamma}(s) z^{\gamma}, \quad|z|<1,
$$


with

$$
2_{\gamma}(s)=-\mathscr{Y}+\mathscr{Y}^{\prime}+\frac{1}{4}(\gamma+2)\left(\lambda^{2}-s^{2}+\tau\left(\Omega_{M_{0}}\right)\right), \quad \gamma \geqslant-1 .
$$

Substitute this expression of $2(s ; z)$ and (40) to the left-hand side of (42) and compute the coefficient of $z^{-\mu+\alpha-2}$. Since it should be zero, we obtain the recurrence relation among the tensors $\mathrm{a}_{\alpha}(s)$ : for $0<\alpha \leqslant \mu-1$,

$$
\left(\mathscr{Y}^{\prime}-\alpha(\mu-\alpha)\right) \mathrm{a}_{\alpha}(s)=-\sum_{\gamma=0}^{\alpha-1}\left(2_{\alpha-\gamma-2}(s)-(n-1)(\mu-\gamma)\right) \mathrm{a}_{\gamma}(s) .
$$

By (17), the operator $\mathscr{S}^{\prime}$ preserves the subspaces $L^{\mu-p}\left(F_{p, p}^{M}\right)(0<p \leqslant \mu)$ and the restriction of $\mathscr{Y}^{\prime}$ is represented with respect to the basis $L^{\mu-p}\left(\mathrm{v}_{i}^{(p)}\right)(i=0,1)$ by

$$
\mathrm{Q}_{-2}^{(p)}=\left[\begin{array}{cc}
-\frac{p l}{l+m} & -\frac{m(l+p+m)}{(l+m)^{2}} \\
-l p & -\frac{m(l+p+m)}{l+m}
\end{array}\right] .
$$

Since $\operatorname{det}\left(\mathrm{Q}_{-2}^{(p)}-\alpha(\mu-\alpha)\right)=\alpha(\alpha+1)(\alpha-\mu-1)(\alpha-\mu)$ is not zero for $0<\alpha \leqslant \mu-1$, the operator $\mathscr{S}^{\prime}-\alpha(\mu-\alpha)$ is invertible on $\left(\wedge^{\mu, \mu} \mathfrak{p}_{\mathbb{C}}^{*}\right)^{M}$. Therefore we can determine $\mathrm{a}_{\alpha}(s)$ uniquely by the recurrence relation (43), from which we easily know that $\mathrm{a}_{\alpha}(s)$ is an even polynomial function with degree no more than $2 \alpha$.

By the construction of $\phi_{s}$, part (c) is obvious. Part (b) follows from (37) and (39).

The function $\psi_{s}$. For $s \in \tilde{D}$, define the function $\psi_{s}: G-H K \rightarrow \bigwedge^{r, r} \mathfrak{p}_{\mathbb{C}}^{*}$ by

$$
\psi_{s}(g)=\nabla_{-} \nabla_{+} \phi_{s}(g), \quad g \in G-H K .
$$

We easily see that $\psi_{s}$ has the same properties as (2) and (3) of the previous theorem for $\phi_{s}$. It also behaves similarly to (40) for $\phi_{s}$ near $t=0$,

$$
\psi_{s}\left(a_{t}\right)=\sum_{\alpha=0}^{\mu-1} \frac{c_{\alpha}(s)}{z^{\mu-\alpha}}+O\left(s ; 1, \log z, z^{\mu}(\log z)^{2}\right),
$$

except that the degree of the $\left(\bigwedge^{r, r} \mathfrak{p}_{\mathbb{C}}^{*}\right)^{M}$-valued polynomial $c_{\alpha}(s)$ is no more than $2(\alpha+1)$. Indeed by applying the expression (24) of the Schmid operator to (40) we can obtain the required expression with one extra term of the form $u z^{-(\mu+1)}$. The only thing we have to do here is to show $u=0$. By a direct computation, we have $\mathrm{u}=\left(\mu(\mu+1) e\left(\omega_{0} \wedge \bar{\omega}_{0}\right)+\mu \mathscr{A}+\mathscr{R}_{-} \mathscr{R}_{+}\right) \mathrm{w}$. Since $\mathrm{w}$ is $(H \cap K)$-invariant and the elements $\bar{Z}_{j}, Z_{j}$ with $n-r+1 \leqslant j \leqslant n-1$ belong to $(\mathfrak{k} \cap \mathfrak{h})_{\mathbb{C}}$, we have $\mathscr{R}_{ \pm} \mathrm{w}=0$. Hence $\mathrm{u}=\mu(2 / \sqrt{-1})\left(\eta-(\sqrt{-1} / 2) \mu \omega_{0} \wedge \bar{\omega}_{0}\right) \wedge \mathrm{w}$. By Proposition 1.4 , the right-hand side of this identity is zero. 


\section{Poincaré series}

Let $\Gamma$ be a discrete subgroup of $G$. We assume that $(G, H, \Gamma)$ is arranged as follows. There exists a connected reductive $\mathbb{Q}$-group $G$, a closed $\mathbb{Q}$-subgroup $\mathrm{H}$ of $\mathrm{G}$ and an arithmetic subgroup $\Delta$ of $\mathrm{G}(\mathbb{Q})$ such that there exists a morphism of Lie groups from $\mathrm{G}(\mathbb{R})$ onto $G$ with compact kernel which maps $\mathrm{H}(\mathbb{R})$ onto $H$ and $\Delta$ onto $\Gamma$. Set $K_{H}=H \cap K, \Gamma_{H}=\Gamma \cap H$.

Invariant measures. Let $d k$ and $d k_{0}$ be the Haar measures of compact groups $K$ and $K_{H}$ with total volume 1 . There is a unique Haar measure $d g$ on $G$ such that the quotient measure $d g / d k$ corresponds to the measure on the symmetric space $G / K$ determined by the invariant volume form vol. We define $d h$ on $H$ analogously: $d h / d k_{0}$ corresponds to the measure on $H / K_{H}$ determined by vol $H$.

Lemma 4.1. For any measurable functions $f$ on $G$ we have

$$
\int_{G} f(g) d g=\int_{H} d h \int_{K} d k \int_{0}^{\infty} f\left(h a_{t} k\right) \varrho(t) d t
$$

with $d t$ the usual Lebesgue measure on $\mathbb{R}$ and

$$
\varrho(t)=2 c_{r}(\sinh t)^{2 r-1}(\cosh t)^{2 n-2 r+1}, \quad c_{r}=\pi^{r} / \mu ! .
$$

Proof. For closed subgroups $Q_{1} \subset Q_{2}$ of $G$ with Lie algebras $\mathfrak{q}_{i}=\operatorname{Lie}\left(Q_{i}\right)$ for $i=1,2$, we regard $\left(\mathfrak{q}_{2} / \mathfrak{q}_{1}\right)^{*} \subset \mathfrak{g}^{*}$ by the dual map of the composite of the orthogonal projection $\mathfrak{g} \rightarrow \mathfrak{q}_{2}$ and the canonical surjection $\mathfrak{q}_{2} \rightarrow \mathfrak{q}_{2} / \mathfrak{q}_{1}$. Let $\operatorname{vol}_{\mathfrak{q}_{2} / \mathfrak{q}_{1}}$ be the element $\xi_{1} \wedge \cdots \wedge \xi_{s} \in \wedge\left(\mathfrak{q}_{2} / \mathfrak{q}_{1}\right)^{*}$ with $\left\{\xi_{i}\right\}$ any orthonormal basis of $\left(\mathfrak{q}_{2} / \mathfrak{q}_{1}\right)^{*}$. Assume $Q_{1}$ is compact. Then there exists a unique left $Q_{2}$-invariant $s$-form $\mathrm{Z}_{Q_{2} / Q_{1}}$ on $Q_{2} / Q_{1}$ whose value at $o=e Q_{2}$ is $\operatorname{vol}_{\mathfrak{q}_{2} / \mathfrak{q}_{1}}$. Let $d Z_{Q_{2} / Q_{1}}$ be the $Q_{2}$-invariant measure on $Q_{2} / Q_{1}$ corresponding to $\mathrm{Z}_{Q_{2} / Q_{1}}$. For example, vol $\mathfrak{g}_{\mathfrak{g} / \mathfrak{k}}=$ vol and $\operatorname{vol}_{\mathfrak{h} / \mathfrak{m}}=\operatorname{vol}_{H} \wedge \operatorname{vol}_{\mathfrak{h} \cap \mathfrak{k} / \mathfrak{m} .}$

The decomposition $G=H A K$ yields the diffeomorphism $j$ from $H / M \times(0, \infty)$ to $(G-H K) / K$ defined by $j(\dot{h}, t)=h a_{t} K(\dot{h} \in H / M, t>0)$; see [Heckman and Schlichtkrull 1994, Theorem 2.4, p. 108]. A simple computation with the aid of the formulas in the proof of Lemma 2.2 proves the identity

$$
j^{*} \mathrm{Z}_{G / K}=2(\sinh t)^{2 r-1}(\cosh t)^{2 n-2 r+1} \mathrm{Z}_{H / M} \wedge d t .
$$

Hence under the identification $H / M \times(0, \infty)=(G-H K) / K$ we have

$$
d Z_{G / K}(\dot{g})=2(\sinh t)^{2 r-1}(\cosh t)^{2 n-s r+1} d Z_{H / M}(\dot{h}) d t .
$$

The measure $d Z_{G / K}(\dot{g})$ is precisely $d g / d k$ in our normalization. Let $d m$ be the Haar measure of $M$ with total volume one. The resulting quotient measure $d h / d m$ 
on $H / M$ should be proportional to $d Z_{H / M}(\dot{h})$. We determine the proportionality constant $C_{0}$ in such a way that

$$
\frac{d h}{d m}=C_{0} d Z_{H / M}(\dot{h}) .
$$

We have a decomposition

$$
d Z_{H / M}(\dot{h})=\frac{d h}{d k_{0}} d Z_{H \cap K / M}\left(\dot{k}_{0}\right),
$$

since $d Z_{H / H \cap K}(\dot{h})=d h / d k_{0}$. Using the relation $d h / d m=\left(d h / d k_{0}\right)\left(d k_{0} / d m\right)$, it follows that

$$
\frac{d k_{0}}{d m}=C_{0} d Z_{H \cap K / M}\left(\dot{k}_{0}\right) .
$$

Since the total measure of $H \cap K / M$ with respect to $d k_{0} / d m$ is 1 , we then obtain the equality $C_{0}^{-1}=\int_{H \cap K / M} d Z_{H \cap K / M}\left(\dot{k}_{0}\right)$. To compute this integral, we use the diffeomorphism $\Pi: H \cap K / M \rightarrow \mathbb{S}^{2 r-1}$ defined by

$$
\Pi\left(\operatorname{diag}\left(u_{1}, u_{2}, u_{0}\right) M\right)=u_{0}^{-1} u_{2}(\mathrm{e}), \quad\left(u_{1}, u_{2}, u_{0}\right) \in \mathrm{U}(n-r) \times \mathrm{U}(r) \times \mathrm{U}(1),
$$

with $\mathbb{S}^{2 r-1}:=\left\{\left.z \in \mathbb{C}^{r}\left|\sum_{i=1}^{r}\right| z_{i}\right|^{2}=1\right\}$ and $\mathrm{e}:={ }^{t}(0, \ldots, 0,1) \in \mathbb{S}^{2 r-1}$. Thus, easily, the pullback of the volume form $Z_{\mathbb{S}^{2 r-1}}=\sum_{i=1}^{2 r}(-1)^{i-1} x_{i} d x_{1} \wedge \cdots \wedge \hat{d} x_{i} \wedge \ldots d x_{2 r}$ of the sphere $\mathbb{S}^{2 r-1}$ is $2 Z_{H \cap K / M}$. Hence

$$
C_{0}^{-1}=\int_{H \cap K / M} d Z_{H \cap K / M}\left(\dot{k}_{0}\right)=\frac{1}{2} \int_{\mathbb{S}^{2 r-1}} d Z_{\mathbb{S}^{2 r-1}}=\frac{\pi^{r}}{\Gamma(r)} .
$$

Using (45), (46) and the value of $C_{0}$ just obtained, the required integration formula easily follows.

Currents defined by Poincaré series. Let $\mathfrak{F}$ denote the set of the families of functions $\left\{\varphi_{s}\right\}_{s \in \tilde{D}}$ such that $\varphi_{s}=\partial_{s} \phi_{s}(s \in \tilde{D})$ or $\varphi_{s}=\partial_{s} \psi_{s}(s \in \tilde{D})$ with some differential operator $\partial_{s}$ with holomorphic coefficient on $\tilde{D}$.

For $\left\{\varphi_{s}\right\} \in \mathfrak{F}$, introduce the Poincaré series

$$
\tilde{P}\left(\varphi_{s}\right)(g)=\sum_{\gamma \in \Gamma_{H} \backslash \Gamma} \varphi_{s}(\gamma g), \quad g \in G,
$$

which is the most basic object in our investigation. First of all, we discuss its convergence in a weak sense. Note that $\varphi_{s}$ takes its values in a finite dimensional Hilbert space $\wedge \mathfrak{p}_{\mathbb{C}}^{*}$ with the norm $\|\alpha\|=(\alpha \mid \alpha)^{1 / 2}$.

Theorem 4.2. The function in s defined by the integral

$$
\tilde{P}\left(\left\|\varphi_{s}\right\|\right):=\int_{\Gamma \backslash G}\left(\sum_{\gamma \in \Gamma_{H} \backslash \Gamma}\left\|\varphi_{s}(\gamma g)\right\|\right) d \dot{g}
$$


is locally bounded on $\operatorname{Re} s>n$. For each $s$ with $\operatorname{Re} s>n$, the series (47) converges absolutely almost everywhere in $g \in G$ to define an $L^{1}$-function on $\Gamma \backslash G$.

Proof. We assume $\varphi_{s}=\partial \phi_{s}$. (The proof for $\partial \psi_{s}$ is the same.) Let $U$ be a compact subset of $\operatorname{Re} s>n$. Then by Theorem 3.14 and Lemma 3.1, there exist positive numbers $a, \delta$ and $C_{0}$ such that

$$
\begin{array}{ll}
\left\|\varphi_{s}\left(a_{t}\right)\right\| \leqslant C_{0} t^{-2 \mu}, & (s, t) \in U \times(0, a], \\
\left\|\varphi_{s}\left(a_{t}\right)\right\| \leqslant C_{0} e^{-t(2 n+\delta)}, & (s, t) \in U \times[a, \infty) .
\end{array}
$$

From the form of $\varrho(t)$, we can find a positive constant $C_{1}$ such that

$$
\varrho(t) \leqslant C_{1} t^{2 \mu+1} \text { for } t \in(0, a] \text { and } \varrho(t) \leqslant C_{1} e^{2 n t} \text { for } t \in[a, \infty) .
$$

First as in the proof of [Oda and Tsuzuki 2003, Proposition 3.1.1] and then by using the estimations above, we obtain the uniform bound of $\tilde{P}\left(\left\|\varphi_{s}\right\|\right)$ for $s \in U$.

If $\Gamma$ is neat, then the quotient space $\Gamma \backslash G / K$ acquires a structure of complex manifold from the one on $G / K \cong \mathfrak{D}$. Let $\pi: G / K \rightarrow \Gamma \backslash G / K$ be the natural projection. Let $A(\Gamma \backslash G / K)$ denote the space of $C^{\infty}$-differential forms on $\Gamma \backslash G / K$ and $A_{\mathrm{c}}(\Gamma \backslash G / K)$ the subspace of compactly supported forms. Given $\alpha \in A(\Gamma \backslash G / K)$, there is a unique $C^{\infty}$-function $\tilde{\alpha}: G \rightarrow \wedge \mathfrak{p}_{\mathbb{C}}^{*}$ such that $\tilde{\alpha}(\gamma g k)=\tau(k)^{-1} \tilde{\alpha}(g)$ for $\gamma \in \Gamma$ and $k \in K$ and such that

$$
\left\langle\left(\pi^{*} \alpha\right)(g K), d L_{g}\left(\xi_{o}\right)\right\rangle=\left\langle\tilde{\alpha}(g), \xi_{o}\right\rangle, \quad g \in G, \xi_{o} \in \bigwedge \mathfrak{p}=\bigwedge \mathrm{T}_{o}(G / K) .
$$

Here $L_{g}$ denotes the left translation on $G / K$ by the element $g$ and we identify $\mathfrak{p}$ with $\mathrm{T}_{o}(G / K)$, the tangent space of $G / K$ at $o=e K$. Then for any $\alpha, \beta \in$ $A(\Gamma \backslash G / K)$, we have

$$
\int_{\Gamma \backslash G / K} \alpha \wedge * \bar{\beta}=\int_{\Gamma \backslash G}(\tilde{\alpha}(g) \mid \tilde{\beta}(g)) d \dot{g} .
$$

For any left $\Gamma$-invariant function $f$ on $G$, the integral

$$
\mathscr{F}_{H}(f ; g)=\int_{\Gamma_{H} \backslash H} f(h g) d h, \quad g \in G,
$$

plays a fundamental role in our further study. We already discussed the convergence problem of this integral in [Oda and Tsuzuki 2003, §3.2]. For convenience we recall the result. If $\Gamma$ is cocompact, we take a compact fundamental domain $\mathfrak{S}^{1}$ for $\Gamma$ in $G$ and $t_{\mathfrak{S}^{1}}$ the constant function 1 . Hence $G=\Gamma \mathfrak{S}^{1}$ in this case. If $\Gamma$ is not cocompact, then one can fix a complete set of representatives $\mathrm{P}^{i}(1 \leqslant i \leqslant h)$ of $\Delta$ conjugacy classes of $\mathbb{Q}$-parabolic subgroups in $G$ together with $\mathbb{Q}$-split tori $\mathbb{G}_{m} \cong \mathrm{A}^{i}$ in the radical of $\mathrm{P}^{i}$ such that an eigencharacter of $\operatorname{Ad}(t)\left(t \in \mathbb{G}_{m}\right)$ in the Lie algebra of $\mathrm{P}^{i}$ is one of $t^{j}(j=0,1,2)$. For each $i$, let $T^{i}$ and $N^{i}$ be the images in $G$ of $\mathrm{A}^{i}(\mathbb{R})$ and the unipotent radical of $\mathrm{P}^{i}(\mathbb{R})$ respectively. Then we can choose a Siegel 
domain $\mathfrak{S}^{i}$ in $G$ with respect to the Iwasawa decomposition $G=N^{i} T^{i} K$ for each $i$ such that $G$ is a union of $\Gamma \mathfrak{S}^{i}(1 \leqslant i \leqslant h)$. Let $t_{\mathfrak{S}^{i}}: \mathfrak{S}^{i} \rightarrow(0, \infty)$ be the function $t_{\mathfrak{S}^{i}}\left(n_{i} \underline{t}_{i} k\right)=t\left(n_{i} \underline{t}_{i} k \in \mathfrak{S}^{i}\right)$. Here $\underline{t}_{i}$ denotes the image of $t \in \mathbb{G}_{m}(\mathbb{R}) \cong \mathrm{A}^{i}(\mathbb{R})$ in $T^{i}$.

Given $\delta \in\left(2 r n^{-1}, 1\right)$, let $\mathfrak{M}_{\delta}$ be the space of all left $\Gamma$-invariant $C^{\infty}$-functions $f: G \rightarrow \wedge \mathfrak{p}_{\mathbb{C}}^{*}$ with the $K$-equivariance $f(g k)=\tau(k)^{-1} f(g)$ such that, for any $\varepsilon \in(0, \delta)$ and $D \in U\left(\mathfrak{g}_{\mathbb{C}}\right)$, we have

$$
\left\|R_{D} \varphi(g)\right\| \prec t_{\mathfrak{S}^{i}}(g)^{(2-\varepsilon) n}, \quad g \in \mathfrak{S}^{i}, 1 \leqslant i \leqslant h .
$$

Proposition 4.3. Let $f \in \mathfrak{M}_{\delta}$ with $\delta \in\left(2 r n^{-1}, 1\right)$ and $D \in U\left(\mathfrak{g}_{\mathbb{C}}\right)$.

(1) For any $\varepsilon \in\left(2 r n^{-1}, \delta\right)$

$$
\mathscr{J}_{H}\left(\left\|R_{D} f\right\| ; a_{t}\right) \prec e^{(2-\varepsilon) n t}, \quad t \geqslant 0 .
$$

The function $\mathscr{F}_{H}(f ; g)$ is of class $C^{\infty}$, belongs to $C_{\tau}^{\infty}$ and

$$
\mathscr{F}_{H}\left(R_{D} f ; g\right)=R_{D} \mathscr{F}_{H}(f ; g), \quad g \in G .
$$

(2) For any $\left\{\varphi_{s}\right\} \in \mathfrak{F}$, the integral

$$
\int_{\Gamma \backslash G}\left|\left(\tilde{P}\left(\varphi_{s}\right)(g) \mid R_{D} f(g)\right)\right| d \dot{g}
$$

is finite if $\operatorname{Re} s>3 n-2 r$, and

$$
\int_{\Gamma \backslash G}\left(\tilde{P}\left(\varphi_{s}\right)(g) \mid R_{D} f(g)\right) d \dot{g}=\int_{0}^{\infty} \varrho(t)\left(\varphi_{s}\left(a_{t}\right) \mid R_{D} \mathscr{F}_{H}\left(f ; a_{t}\right)\right) d t .
$$

Proof. See [Oda and Tsuzuki 2003, Theorem 3.2.1] and its proof. The number $\tau_{\mathbb{Q}}(G, \sigma)$ is $2\left(1-r n^{-1}\right)$ in our setting here.

Proposition 4.4. There exists a unique current $P\left(\varphi_{s}\right)$ on $\Gamma \backslash G / K$ such that for $\alpha \in A_{\mathrm{c}}(\Gamma \backslash G / K)$,

$$
\left\langle P\left(\varphi_{s}\right), * \bar{\alpha}\right\rangle=\int_{\Gamma \backslash G}\left(\tilde{P}\left(\varphi_{s}\right)(g) \mid \tilde{\alpha}(g)\right) d \dot{g}=\int_{0}^{\infty} \varrho(t)\left(\varphi_{s}\left(a_{t}\right) \mid \mathscr{F}_{H}\left(\tilde{\alpha} ; a_{t}\right)\right) d t .
$$

Let $\partial_{s}$ be a holomorphic differential operator on $\tilde{D}$. Then for any $\alpha \in A_{\mathrm{c}}(\Gamma \backslash G / K)$, the function $s \mapsto\left\langle P\left(\varphi_{s}\right), \alpha\right\rangle$ is holomorphic on $\tilde{D}$ and $\partial_{s}\left\langle P\left(\varphi_{s}\right), \alpha\right\rangle=\left\langle P\left(\partial_{s} \varphi_{s}\right), \alpha\right\rangle$.

Proof. The $L^{1}$-function $\tilde{P}\left(\varphi_{s}\right)$ on $\Gamma \backslash G$ satisfies $\tilde{P}\left(\varphi_{s}\right)(g k)=\tau(k)^{-1} \tilde{P}\left(\varphi_{s}\right)(g)$ for all $k \in K$. Hence it defines an $L^{1}$-form $P\left(\varphi_{s}\right)$ on $\Gamma \backslash G / K$ naturally. Then the linear functional $\alpha \mapsto \int_{\Gamma \backslash G / K} P\left(\varphi_{s}\right) \wedge \alpha$ on $A_{\mathrm{c}}(\Gamma \backslash G / K)$ is a current, which we also denote by $P\left(\varphi_{s}\right)$. The first equality of (50) follows from (49). Let $\alpha \in$ $A_{\mathrm{c}}(\Gamma \backslash G / K)$. Since $\tilde{\alpha}$ is left $\Gamma$-invariant and has compact support mod $\Gamma$, the image $\tilde{\alpha}(\Gamma \backslash G)$ is a compact subset of $\wedge \mathfrak{p}_{\mathbb{C}}^{*}$. Hence there is a positive constant $C_{0}$ 
such that $|(\mathrm{u} \mid \tilde{\alpha}(g))| \leqslant C_{0}\|\mathrm{u}\|$ for all $\mathrm{u} \in \bigwedge \mathfrak{p}_{\mathbb{C}}^{*}$ and $g \in G$. Then for any loop $C$ in $\operatorname{Re} s>n$, we have

$$
\begin{aligned}
\int_{C} d s \int_{\Gamma \backslash G} \sum_{\gamma \in \Gamma_{H} \backslash \Gamma}\left|\left(\varphi_{s}(\gamma g) \mid \tilde{\alpha}(\gamma g)\right)\right| d \dot{g} & \leqslant C_{0} \int_{C} d s \int_{\Gamma \backslash G}\left(\sum_{\gamma \in \Gamma_{H} \backslash \Gamma}\left\|\varphi_{s}(\gamma g)\right\|\right) d \dot{g} \\
& =C_{0} \int_{C} \tilde{P}\left(\left\|\varphi_{s}\right\|\right) d s .
\end{aligned}
$$

By Theorem 4.2, the last integral in $s$ is finite. Hence by Fubini's theorem and by the holomorphicity of each function $s \mapsto\left(\varphi_{s}(g) \mid \tilde{\alpha}(g)\right)$, we have

$$
\int_{C} d s \int_{\Gamma \backslash G}\left(\tilde{P}\left(\varphi_{s}\right)(g) \mid \tilde{\alpha}(g)\right) d \dot{g}=0 .
$$

Since $C$ is arbitrary, the holomorphicity in $s$ of the integral (50) follows. The second equality of (50) follows by a standard computation.

Differential equation of functions related to $\tilde{\boldsymbol{G}}_{\boldsymbol{s}}$. For each $0 \leqslant j \leqslant \mu$, put $\boldsymbol{\delta}_{j, s} \tilde{\boldsymbol{G}}_{s}=$ $\left(c_{r} \mu \mu !\right)^{-1} \tilde{P}\left(\delta_{s, j} \phi_{s}\right)$ with

$$
\boldsymbol{\delta}_{j, s}=\frac{1}{j !}\left(\frac{1}{2 s} \frac{d}{d s}\right)^{j}, \quad j \in \mathbb{N},
$$

which we will regard as a $\bigwedge^{\mu, \mu} \mathfrak{p}_{\mathbb{C}}^{*}$-valued $L^{1}$-function on $\Gamma \backslash G$.

Theorem 4.5. Let $0 \leqslant j \leqslant \mu$ be an integer. Suppose $\operatorname{Re} s>3 n-2 r$. Then for any $f \in \mathfrak{M}_{\delta}$ with $\delta \in\left(2 r n^{-1}, 1\right)$, we have

$$
\int_{\Gamma \backslash G}\left(\delta_{j, s} \tilde{G}_{s}(g) \mid R_{\left(\Omega+\lambda^{2}-\bar{s}^{2}\right)^{j+1}} f(g)\right) d \dot{g}=\frac{4}{\mu !}\left(\mathrm{w} \mid \mathscr{E}_{H}(f ; e)\right) .
$$

Proof. For $f \in \mathfrak{M}_{\delta}$, let $I_{j}(f)$ denote the integral above. Then we shall prove $I_{j}(f)=I_{j-1}(f)$ for $1 \leqslant j \leqslant \mu$ and $I_{0}(f)=\frac{4}{\mu !}\left(\mathrm{w} \mid \mathscr{E}_{H}(f ; e)\right)$, which obviously imply the equality. Since we already have Proposition 4.3, we can set aside the convergence argument of various integrals in these formulas. Fix $0 \leqslant j \leqslant \mu$ and put

$$
\tilde{F}(g)=\mathscr{F}_{H}\left(R_{\left(\Omega-s^{2}+\lambda^{2}\right)^{j}} f ; g\right), \quad g \in G .
$$

Since $\tilde{F} \in C_{\tau}^{\infty}$, (14) implies

$$
R_{\left(\Omega+\lambda^{2}-\bar{s}^{2}\right)} \tilde{F}\left(a_{t}\right)=\left(\mathscr{D}+\lambda^{2}-\bar{s}^{2}\right) F(t)
$$

with $\mathscr{D}$ the differential operator in $t>0$ given by the formula inside the bracket of (14), and $F(t)=\tilde{F}\left(a_{t}\right)$. By Proposition 4.4(2), we have

$$
I_{j}(f)=\frac{1}{c_{r} \mu \mu !} \int_{0}^{\infty} \varrho(t)\left(\delta_{j, s} \phi_{s}\left(a_{t}\right) \mid\left(\mathscr{D}+\lambda^{2}-\bar{s}^{2}\right) F(t)\right) d t .
$$


Let $0<\varepsilon<R$. Then performing integration-by-parts and using the fact that the operators $\mathscr{Y}, \mathscr{S}^{\prime}$ and $\tau\left(\Omega_{M_{0}}\right)$ are self-adjoint, we obtain

$$
\begin{aligned}
\int_{\varepsilon}^{R} \varrho(t)\left(\boldsymbol{\delta}_{j, s} \phi_{s}\left(a_{t}\right) \mid\left(\mathscr{D}+\lambda^{2}-\bar{s}^{2}\right) F(t)\right) d t \\
\quad=-\Phi(R)+\Phi(\varepsilon)+\int_{\varepsilon}^{R} \varrho(t)\left(\left(\mathscr{D}+\lambda^{2}-s^{2}\right) \delta_{j, s} \phi_{s}\left(a_{t}\right) \mid F(t)\right) d t
\end{aligned}
$$

with

$$
\begin{aligned}
\Phi(t)=\varrho(t)\left(\boldsymbol{\delta}_{j, s} \phi_{s}\left(a_{t}\right) \mid F^{\prime}(t)\right)-\varrho^{\prime}(t)\left(\boldsymbol{\delta}_{j, s} \phi_{s}\left(a_{t}\right) \mid F(t)\right) \\
-\varrho(t)\left(\frac{d}{d t} \boldsymbol{\delta}_{j, s} \phi_{s}\left(a_{t}\right) \mid F(t)\right) \\
+\varrho(t)((2 n-2 r+1) \tanh t+(2 r-1) \operatorname{coth} t)\left(\boldsymbol{\delta}_{j, s} \phi_{s}\left(a_{t}\right) \mid F(t)\right) .
\end{aligned}
$$

Now we use (40). Since the function $\mathrm{a}_{\alpha}(s)$ appearing there is an even polynomial function of degree no more than $2 \alpha$, we have

$$
\boldsymbol{\delta}_{j, s} \phi_{s}\left(a_{t}\right)=\frac{\mathrm{w}_{\mu-j}}{z^{\mu-j}}(1+O(s ; z, z \log z))
$$

with $\mathrm{w}_{\alpha}=\left(\boldsymbol{\delta}_{\alpha, s} \mathrm{a}_{\alpha}\right)(0)$. Using this, we easily see that

$$
\Phi(t)=4 c_{r}(\mu-j)\left(\mathrm{w}_{\mu-j} \mid F(0)\right) z^{j}(1+O(s ; z, z \log z))
$$

to conclude that

$$
\lim _{\varepsilon \rightarrow+0} \Phi(\varepsilon)=4 c_{r} \mu \delta_{0 j}(\mathrm{w} \mid F(0))
$$

with $\delta_{0 j}$ the Kronecker delta. To compute the limit of $\Phi(R)$ as $R$ goes to infinity, we use (41) and Lemma 3.1. After a computation we obtain

$$
\lim _{R \rightarrow+\infty} \Phi(R)=0
$$

Here we need the inequality $\operatorname{Re} s>n$ which follows from $\operatorname{Re} s>3 n-2 r$. A simple computation (or [Gon and Tsuzuki 2002, Claim 3.1.6, p. 240]) shows the first equality of

$$
\begin{aligned}
\left(\mathscr{D}+\lambda^{2}-s^{2}\right) \delta_{j, s} \phi_{s}\left(a_{t}\right) & =\boldsymbol{\delta}_{j, s}\left(\mathscr{D}+\lambda^{2}-s^{2}\right) \phi_{s}\left(a_{t}\right)+\boldsymbol{\delta}_{j-1, s} \phi_{s}\left(a_{t}\right) \\
& =\boldsymbol{\delta}_{j-1, s} \phi_{s}\left(a_{t}\right),
\end{aligned}
$$

and the differential equation of (22) gives the second equality. Putting together equations (51)-(55), we obtain the result. 


\section{Spectral expansion}

In this section we investigate the spectral expansion of the functions $\boldsymbol{\delta}_{j, s} \tilde{G}_{s}$ to obtain a meromorphic continuation of the current-valued function $s \mapsto G_{s}$, which is already holomorphic on the half plane $\operatorname{Re} s>n$.

Spectral expansion. In order to describe the spectral decomposition of the function $\boldsymbol{\delta}_{\mu, s} \tilde{G}_{s}$, we need some preparations.

For positive $q$, let $\mathscr{L}_{\Gamma}^{q}(\tau)$ denote the Banach space of all measurable functions $f: G \rightarrow \bigwedge \mathfrak{p}_{\mathbb{C}}^{*}$ such that for all $\gamma \in \Gamma$ and $k \in K, f(\gamma g k)=\tau(k)^{-1} f(g)$ and $\int_{\Gamma \backslash G}\|f(g)\|^{q} d \dot{g}<\infty$. For $0 \leqslant d \leqslant n$, let $\mathscr{L}_{\Gamma}^{q}(\tau)^{(d)}$ denote the subspace of those functions $f \in \mathscr{L}_{\Gamma}^{q}(\tau)$ with values in $\bigwedge^{d, d} \mathfrak{p}_{\mathbb{C}}^{*}$. The inner product of two functions $f_{1}$ and $f_{2}$ in $\mathscr{L}_{\Gamma}^{2}(\tau)$ is given as

$$
\left\langle f_{1} \mid f_{2}\right\rangle=\int_{\Gamma \backslash G}\left(f_{1}(g) \mid f_{2}(g)\right) d \dot{g} .
$$

For each $0 \leqslant d \leqslant n$, let $\left\{\lambda_{n}^{(d)}\right\}_{n \in \mathbb{N}}$ be the increasing sequence of the eigenvalues of the bidegree $(d, d)$-part of the Laplacian $\widetilde{\triangle}=-R_{\Omega}$ such that each eigenvalue occurs with its multiplicity. Choose an orthonormal system $\left\{\tilde{\alpha}_{n}^{(d)}\right\}_{n \in \mathbb{N}}$ in $\mathscr{L}_{\Gamma}^{2}(\tau)^{(d)}$ such that $\widetilde{\triangle} \tilde{\alpha}_{n}^{(d)}=\lambda_{n}^{(d)} \tilde{\alpha}_{n}^{(d)}$ for each $n$. From now on we assume $\Gamma$ is not cocompact. Recall the parabolic subgroups $P^{i}$ used to construct the Siegel domains $\mathfrak{S}^{i}$ (page 338). Let $P^{i}=M_{0}^{i} T^{i} N^{i}$ be its Langlands decomposition with $M_{0}^{i}:=Z_{K}\left(T^{i}\right)$. For each $i$ let $\Gamma_{P^{i}}=\Gamma \cap P^{i}$ and $\Gamma_{M_{0}^{i}}=M_{0}^{i} \cap\left(\Gamma_{P^{i}} N^{i}\right)$. Then $\Gamma_{M_{0}^{i}}$ is a finite group which is trivial if $\Gamma$ is torsion free.

For a vector $\mathrm{u} \in \mathrm{V}_{i}^{(d)}:=\left(\bigwedge^{d, d} \mathfrak{p}_{\mathbb{C}}^{*}\right)^{\Gamma_{M_{0}^{i}}}$ and a complex number $s$, define the function $\varphi_{s}^{i}(\mathrm{u} ; g)$ on $G$ using the Iwasawa decomposition $G=N^{i} T^{i} K$ by

$$
\varphi_{s}^{i}\left(\mathrm{u} ; n_{i} \underline{t}_{i} k\right)=t^{s+n} \tau(k)^{-1} \mathrm{u},
$$

for $n_{i} \in N^{i}, t>0, k \in K$. The Eisenstein series associated with $\mathrm{u}$ is defined by the absolutely convergent infinite series

$$
\mathrm{E}^{i}(s ; \mathrm{u} ; g)=\sum_{\gamma \in \Gamma_{p^{i}} \backslash \Gamma} \varphi_{s}^{i}(\mathrm{u} ; \gamma g), \quad g \in G,
$$

if $\operatorname{Re} s>n$. There exists a family of linear maps $\mathrm{E}^{i}(s)$ from $\mathrm{V}_{i}^{(d)}$ to the space of automorphic forms on $\Gamma \backslash G$, which depends meromorphically on $s \in \mathbb{C}$ and is holomorphic on the imaginary axis, such that $\left(\mathrm{E}^{i}(s)(\mathrm{u})\right)(g)=\mathrm{E}^{i}(s ; \mathrm{u} ; g)$ coincides with (56) when $\operatorname{Re} s>n$. If $\mathrm{u} \in \mathrm{V}_{i}^{(d)}$ is an eigenvector of the Casimir operator $\Omega_{M_{0}^{i}}$ of $M_{0}^{i}$ with eigenvalue $c \in \mathbb{C}$, then $R_{\Omega} \mathrm{E}(s ; \mathrm{u})=\left(s^{2}-n^{2}+c\right) \mathrm{E}^{i}(s ; \mathrm{u})$ for any $s \in \mathbb{C}$ where $\mathrm{E}^{i}(s)$ is regular. It is also known that there exists a meromorphic family of operators $\left\{\mathrm{c}_{j}^{i}(s)\right\}$ with $\mathrm{c}_{j}^{i}(s) \in \operatorname{Hom}_{\mathbb{C}}\left(\mathrm{V}_{i}^{(d)}, \mathrm{V}_{j}^{(d)}\right)$ satisfying the functional equations 


$$
\begin{gathered}
\mathrm{E}^{i}(-s)=\sum_{j=1}^{h} \mathrm{E}^{j}(s) \circ \mathrm{c}_{j}^{i}(s), \quad 1 \leqslant i \leqslant h, \\
\sum_{j=1}^{h} \mathrm{c}_{j}^{i}(-\bar{s})^{*} \circ \mathrm{c}_{j}^{i}(s)=\operatorname{Id}_{\mathrm{V}_{i}^{(d)}}, \quad 1 \leqslant i \leqslant h .
\end{gathered}
$$

Lemma 5.1. For $0 \leqslant p \leqslant d$ and $\varepsilon \in\{0,1\}$, let $\mathrm{W}_{i}^{(d)}(p ; \varepsilon)$ be the eigenspace of $\tau\left(\Omega_{M_{0}^{i}}\right)$ on $\mathrm{V}_{i}^{(d)}$ corresponding to the eigenvalue $(2 p-\varepsilon)(2 n-2 p+\varepsilon)$. Then we have the orthogonal decomposition

$$
\mathrm{V}_{i}^{(d)}=\bigoplus_{p=0}^{d} \bigoplus_{\varepsilon \in\{0,1\}} \mathrm{W}_{i}^{(d)}(p ; \varepsilon) .
$$

Proof. First we recall the construction of invariant tensors given in Proposition 1.2. For each $p, q$, let $E_{p, q}$ be the $M_{0}$-module defined in the proof of that proposition and $\mathscr{T}^{p, q}$ be the $M_{0}$-homomorphism constructed there. For convenience we set $E_{p, q}=\{0\}$ when $p$ or $q$ is negative. Choose a parabolic subgroup $P_{0}$ of $G$ with Levi subgroup $M_{0} A$. Then we can find an element $\tilde{k}_{i} \in K$ such that $P^{i}=\tilde{k}_{i} P_{0} \tilde{k}_{i}^{-1}$, $M_{0}^{i}=\tilde{k}_{i} M_{0} \tilde{k}_{i}^{-1}$, and $T^{i}=\tilde{k}_{i} A \tilde{k}_{i}^{-1}$. For $p \in \mathbb{N}$, put $p(+):=p$ and $p(-):=p-1$. For $\varepsilon, \varepsilon^{\prime} \in\{+,-\}$, set $E_{p, \varepsilon \varepsilon^{\prime}}=\mathscr{T}^{p, p}\left(E_{p(\varepsilon), p\left(\varepsilon^{\prime}\right)}\right)$; then $E_{p, \varepsilon, \varepsilon^{\prime}}^{i}:=\tau\left(\tilde{k}_{i}\right)\left(E_{p, \varepsilon \varepsilon^{\prime}}\right)$ is an irreducible sub $M_{0}^{i}$-module of $F_{p, p}$. For each $0 \leqslant p<2 n$, the space $F_{p, p}$, when considered as an $M_{0}^{i}$-module, is decomposed to the orthogonal direct sum of four subspaces $E_{p, \varepsilon \varepsilon^{\prime}}^{i}$ with $\varepsilon, \varepsilon^{\prime} \in\{+,-\}$. The operator $\tau\left(\Omega_{M_{0}^{i}}\right)$ acts on $E_{p, \varepsilon \varepsilon^{\prime}}^{i}$ by the scalar $\left(p(\varepsilon)+p\left(\varepsilon^{\prime}\right)\right)\left(2 n-p(\varepsilon)-p\left(\varepsilon^{\prime}\right)\right)$. Now put

$$
\begin{aligned}
& \mathrm{W}_{i}^{(d)}(p ; 0)=L^{d-p}\left(E_{p,++}^{i}\right)^{\Gamma_{M_{0}^{i}}} \oplus L^{d-p-1}\left(E_{p+1,--}^{i}\right)^{\Gamma_{M_{0}^{i}}}, \\
& \mathrm{~W}_{i}^{(d)}(p ; 1)=L^{d-p}\left(E_{p,+-}^{i}\right)^{\Gamma_{M_{0}^{i}}} \oplus L^{d-p}\left(E_{p,-+}^{i}\right)^{\Gamma_{M_{0}^{i}}} .
\end{aligned}
$$

Then the operator $\tau\left(\Omega_{M_{0}^{i}}\right)$ acts on $\mathrm{W}_{i}^{(d)}(p ; \varepsilon)$ for $0 \leqslant p \leqslant d$ and $\varepsilon \in\{0,1\}$ as the scalar $(2 p-\varepsilon)(2 n-2 p+\varepsilon)$. By the $K$-decomposition $(27)$, the space $\mathrm{V}_{i}^{(d)}$ is decomposed as the orthogonal direct sum of those subspaces $\mathrm{W}_{i}^{(d)}(p ; \varepsilon)$.

Lemma 5.2. (a) The eigenfunctions $\tilde{\alpha}_{m}^{(d)}$ lie in the space $\mathfrak{M}_{\delta}$ for any $\delta \in\left(2 r n^{-1}, 1\right)$. For each $0 \leqslant j \leqslant \mu$, we have

$$
\int_{\Gamma \backslash G}\left(\boldsymbol{\delta}_{j, s} \tilde{G}_{s}(g) \mid \tilde{\alpha}_{m}^{(\mu)}(g)\right) d \dot{g}=\frac{4\left(\mathrm{w} \mid \mathscr{F}_{H}\left(\tilde{\alpha}_{m}^{(\mu)} ; e\right)\right)}{\mu !\left(\lambda^{2}-\lambda_{m}^{(\mu)}-s^{2}\right)^{j+1}} .
$$

(b) Let $U$ be a compact subset on which the function $\mathrm{E}^{i}(s)$ is holomorphic. Then for all $\mathrm{u} \in \mathrm{V}_{i}^{(d)}$, the image $\mathrm{E}^{i}(s)(\mathrm{u})$ is in $\mathfrak{M}_{\delta}$ for any $\delta \in\left(2 r n^{-1}, 1\right)$ such that $\sup _{s \in U}|\operatorname{Re} s|<1-\delta$. If $\mathrm{u} \in \mathrm{V}_{i}^{(\mu)}$ satisfies $\tau\left(\Omega_{M_{0}^{i}}\right) \mathrm{u}=c \mathrm{u}$, we have, for each 
$0 \leqslant j \leqslant \mu$,

$$
\int_{\Gamma \backslash G}\left(\delta_{j, s} \tilde{G}_{s}(g) \mid \mathrm{E}^{i}(v ; \mathrm{u}, ; g)\right) d \dot{g}=\frac{4\left(\mathrm{w} \mid \mathscr{F}_{H}\left(\mathrm{E}^{i}(v ; \mathrm{u}) ; e\right)\right)}{\mu !\left(\lambda^{2}-n^{2}+c-s^{2}+v^{2}\right)^{j+1}}
$$

for $s \in \mathbb{C}$ with $|\operatorname{Re} s|<1-2 r n^{-1}$.

Proof. The estimation in [Oda and Tsuzuki 2003, Lemma 3.3.1], which implies the first assertion of (a), is valid for our $\tilde{\alpha}_{m}^{(d)}$ without modification. The first assertion of (b) follows from [Miatello and Wallach 1989, Lemma A.2.2]. The argument to prove (59) and (60) by Theorem 4.5 is the same as that in the proof of [Oda and Tsuzuki 2003, Proposition 6.2.2].

For each index $(d, i, p, \varepsilon)$, fix an orthonormal basis $\mathscr{B}_{i}^{(d)}(p ; \varepsilon)$ of the space $\mathrm{W}_{i}^{(d)}(p ; \varepsilon)$.

Theorem 5.3. Let $\operatorname{Re} s>3 n-2 r$. Then there exists $\varepsilon>0$ such that the function $\boldsymbol{\delta}_{\mu, s} \tilde{G}_{s}(g)$ belongs to the space $\mathscr{L}_{\Gamma}^{2+\varepsilon}(\tau)^{(\mu)}$. The spectral expansion of $\boldsymbol{\delta}_{\mu, s} \tilde{G}_{s}$ is

$$
\boldsymbol{\delta}_{\mu, s} \tilde{G}_{s}=\mathscr{\varphi}_{\mathrm{dis}}(s)+\sum_{p=0}^{\mu} \sum_{\varepsilon \in\{0,1\}} \mathscr{G}_{\mathrm{c}}^{(p, \varepsilon)}(s)
$$

with

$$
\begin{aligned}
& \mathscr{G}_{\mathrm{dis}}(s)=\sum_{m=0}^{\infty} \frac{4\left(\mathrm{w} \mid \mathscr{F}_{H}\left(\tilde{\alpha}_{m}^{(\mu)} ; e\right)\right)}{\mu !\left(\lambda^{2}-\lambda_{m}^{(\mu)}-s^{2}\right)^{r}} \tilde{\alpha}_{m}^{(\mu)}, \\
& \mathscr{G}_{\mathrm{c}}^{(p, \varepsilon)}(s)=\frac{1}{4 \pi \sqrt{-1}} \\
& \times \int_{\sqrt{-1} \mathbb{R}} \sum_{i=1}^{h} \sum_{\mathrm{u} \in \mathscr{B}_{i}^{(\mu)}(p ; \varepsilon)} \frac{4\left(\mathrm{w} \mid \mathscr{F}_{H}\left(\mathrm{E}^{i}(\zeta ; \mathrm{u}) ; e\right)\right)}{\mu !\left(\lambda^{2}-(n-2 p+\varepsilon)^{2}-s^{2}+\zeta^{2}\right)^{r}} \mathrm{E}^{i}(\zeta ; \mathrm{u}) d \zeta,
\end{aligned}
$$

where the summations in the right-hand side of this formula are convergent in $\mathscr{L}_{\Gamma}^{2}(\tau)^{(\mu)}$.

Proof. Since the differential operator $\boldsymbol{\delta}_{\mu, s}$ annihilates the even polynomial functions $a_{j}(s)(0 \leqslant j \leqslant \mu-1)$ appearing in (40), the behavior of $\delta_{\mu, s} \phi_{s}\left(a_{t}\right)$ near $t=0$ is given as $\boldsymbol{\delta}_{\mu, s} \phi_{s}\left(a_{t}\right)=O\left(s ; 1, \log z, z^{\mu+2}(\log z)^{2}\right)$. By (41), the function $\delta_{\mu, s} \phi_{s}\left(a_{t}\right)$ has an exponential decay as $t \rightarrow+\infty$ when $\operatorname{Re} s>n$. Hence we can argue in exactly the same way as [Oda and Tsuzuki 2003, $\S 5.2,5.3]$ to obtain the estimate $\boldsymbol{\delta}_{\mu, s} \tilde{G}_{s} \in \mathscr{L}_{\Gamma}^{2+\varepsilon}(\tau)^{(\mu)}$ with some $\varepsilon>0$. (In this reference, the results in Section 4, on which the arguments in $\S \S 5.2$ and 5.3 rely, remain valid for our $(G, H, \Gamma)$ without modification.) Once we establish the $L^{2+\varepsilon}$-estimate of $\boldsymbol{\delta}_{\mu, s} \tilde{G}_{s}$, we can work out the spectral expansion of $\boldsymbol{\delta}_{\mu, s} \tilde{\boldsymbol{G}}_{s}$ using (59) and (60) by the same argument as in [Oda and Tsuzuki 2003, 6.2]. 


\section{Some properties of Eisenstein period.}

Proposition 5.4. For $1 \leqslant i \leqslant h$ and $\mathrm{u} \in \mathrm{V}_{i}^{(d)}$, there exists a unique $\bigwedge^{d, d} \mathfrak{p}_{\mathbb{C}}^{*}$-valued meromorphic function $\mathscr{P}_{H}^{i}(s ; \mathrm{u})$ on $\mathbb{C}$ which is regular and has the value given by the absolutely convergent integral $\mathscr{F}_{H}\left(\mathrm{E}^{i}(s ; \mathrm{u}) ; e\right)$ at any regular point $s \in \mathbb{C}$ of $\mathrm{E}^{i}(s ; \mathrm{u})$ in $|\operatorname{Re} s|<1-2 r n^{-1}$.

Proof. This can be proved by the same argument as in [Oda and Tsuzuki 2003, Proposition 6.1.1].

Proposition 5.5. Let $1 \leqslant i \leqslant h$ and $1 \leqslant p \leqslant d$. Then for any $\mathrm{u} \in \mathrm{W}_{i}^{(d)}(p ; 1)$, we have $\mathscr{P}_{H}^{i}(s ; \mathrm{u})=0$ identically.

Proof. We freely use the notation introduced in the proof of Lemma 5.1. For any unitary representation $(E, \sigma)$ of $M_{0}$ and $s \in \mathbb{C}$, let $\operatorname{Ind}_{P_{0}}^{G}(E ; s)$ be the Fréchet space of all $C^{\infty}$-functions $f: G \rightarrow E$ satisfying the relation $f\left(m a_{t} n g\right)=e^{(n+s) t} \sigma(m) f(g)$ for any $m \in M_{0}, a_{t} \in A$ and $n \in N$. The group $G$ acts by the right translation on the space $\operatorname{Ind}_{P_{0}}^{G}(E ; s)$ smoothly. For $\varepsilon, \varepsilon^{\prime} \in\{+,-\}, 1 \leqslant i \leqslant h, 0 \leqslant p \leqslant d$, put

$$
u_{p, \varepsilon \varepsilon^{\prime}}^{i}:=\operatorname{Hom}_{\Gamma_{M_{0}^{i}}}\left(E_{p, \varepsilon \varepsilon^{\prime}}^{i}, \mathbb{C}\right) .
$$

Then by the theory of Eisenstein series there exists a meromorphic family of continuous $G$-homomorphisms

$$
\mathrm{E}_{p, \varepsilon \varepsilon^{\prime}}^{i}(s): \operatorname{Ind}_{P_{0}}^{G}\left(E_{p, \varepsilon \varepsilon^{\prime}} ; s\right) \otimes \mathcal{U}_{p, \varepsilon \varepsilon^{\prime}}^{i} \rightarrow \mathscr{A}(\Gamma \backslash G), \quad s \in \mathbb{C},
$$

which is given by the absolutely convergent sum

$$
\left(\mathrm{E}_{p, \varepsilon \varepsilon^{\prime}}^{i}(s)(f \otimes \check{u})\right)(g)=\sum_{\gamma \in \Gamma_{P} \backslash \Gamma \Gamma} \check{u}\left(\tau\left(\tilde{k}_{i}\right) f\left(\tilde{k}_{i}^{-1} \gamma g\right)\right), \quad g \in G,
$$

for $f \in \operatorname{Ind}_{P_{0}}^{G}\left(E_{p, \varepsilon \varepsilon^{\prime}} ; s\right)$ and $\check{u} \in \mathcal{U}_{p, \varepsilon \varepsilon^{\prime}}^{i}$ when Re $s>n$. Here $\mathscr{A}(\Gamma \backslash G)$ denotes the space of (not necessarily $K$-finite) automorphic forms on $G$. By slightly extending the argument in the proof of [Oda and Tsuzuki 2003, Proposition 6.1.1], we can show that the integral $\Phi_{p, \varepsilon \mathcal{E}^{\prime}}^{i}(s)(f \otimes \check{u}):=\mathscr{F}_{H}\left(\mathrm{E}_{p, \varepsilon \mathcal{E}^{\prime}}^{i}(s)(f \otimes \check{u}) ; e\right)$ is convergent in some neighborhood of the imaginary axis and the function $\Phi_{p, \varepsilon \varepsilon^{\prime}}^{i}(s)$ has a meromorphic continuation to $\mathbb{C}$. The linear map

$$
\Phi_{p, \varepsilon \varepsilon^{\prime}}^{i}(s): \operatorname{Ind}_{P_{0}}^{G}\left(E_{p, \varepsilon \varepsilon^{\prime}} ; s\right) \otimes \mathcal{U}_{p, \varepsilon \varepsilon^{\prime}}^{i} \rightarrow \mathbb{C}
$$

is continuous and $H$-invariant, that is, $\Phi_{p, \varepsilon \varepsilon^{\prime}}^{i}(s)$ is an $H$-spherical distribution. The maps $\mathrm{E}_{p, \varepsilon \varepsilon^{\prime}}^{i}(s)$ and $\Phi_{p, \varepsilon \varepsilon^{\prime}}^{i}(s)$ naturally induce the linear maps

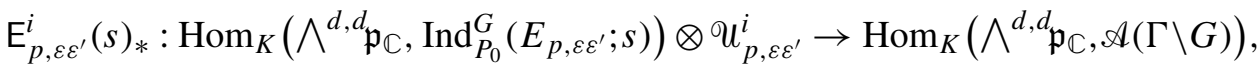

$$
\begin{aligned}
& \Phi_{p, \varepsilon \mathcal{E}^{\prime}}^{i}(s)_{*}: \operatorname{Hom}_{K}\left(\bigwedge^{d, d} \mathfrak{p}_{\mathbb{C}}, \operatorname{Ind}_{P_{0}}^{G}\left(E_{p, \varepsilon \varepsilon^{\prime}} ; s\right)\right) \otimes u_{p, \varepsilon \mathcal{E}^{\prime}}^{i} \rightarrow \bigwedge^{d, d} \mathfrak{p}_{\mathbb{C}}^{*} .
\end{aligned}
$$


On the other hand, we define the linear map

$$
J_{p, \varepsilon \varepsilon^{\prime}}^{i}: \operatorname{Hom}_{K}\left(\bigwedge^{d, d} \mathfrak{p}_{\mathbb{C}}, \operatorname{Ind}_{P_{0}}^{G}\left(E_{p, \varepsilon \varepsilon^{\prime}} ; s\right)\right) \otimes \mathcal{U}_{p, \varepsilon \varepsilon^{\prime}}^{i} \rightarrow \mathrm{V}_{i}^{(d)}
$$

by composing the maps

$$
\begin{aligned}
& \operatorname{Hom}_{K}\left(\bigwedge^{d, d} \mathfrak{p}_{\mathbb{C}}, \operatorname{Ind}_{P_{0}}^{G}\left(E_{p, \varepsilon \varepsilon^{\prime}} ; s\right)\right) \otimes u_{p, \varepsilon \varepsilon^{\prime}}^{i} \\
& \quad \stackrel{J_{1}}{\rightarrow} \operatorname{Hom}_{K}\left(\bigwedge^{d, d} \mathfrak{p}_{\mathbb{C}}, \operatorname{Ind}_{M_{0}}^{K}\left(E_{p, \varepsilon \varepsilon^{\prime}} \otimes u_{p, \varepsilon \varepsilon^{\prime}}^{i}\right)\right) \\
& \quad \stackrel{J_{2}}{\rightarrow} \operatorname{Hom}_{K}\left(\bigwedge^{d, d} \mathfrak{p}_{\mathbb{C}}, \operatorname{Ind}_{M_{0}^{i}}^{K}\left(E_{p, \varepsilon \varepsilon^{\prime}}^{i} \otimes u_{p, \varepsilon \varepsilon^{\prime}}^{i}\right)\right) \stackrel{J_{3}}{\rightarrow} \operatorname{Hom}_{M_{0}^{i}}\left(\bigwedge^{d, d} \mathfrak{p}_{\mathbb{C}}, E_{p, \varepsilon \varepsilon^{\prime}} \otimes u_{p, \varepsilon \varepsilon^{\prime}}^{i}\right) \\
& \stackrel{J_{4}}{\rightarrow} \operatorname{Hom}_{M_{0}^{i}}\left(\bigwedge^{d, d} \mathfrak{p}_{\mathbb{C}}, \operatorname{Ind}_{\Gamma_{M_{0}^{i}}^{M^{i}}}^{M_{\Gamma^{i}}}\left(1_{\Gamma_{0}^{i}}\right)\right) \stackrel{J_{5}}{\rightarrow} \operatorname{Hom}_{\Gamma_{M_{0}^{i}}}\left(\bigwedge^{d, d} \mathfrak{p}_{\mathbb{C}}, 1_{\Gamma_{M_{0}^{i}}} \cong \mathrm{V}_{i}^{(d)},\right.
\end{aligned}
$$

where $J_{1}$ is the isomorphism induced by the natural identification of the $K$-modules $\operatorname{Ind}_{P_{0}}^{G}\left(E_{p, \varepsilon \varepsilon^{\prime}} ; s\right) \otimes \mathcal{U}_{p, \varepsilon \varepsilon^{\prime}}^{i} \cong \operatorname{Ind}_{M_{0}}^{K}\left(E_{p, \varepsilon \varepsilon^{\prime}} \otimes u_{p, \varepsilon \varepsilon^{\prime}}^{i}\right)$, the map $J_{2}$ is induced by the $K$-isomorphism $\operatorname{Ind}_{M_{0}}^{K}\left(E_{p, \varepsilon \varepsilon^{\prime}} \cong \operatorname{Ind}_{M_{0}^{i}}^{K}\left(E_{p, \varepsilon \varepsilon^{\prime}}^{i}\right)\right.$ that assigns the function

$$
k \mapsto \tau\left(\tilde{k}_{i}\right) f\left(\tilde{k}_{i}^{-1} k\right) \in \operatorname{Ind}_{M_{0}^{i}}^{K}\left(E_{p, \varepsilon \varepsilon^{\prime}}^{i}\right) \quad \text { to } \quad f(k) \in \operatorname{Ind}_{M_{0}}^{K}\left(E_{p, \varepsilon \varepsilon^{\prime}}\right),
$$

$J_{3}$ and $J_{5}$ are the isomorphisms giving the Frobenius reciprocity, and $J_{4}$ is the inclusion induced by the map $E_{p, \varepsilon \varepsilon^{\prime}}^{i} \otimes u_{p, \varepsilon \varepsilon^{\prime}}^{i} \hookrightarrow C^{\infty}\left(\Gamma_{M_{0}^{i}}^{\backslash} M_{0}^{i}\right)$ which identifies the tensor $\mathrm{u} \otimes \check{u} \in E_{p, \varepsilon \varepsilon^{\prime}}^{i} \otimes \mathcal{U}_{p, \varepsilon \varepsilon^{\prime}}^{i}$ with the function $m \mapsto\langle\check{u}, \tau(m) \mathrm{u}\rangle$ on $\Gamma_{M_{0}^{i}}^{\backslash} \backslash M_{0}^{i}$. The map $J_{p, \varepsilon \varepsilon^{\prime}}^{i}$ is injective and has the defining formula

$$
\left\langle J_{p, \varepsilon \varepsilon^{\prime}}^{i}(\alpha \otimes \check{u}), \xi\right\rangle=\left\langle\check{u}, \tau\left(\tilde{k}_{i}\right)\left(\alpha(\xi)\left(\tilde{k}_{i}^{-1}\right)\right)\right\rangle
$$

for $\xi \in \bigwedge^{d, d} \mathfrak{p}_{\mathbb{C}}, \alpha \in \operatorname{Hom}_{K}\left(\bigwedge^{d, d} \mathfrak{p}_{\mathbb{C}}, \operatorname{Ind}_{P_{0}}^{G}\left(E_{p, \varepsilon \varepsilon^{\prime}} ; s\right)\right)$, and $\check{u} \in u_{p, \varepsilon \varepsilon^{\prime}}^{i}$. From the definition of $J_{p, \varepsilon \varepsilon^{\prime}}^{i}$ and $(58)$, we get $\mathrm{W}_{i}^{(d)}(p ; 1)=\operatorname{Im} J_{p,+-}^{i} \oplus \operatorname{Im} J_{p,-+}^{i}$. The formula

$$
\mathrm{E}^{i}(s ; \mathrm{u})=\left(\mathrm{E}_{p,+-}^{i}(s)_{*} \oplus \mathrm{E}_{p,-+}^{i}(s)_{*}\right) \circ\left(J_{p,+-}^{i} \oplus J_{p,-+}^{i}\right)^{-1}(\mathrm{u}), \quad \mathrm{u} \in \mathrm{W}_{i}^{(d)}(p ; 1),
$$

which follows directly from the definitions when $\operatorname{Re} s>n$, remains valid as an identity of meromorphic functions on $\mathbb{C}$. When $|\operatorname{Re} s|$ is sufficiently small, the integration on $\Gamma_{H} \backslash H$ of this formula yields yet another formula: for $\mathrm{u} \in \mathrm{W}_{i}^{(d)}(p ; 1)$,

$$
\mathscr{F}_{H}\left(\mathrm{E}^{i}(s ; \mathrm{u}) ; e\right)=\left(\Phi_{p,+-}^{i}(s)_{*} \oplus \Phi_{p,-+}^{i}(s)_{*}\right) \circ\left(J_{p,+-}^{i} \oplus J_{p,-+}^{i}\right)^{-1}(\mathrm{u}) .
$$

By the general theory, there exists an open dense set $U$ such that for $s \in U$ the space $C^{-\infty} \operatorname{Ind}_{P_{0}}^{G}\left(E_{p, q} ; s\right)^{H}$ of $H$-spherical distributions is isomorphic to $E_{p, q}^{M_{0} \cap H}=E_{p, q}^{M}$ [Heckman and Schlichtkrull 1994, Theorem 6.4, p. 151], which is zero when $p \neq q$ by the Claim in the proof of Proposition 1.2. Hence $\Phi_{p,+-}^{i}(s)=0$ and $\Phi_{p,-+}^{i}(s)=0$ for $s \in U$. This, combined with (62), implies $\mathscr{S}_{H}\left(\mathrm{E}^{i}(s ; \mathrm{u}) ; e\right)=0$ for generic $s$. By analytic continuation we obtain $\mathscr{P}_{H}^{i}(s ; \mathrm{u})=0$ identically. 
Lemma 5.6. For each $0 \leqslant p \leqslant \mu$, put

$$
\tilde{\mathscr{E}}_{p}^{(\mu)}(v ; g)=\frac{4}{\mu !} \sum_{i=1}^{h} \sum_{\mathrm{u} \in \mathscr{B}_{i}^{(\mu)}(p ; 0)}\left(\mathrm{w} \mid \mathscr{P}_{H}^{i}(-\bar{v} ; \mathrm{u})\right) \mathrm{E}^{i}(v ; \mathrm{u} ; g), \quad g \in G, v \in \mathbb{C} .
$$

Then $\tilde{\mathscr{E}}_{p}^{(\mu)}(v ; g)$ is independent of the choice of the orthonormal basis $\mathscr{B}_{i}^{(\mu)}(p ; 0)$ and satisfies the functional equation $\tilde{\mathscr{E}}_{p}^{(\mu)}(-s ; g)=\tilde{\mathscr{E}}_{p}^{(\mu)}(s ; g)$.

Proof. The independence of the basis $\mathscr{B}_{i}^{(\mu)}(p ; 0)$ is clear to see. The functional equation follows from (57).

Meromorphic continuation and functional equations. Let $\mathscr{K}_{\Gamma}(\tau)$ be the space of $C^{\infty}$-functions $\tilde{\beta}: G \rightarrow \bigwedge \mathfrak{p}_{\mathbb{C}}^{*}$ with compact support modulo $\Gamma$ such that $\tilde{\beta}(\gamma g k)=$ $\tau(k)^{-1} \tilde{\beta}(g)$ for all $\gamma \in \Gamma$ and $k \in K$.

Theorem 5.7. Let $L_{1}$ be the interval on the imaginary axis defined by (25). Let $0 \leqslant j \leqslant \mu$. Then for each $\tilde{\beta} \in \mathcal{K}_{\Gamma}(\tau)$, on $\operatorname{Re} s>n$ the holomorphic function $s \mapsto \mathscr{G}_{j}(s, \tilde{\beta}):=\left\langle\boldsymbol{\delta}_{j, s} \tilde{G}_{s} \mid \tilde{\beta}\right\rangle$ has a meromorphic continuation to the domain $\mathbb{C}-L_{1}$. A point $s_{0} \in \mathbb{C}-L_{1}$ with $\operatorname{Re} s_{0} \geqslant 0$ is a pole of the meromorphic function $\mathscr{G}_{j}(s, \beta)$ if and only if there exists an $m \in \mathbb{N}$ such that $\left(\mathrm{w} \mid \mathscr{F}_{H}\left(\tilde{\alpha}_{m}^{(\mu)} ; e\right)\right) \neq 0,\left\langle\tilde{\alpha}_{m}^{(\mu)} \mid \tilde{\beta}\right\rangle \neq 0$ and $s_{0}^{2}-\lambda^{2}=-\lambda_{m}^{(\mu)}$. In this case, the function

$$
\mathscr{G}_{j}(s, \beta)-\sum_{\substack{m \in \mathbb{N} \\ \lambda_{m}^{(\mu)}=\lambda^{2}-s_{0}^{2}}} \frac{4\left(\mathrm{w} \mid \mathscr{F}_{H}\left(\tilde{\alpha}_{m}^{(\mu)} ; e\right)\right)\left\langle\tilde{\alpha}_{m}^{(\mu)} \mid \tilde{\beta}\right\rangle}{\mu !\left(s_{0}^{2}-s^{2}\right)^{j+1}}
$$

is holomorphic at $s=s_{0}$. We have the functional equation

$$
\mathscr{G}_{j}(-s, \tilde{\beta})-\mathscr{G}_{j}(s, \tilde{\beta})=\boldsymbol{\delta}_{j, s}\left(\sum_{p=0}^{\mu} \frac{\left\langle\tilde{\mathscr{E}}_{p}^{(\mu)}\left(v_{s}^{(p+1)}\right) \mid \tilde{\beta}\right\rangle}{2 v_{s}^{(p+1)}}\right) .
$$

Proof. We prove the assertions by downward-induction on $j$. Consider the case of $j=\mu$. By the same argument in [Oda and Tsuzuki 2003, $\S \S 6.2,6.3$ ], we see that the series $\mathscr{G}_{\text {dis }}(s)$ is convergent in $\mathscr{L}_{\Gamma}^{2}(\tau)$ for any $s \in \mathbb{C}$ such that for all $m$, $\lambda_{m}^{(\mu)} \neq \lambda^{2}-s^{2}$ it gives an $\mathscr{L}_{\Gamma}^{2}(\tau)$-valued meromorphic function on $\mathbb{C}$ (namely, for each $s_{0} \in \mathbb{C}$ there exists $a \in \mathbb{Z}$ such that $\left(s-s_{0}\right)^{a} \mathscr{G}_{\mathrm{dis}}(s)$ is holomorphic around $\left.s_{0}\right)$. By Proposition 5.5, $\mathscr{G}_{\mu}(s)-\mathscr{G}_{\mathrm{dis}}(s)$ is the sum over $p$ of

$$
\mathscr{G}_{\mathrm{c}}^{(p, 0)}(s)=\frac{1}{4 \pi \sqrt{-1}} \int_{\sqrt{-1} \mathbb{R}} \frac{\tilde{\mathscr{E}}_{p}^{(\mu)}(\zeta)}{\left(\zeta^{2}-\left(v_{s}^{(p+1)}\right)^{2}\right)^{r}} d \zeta, \quad 0 \leqslant p \leqslant \mu .
$$

Since $\operatorname{Re} v_{s}^{(p+1)}>\operatorname{Re} s$ for $\operatorname{Re} s>0$ by Lemma 3.1, the denominator of the integrand is never zero as long as $\zeta \in \sqrt{-1} \mathbb{R}$ and $\operatorname{Re} s>0$. Hence the same argument 
just cited proves the convergence of the integral $\varphi_{\mathrm{c}}^{(p, 0)}(s)$ in $\mathscr{L}_{\Gamma}^{2}(\tau)$ not only on $\operatorname{Re} s>3 n-2 r$ but also on the broader domain $\operatorname{Re} s>0$, and moreover the integral defines a holomorphic function on $\operatorname{Re} s>0$. Thus a meromorphic continuation of $\mathscr{G}_{\mu}(s, \beta)=\left\langle\mathscr{G}_{\mathrm{dis}}(s) \mid \tilde{\beta}\right\rangle+\sum_{p}\left\langle\mathscr{G}_{\mathrm{c}}^{(p, 0)}(s) \mid \tilde{\beta}\right\rangle$ exists.

The next step is to obtain the analytic continuation of $\mathscr{G}_{\mu}(s, \beta)$ around a point $s_{0}=\sqrt{-1} \sigma_{0} \in \sqrt{-1} \mathbb{R}-L_{1}$. For that purpose, we consider the same problem for each integrals $\left\langle\varphi_{\mathrm{c}}^{(p, 0)}(s) \mid \tilde{\beta}\right\rangle$. Put $\zeta_{0}=v_{s_{0}}^{(p+1)}$. Note that $\zeta_{0} \in \sqrt{-1} \mathbb{R}$. Let $a, b>0$ be arbitrary numbers such that the functions $\left\langle\mathscr{E}_{p}^{(}(\mu)(\zeta) \mid \tilde{\beta}\right\rangle, 0 \leqslant p \leqslant \mu$, are holomorphic on the open rectangle $R_{a, b}\left(\zeta_{0}\right)$ having the vertices $\zeta_{0} \pm a \pm \sqrt{-1} b$. Let $C_{a, b}$ be the path which, as a point set, is a union of $\sqrt{-1} \mathbb{R}-\left[\zeta_{0}-b \sqrt{-1}, \zeta_{0}+b \sqrt{-1}\right]$ and $\partial R_{a, b}\left(\zeta_{0}\right) \cap\{\operatorname{Re} \zeta \geqslant 0\}$, and which rounds the point $\zeta_{0}$ counterclockwise. Let $U_{a, b}\left( \pm s_{0}\right)$ be the inverse image of $R_{a, b}\left(\zeta_{0}\right)$ by the map $s \mapsto v_{s}^{(p+1)}$; thus $U_{a, b}\left( \pm s_{0}\right)$ is an open neighborhood of $\left\{s_{0},-s_{0}\right\}$ in $\mathbb{C}-L_{1}$. For $s \in U_{a, b}\left( \pm s_{0}\right) \cap\{\operatorname{Re} s>0\}$, by the residue theorem,

$$
\begin{aligned}
& =\frac{1}{4 \pi \sqrt{-1}}\left(\int_{C_{a, b}} \frac{\left\langle\tilde{\mathscr{E}}_{p}^{(\mu)}(\zeta) \mid \tilde{\beta}\right\rangle}{\left(\zeta^{2}-\left(v_{s}^{(p+1)}\right)^{2}\right)^{r}} d \zeta-2 \pi \sqrt{-1} \operatorname{Res}_{z=v_{s}^{(p+1)}} \frac{\left\langle\tilde{\mathscr{E}}_{p}^{(\mu)}(\zeta) \mid \tilde{\beta}\right\rangle}{\left(\zeta^{2}-\left(v_{s}^{(p+1)}\right)^{2}\right)^{r}}\right) \\
& =\frac{1}{4 \pi \sqrt{-1}} \int_{C_{a, b}} \frac{\left\langle\tilde{\mathscr{E}}_{p}^{(\mu)}(\zeta) \mid \tilde{\beta}\right\rangle}{\left(\zeta^{2}-\left(v_{s}^{(p+1)}\right)^{2}\right)^{r}} d \zeta-\frac{1}{4} \boldsymbol{\delta}_{\mu, s}\left(\frac{\left\langle\tilde{\mathscr{E}}_{p}^{(\mu)}\left(v_{s}^{(p+1)}\right) \mid \tilde{\beta}\right\rangle}{v_{s}^{(p+1)}}\right) .
\end{aligned}
$$

using Lemma 5.8 to compute the residue. The integral in the first term of this expression is convergent even for $s \in U_{a, b}\left( \pm s_{0}\right)$ and defines a holomorphic function on $U_{a, b}\left( \pm s_{0}\right)$. Since the second term is also holomorphic on $U_{a, b}\left( \pm s_{0}\right)$, we obtain an analytic continuation of $\left\langle\varphi_{c}^{(p, 0)}(s) \mid \tilde{\beta}\right\rangle$ on a neighborhood of $\left\{s_{0},-s_{0}\right\}$. The functional equation (64) for $s \in U_{a, b}\left( \pm s_{0}\right)$ follows if we note that the first term in the right-hand side of the second identity of (65) is invariant under the substitution $s \mapsto-s$ and also note the equation $v_{-s}^{(p+1)}=-v_{s}^{(p+1)}$; see Lemma 3.1. Once the functional equation (64) is established on a small open set of the form $U_{a, b}\left( \pm s_{0}\right)$ with $s_{0} \in \sqrt{-1} \mathbb{R}-L_{1}$, we can use it to obtain a meromorphic continuation by defining the value $\mathscr{G}_{\mu}(s, \beta)$ for $\operatorname{Re} s<0$ in terms of $\mathscr{G}_{\mu}(-s, \beta)$ which is defined above and the terms containing the derivative of $\langle\mathscr{E}(\mu)(s) \mid \tilde{\beta}\rangle$ which is meromorphic on $\mathbb{C}$; see Proposition 5.4. The poles of $\left\langle\mathscr{G}_{\mu}(s) \mid \tilde{\beta}\right\rangle$ on $\operatorname{Re} s \geqslant 0$ stem only from the discrete part $\left\langle\mathscr{G}_{\mathrm{dis}}(s) \mid \tilde{\beta}\right\rangle$, whose series expression (61) itself proves the criterion in the theorem for $s_{0}$ to be a pole, as well as a statement on the behavior around the poles. This completes the proof of Theorem for $j=\mu$.

We prove the theorem for $j$ assuming it holds for $j+1$. Since

$$
\varphi_{j+1}(s, \beta)=(j+1)^{-1} \frac{1}{2 s} \frac{d}{d s} \varphi_{j}(s, \beta)
$$


for $\operatorname{Re} s>n$ by Proposition 4.4, the function $\varphi_{j}(s, \beta)$ should be a primitive function of $2(j+1) s \mathscr{G}_{j+1}(s, \beta)$. Fix a point $z_{0}$ with $\operatorname{Re} z_{0}>n$ so that the value $\mathscr{G}_{j}\left(z_{0}, \beta\right)$ is already defined. For each $s_{0} \in \sqrt{-1} \mathbb{R}-L_{1}$, let $U_{a, b}\left( \pm s_{0}\right)$ be its neighborhood constructed above and put $D_{a, b}\left( \pm s_{0}\right)=U_{a, b}\left( \pm s_{0}\right) \cup\{\operatorname{Re} s>0\}$. We take sufficiently small $a$ and $b$ such that $\left\langle\mathscr{E}_{p}^{(\mu)}\left(v_{s}^{(p+1)}|\tilde{\beta}\rangle\right.\right.$ are all regular on $U_{a, b}\left( \pm s_{0}\right)$. For a path $C_{s}$ connecting $z_{0}$ and $s \in D_{a, b}\left( \pm s_{0}\right)$ inside $D_{a, b}\left( \pm s_{0}\right)$, consider the integral

$$
\tilde{\mathscr{G}}(s)=2(j+1) \int_{C_{s}} \zeta \varphi_{j+1}(\zeta, \beta) d \zeta+\varphi_{j}\left(z_{0}, \beta\right) .
$$

Since the residues of $\varphi_{j+1}(s, \beta)$ at any poles in $\operatorname{Re} s \geqslant 0$ are all zero by inductionassumption and since the poles of $\mathscr{G}_{j+1}(s, \beta)$ in $D_{a, b}\left( \pm s_{0}\right)$ are automatically in $\operatorname{Re} s \geqslant 0$ by the choice of $a, b$, this integral is independent of the choice of the path $C_{s}$ and defines a meromorphic function of $s$ on $D_{a, b}\left( \pm s_{0}\right)$. Since $\tilde{\mathscr{G}}\left(z_{0}\right)=\mathscr{G}_{j}\left(z_{0}, \beta\right)$ and $2(j+1) s \mathscr{G}_{j+1}(s, \beta)=d / d s \tilde{\mathscr{G}}(s)$ for $\operatorname{Re} s>n$, we have $\tilde{\mathscr{G}}(s)=\mathscr{G}_{j}(s)$ at least on $\operatorname{Re} s>n$. Thus the integral expression (66) gives us an analytic continuation of $\varphi_{j}(s, \beta)$ to $D_{a, b}\left( \pm s_{0}\right)$. The functional equation (64) for $j+1$ takes the form

$$
\frac{1}{2 s} \frac{d}{d s} J(s)=0
$$

with

$$
J(s)=\mathscr{G}_{j}(-s, \tilde{\beta})-\varphi_{j}(s, \tilde{\beta})-\delta_{j, s}\left(\sum_{p=0}^{\mu} \frac{\left\langle\tilde{\mathscr{E}}_{p}^{(\mu)}\left(v_{s}^{(p+1)}\right) \mid \tilde{\beta}\right\rangle}{2 v_{s}^{(p+1)}}\right)
$$

on $D_{a, b}\left( \pm s_{0}\right)$. Hence $J(s)$ is a constant on the domain $D_{a, b}\left( \pm s_{0}\right)$ on the one hand. On the other hand, by the functional equation of $\tilde{\mathscr{E}}_{p}^{(\mu)}(v)$ (Lemma 5.6) and that of $v_{s}^{(p+1)}$ (Lemma 3.1), we have $J(-s)=-J(s)$. Hence the constant $J(s)$ should be zero. This establishes the functional equation (64) on $D_{a, b}\left( \pm s_{0}\right)$. By defining the value of $\mathscr{G}_{j}(s, \beta)$ for $\operatorname{Re} s<0$ by the functional equation (64), we obtain the meromorphic continuation of $\varphi_{j}(s, \beta)$ to $\mathbb{C}-L_{1}$ keeping (64) correct. The assertion on the poles for $\mathscr{G}_{j}(s, \beta)$ follows from that for $\mathscr{G}_{j+1}(s, \beta)$ from (66).

Lemma 5.8. Let $1 \leqslant p \leqslant r$ and put $v_{s}=v_{s}^{(p)}$. Let $U$ be an open domain in $\mathbb{C}-L_{1}$ and $F(z)$ a holomorphic function on some open neighborhood of $\left\{v_{s}^{(p)} \mid s \in U\right\}$. Then for each $j \geqslant 1$ we have

$$
\operatorname{Res}_{z=v_{s}} \frac{F(z)}{\left(z^{2}-v_{s}^{2}\right)^{j}}=\delta_{j-1, s}\left(\frac{F\left(v_{s}\right)}{2 v_{s}}\right), \quad s \in U .
$$

\section{Green currents}

For $\operatorname{Re} s>n$, the currents $G_{s}:=\left(c_{r} \mu \mu !\right)^{-1} P\left(\phi_{s}\right)$ and $\Psi_{s}=\left(c_{r} \mu \mu !\right)^{-1} P\left(\psi_{s}\right)$ are of $(\mu, \mu)$-type and of $(r, r)$-type respectively. In this section we study some of the properties of the currents $G_{s}$ and $\Psi_{s}$ using the knowledge of the function $\tilde{G}_{s}$ 
obtained in the previous section. We put the Kähler form $\omega$ on $\Gamma \backslash G / K$ such that $\tilde{\boldsymbol{\omega}}(g)=\omega$ for all $g \in G$. The metric on $\Gamma \backslash G / K$ corresponding to $\omega$ defines the Laplacian $\triangle$, the Lefschetz operator and its adjoint $\Lambda$ acting on the space of forms and currents on $\Gamma \backslash G / K$.

Currents defined by modular cycles. Denote by $D$ the image of the map from $\Gamma_{H} \backslash H / K_{H}$ to $\Gamma \backslash G / K$ induced by the natural holomorphic inclusion of $H / K_{H}$ into $G / K$. Then $D$, a closed complex analytic subset of $\Gamma \backslash G / K$, defines an $(r, r)$ current $\delta_{D}$ on $\Gamma \backslash G / K$ by the integration

$$
\left\langle\delta_{D}, \alpha\right\rangle=\int_{D_{\mathrm{ns}}} j^{*} \alpha, \quad \alpha \in A_{\mathrm{c}}(\Gamma \backslash G / K) .
$$

Here $j: D \hookrightarrow \Gamma \backslash G / K$ is the natural inclusion and $D_{\mathrm{ns}}$ is the smooth locus of $D$. Since $\delta_{D}$ is closed, it defines a cycle on $\Gamma \backslash G / K$ of real codimension $2 r$ [Griffiths and Harris 1978, p. 32-33].

Proposition 6.1. For $\alpha \in A_{\mathrm{c}}(\Gamma \backslash G / K)$, we have

$$
\begin{aligned}
& \left\langle\delta_{D}, * \bar{\alpha}\right\rangle=\left(* \operatorname{vol}_{H} \mid \mathscr{\Phi}_{H}(\tilde{\alpha} ; e)\right), \\
& \left\langle\Lambda \delta_{D}, * \bar{\alpha}\right\rangle=\left(\Lambda\left(* \operatorname{vol}_{H}\right) \mid \mathscr{F}_{H}(\tilde{\alpha} ; e)\right) .
\end{aligned}
$$

Proof. We give a proof assuming the natural map $p: \Gamma_{H} \backslash H / K_{H} \rightarrow \Gamma \backslash G / K$ is one-to-one. (The general case is similar.) For any $\beta \in A_{c}(\Gamma \backslash G / K)$, we have

$$
\left\langle\delta_{D}, \beta\right\rangle=\int_{\Gamma_{H} \backslash H / K_{H}} p^{*} \beta=\int_{\Gamma_{H} \backslash H}\left(f(h) \mid \operatorname{vol}_{H}\right) d \dot{h},
$$

noting that $\left\|\operatorname{vol}_{H}\right\|=1$. Here $f: H \rightarrow \bigwedge(\mathfrak{h} \cap \mathfrak{p})_{\mathbb{C}}^{*}$ is the function on $H$ corresponding to $p^{*} \beta$ which is determined by a formula similar to (48). Put $\beta=* \bar{\alpha}$. Equation (67) holds since

$$
\left(f(h) \mid \operatorname{vol}_{H}\right)=\left(\tilde{\beta}(h) \mid \operatorname{vol}_{H}\right)=\left(\operatorname{vol}_{H} \mid * \tilde{\alpha}(h)\right)=\left(* \operatorname{vol}_{H} \mid \tilde{\alpha}(h)\right) .
$$

Thus (68) follows from (67), since $\left\langle\Lambda \delta_{D}, \beta\right\rangle=\left\langle\delta_{D}, \Lambda \beta\right\rangle$ by definition.

Differential equations. First we show that $G_{s}$ and $\Psi_{s}$ satisfy some differential equations.

Theorem 6.2. Let $\operatorname{Re} s>n$. Then

$$
\begin{gathered}
\left(\triangle+s^{2}-\lambda^{2}\right) G_{s}=-4 \Lambda \delta_{D}, \\
\triangle \Psi_{s}=\left(\lambda^{2}-s^{2}\right)\left(\Psi_{s}-2 \sqrt{-1} \delta_{D}\right), \\
\partial \bar{\partial} G_{s}=\Psi_{s}-2 \sqrt{-1} \delta_{D} .
\end{gathered}
$$


Proof. It suffices to prove these formulas for $s$ with $\operatorname{Re} s>3 n-2 r$ because they depend on $s$ holomorphically; see Proposition 4.4. Since $R_{\Omega} \tilde{\alpha}=-(\triangle \alpha)^{\sim}$ for $\alpha \in A(\Gamma \backslash G / K)$ by Kuga's formula, equation (69) follows from Theorem 4.5, the first equality in (7) and (68). To prove (71), take an arbitrary form $\alpha \in A_{\mathcal{c}}(\Gamma \backslash G / K)$. First by definition and then by an application of (50),

$$
\begin{aligned}
\left\langle\partial \bar{\partial} G_{s}, * \bar{\alpha}\right\rangle & =-\left\langle G_{s}, \bar{\partial} \partial * \bar{\alpha}\right\rangle=\left\langle G_{s}, * \partial^{*} \bar{\partial}^{*} \bar{\alpha}\right\rangle=\left\langle G_{s}, * \overline{\bar{\partial}}{ }^{*} \partial^{*} \alpha\right\rangle \\
& =\frac{1}{c_{r} \mu \mu !} \int_{0}^{\infty} \varrho(t)\left(\phi_{s}\left(a_{t}\right) \mid \mathscr{F}_{H}\left(f ; a_{t}\right)\right) d t,
\end{aligned}
$$

with $f(g)=\left(\bar{\partial}^{*} \partial^{*} \alpha\right)^{\sim}(g)$. Since

$$
\left(\bar{\partial}^{*} \partial^{*} \alpha\right)^{\sim}(g)=-\sum_{\alpha, \beta=0}^{n-1} e\left(\omega_{\alpha}\right)^{*} e\left(\bar{\omega}_{\beta}\right)^{*} R_{X_{\alpha} \bar{X}_{\beta}} \tilde{\alpha}(g),
$$

we have

$$
\mathscr{F}_{H}\left(f ; a_{t}\right)=-\sum_{\alpha, \beta=0}^{n-1} e\left(\omega_{\alpha}\right)^{*} e\left(\bar{\omega}_{\beta}\right)^{*} R_{X_{\alpha} \bar{X}_{\beta}} \mathscr{F}_{H}\left(\tilde{\alpha} ; a_{t}\right),
$$

using Proposition 4.3(1). Inserting the formulas in Lemma 2.1 to the right-hand side of (73), we obtain $\mathscr{F}_{H}\left(f ; a_{t}\right)=\mathscr{E}_{t} \mathscr{\mathscr { F }}_{H}\left(f ; a_{t}\right)$ with $\mathscr{E}_{t}$ the differential operator on $t>0$ given by

$$
\begin{aligned}
\mathscr{E}_{t}=-\frac{e^{*}\left(\omega_{0} \wedge \bar{\omega}_{0}\right)}{4} \frac{d^{2}}{d t^{2}}+ & \frac{1}{2}\left(\tilde{\mathscr{A}} \tanh t+\tilde{\mathscr{P}}_{\sin } \operatorname{coth}\right) \frac{d}{d t} \\
& -\tilde{\mathscr{P}}_{+} \tilde{\mathscr{P}}_{-} \tanh ^{2} t-\tilde{\mathscr{R}}_{+} \tilde{\mathscr{R}}_{-} \operatorname{coth}^{2} t-\tilde{\mathscr{P}}_{+} \tilde{\mathscr{R}}_{-}-\tilde{\mathscr{R}}_{+} \tilde{\mathscr{P}}_{-},
\end{aligned}
$$

where

$$
\begin{gathered}
\tilde{\mathscr{P}}_{+}=\sum_{i=1}^{n-r} e^{*}\left(\bar{\omega}_{i}\right) \tau\left(Z_{i}\right), \quad \tilde{\mathscr{P}}_{-}=\sum_{i=1}^{n-r} e^{*}\left(\omega_{i}\right) \tau\left(\bar{Z}_{i}\right), \\
\tilde{\mathscr{R}}_{+}=\sum_{j=n-r+1}^{n-1} e^{*}\left(\bar{\omega}_{j}\right) \tau\left(Z_{j}\right), \quad \tilde{\mathscr{R}}_{-}=\sum_{j=n-r+1}^{n-1} e^{*}\left(\omega_{j}\right) \tau\left(\bar{Z}_{j}\right), \\
\tilde{\mathscr{A}}=e^{*}\left(2 \sqrt{-1} \omega_{H}\right)-\frac{1}{2} e^{*}\left(\omega_{0} \wedge \bar{\omega}_{0}\right)+e^{*}\left(\omega_{0}\right) \tilde{\mathscr{P}}_{+}-e^{*}\left(\bar{\omega}_{0}\right) \tilde{\mathscr{P}}_{-}, \\
\tilde{\mathscr{P}}=e^{*}(2 \sqrt{-1} \eta)-\frac{1}{2} e^{*}\left(\omega_{0} \wedge \bar{\omega}_{0}\right)+e^{*}\left(\omega_{0}\right) \tilde{\mathscr{R}}_{+}-e^{*}\left(\bar{\omega}_{0}\right) \tilde{\mathscr{R}}_{-} .
\end{gathered}
$$

Let $0<\varepsilon<R$. Integration by parts yields

$$
\begin{aligned}
\int_{\varepsilon}^{R} \varrho(t)\left(\phi_{s}\left(a_{t}\right) \mid \mathscr{F}_{H}\left(f ; a_{t}\right)\right) d t & \\
& =\Phi(R)-\Phi(\varepsilon)+\int_{\varepsilon}^{R} \varrho(t)\left(\mathscr{E}_{t}^{*} \phi_{s}\left(a_{t}\right) \mid \mathscr{F}_{H}\left(f ; a_{t}\right)\right) d t
\end{aligned}
$$


with

$$
\begin{array}{r}
\Phi(t)=-\frac{1}{4} \varrho(t)\left(\omega_{0} \wedge \bar{\omega}_{0} \wedge \phi_{s}\left(a_{t}\right) \mid F^{\prime}(t)\right) \\
+\frac{1}{4} \varrho^{\prime}(t)\left(\omega_{0} \wedge \bar{\omega}_{0} \wedge \phi_{s}\left(a_{t}\right) \mid F(t)\right)+\frac{1}{4} \varrho(t)\left(\omega_{0} \wedge \bar{\omega}_{0} \wedge \frac{d}{d t} \phi_{s}\left(a_{t}\right) \mid F(t)\right) \\
+\frac{1}{2} \varrho(t)\left(\left(\tilde{\mathbb{A}}^{*} \tanh t+\tilde{\mathscr{B}}^{*} \operatorname{coth} t\right) \phi_{s}\left(a_{t}\right) \mid F(t)\right) .
\end{array}
$$

Here $\mathscr{E}_{t}^{*}$ is the formal adjoint operator of $\mathscr{E}_{t}$, uniquely determined by the relation

$$
\int_{0}^{\infty}\left(\mathscr{E}_{t}^{*} \mathrm{a}(t) \mid \mathrm{b}(t)\right) \varrho(t) d t=\int_{0}^{\infty}\left(\mathrm{a}(t) \mid \mathscr{E}_{t} \mathrm{~b}(t)\right) \varrho(t) d t
$$

for any compactly supported $\left(\wedge \mathfrak{p}_{\mathbb{C}}^{*}\right)^{M}$-valued functions a $(t)$ and $\mathrm{b}(t)$ on $(0, \infty)$. We compute the limit of $\Phi(\varepsilon)$ as $\varepsilon$ going zero. By (40),

$$
\Phi(\varepsilon)=\frac{c_{r}}{2}\left(\left(e\left(\omega_{0} \wedge \bar{\omega}_{0}\right)+2 \tilde{\mathscr{S}}^{*}\right) \mathrm{w} \mid F(0)\right)(1+O(s ; \varepsilon, \varepsilon \log \varepsilon)) .
$$

Using the formulas in Lemma 2.1 and the relation $\tau(Z)^{*}=-\tau(\bar{Z})\left(Z \in \mathfrak{k}_{\mathbb{C}}\right)$, we compute to obtain

$$
\begin{aligned}
& \tilde{\mathscr{R}}_{+}^{*} e\left(\omega_{0}\right)=-e\left(2 \sqrt{-1} \eta+\mu \omega_{0} \wedge \bar{\omega}_{0}\right)+e\left(\omega_{0}\right) \mathscr{R}_{+}, \\
& \tilde{\mathscr{R}}_{-}^{*} e\left(\bar{\omega}_{0}\right)=e\left(2 \sqrt{-1} \eta+\mu \omega_{0} \wedge \bar{\omega}_{0}\right)+e\left(\bar{\omega}_{0}\right) \mathscr{R}_{-} .
\end{aligned}
$$

Using these formulas, (6) and the relations $\mathscr{R}_{ \pm} \mathrm{w}=0$, we obtain $\tilde{\mathscr{R}}_{+}^{*} e\left(\omega_{0}\right) \mathrm{w}=$ $\tilde{\mathscr{R}}_{-}^{*} e\left(\bar{\omega}_{0}\right) \mathrm{w}=0$. Hence

$$
\begin{aligned}
\tilde{\mathscr{B}}^{*} \mathrm{w} & =e(2 \sqrt{-1} \eta) \mathrm{w}-\frac{1}{2} e\left(\omega_{0} \wedge \bar{\omega}_{0}\right) \mathrm{w}+\tilde{\mathscr{R}}_{+}^{*} e\left(\omega_{0}\right) \mathrm{w}-\tilde{\mathscr{R}}_{-}^{*} e\left(\bar{\omega}_{0}\right) \mathrm{w} \\
& =2 \sqrt{-1} \eta \wedge \mathrm{w}-\frac{1}{2} \omega_{0} \wedge \bar{\omega}_{0} \wedge \mathrm{w} .
\end{aligned}
$$

From this formula and (77),

$$
\lim _{\varepsilon \rightarrow+0} \Phi(\varepsilon)=2 \sqrt{-1} c_{r}(\eta \wedge \mathrm{w} \mid F(0))=2 \sqrt{-1} c_{r} \mu \mu !\left(* \operatorname{vol}_{H} \mid F(0)\right)
$$

using (6) to obtain the second equality. We compute the limit of $\Phi(R)$ as $R$ tends to infinity by means of (41) noting Lemma 3.1. The result is, when $\operatorname{Re} s>n$,

$$
\lim _{R \rightarrow+\infty} \Phi(R)=0 .
$$

Putting (72), (74), (78) and (79) together and using (67), we finally obtain

$$
\left\langle\partial \bar{\partial} G_{s}, * \bar{\alpha}\right\rangle=-2 \sqrt{-1}\left\langle\delta_{D}, * \bar{\alpha}\right\rangle+\frac{1}{c_{r} \mu \mu !} \int_{0}^{\infty} \varrho(t)\left(\mathscr{E}_{t}^{*} \phi_{s}\left(a_{t}\right) \mid \mathscr{\Phi}_{H}\left(f ; a_{t}\right)\right) d t .
$$

To complete the proof of (71), we have only to prove that $\mathscr{E}_{t}^{*} \phi_{s}\left(a_{t}\right)=\psi_{s}\left(a_{t}\right)$ for $t>0$. Since $\psi_{s}\left(a_{t}\right)=\tilde{\mathscr{E}}_{t} \phi_{s}\left(a_{t}\right)$ with $\tilde{\mathscr{E}}_{t}$ the differential operator in $t$ given by (24) 
in the $z$-coordinate, it suffices to show $\mathscr{E}_{t}^{*}=\tilde{\mathscr{E}}_{t}$. For that purpose, we show that

$$
\int_{0}^{\infty}\left(\tilde{\mathscr{E}}_{t} \mathrm{a}(t) \mid \mathrm{b}(t)\right) \varrho(t) d t=\int_{0}^{\infty}\left(\mathscr{E}_{t}^{*} \mathrm{a}(t) \mid \mathrm{b}(t)\right) \varrho(t) d t
$$

for arbitrary compactly supported $\left(\bigwedge \mathfrak{p}_{\mathbb{C}}^{*}\right)^{M}$-valued $C^{\infty}$-functions a $(t)$ and $\mathrm{b}(t)$ on $(0, \infty)$. By the decomposition $G=H A K$, we can extend the functions a $(t)$ and $\mathrm{b}(t)$ to smooth functions $a(g)$ and $b(g)$ belonging to $C_{\tau}^{\infty}$ by the formula

$$
a\left(h a_{t} k\right)=\tau(k)^{-1} \mathrm{a}(t), \quad \text { and } \quad b\left(h a_{t} k\right)=\tau(k)^{-1} \mathrm{~b}(t), \quad h \in H, t>0, k \in K .
$$

By Lemma 4.1, (76) and the definitions of $\mathscr{E}_{t}$ and $\tilde{\mathscr{E}}_{t}$, we obtain

$$
\begin{aligned}
& \int_{H \backslash G}\left(\sum_{\alpha, \beta=0}^{n-1} e\left(\bar{\omega}_{\beta}\right) e\left(\omega_{\alpha}\right) R_{X_{\alpha} \bar{X}_{\beta}} a(g) \mid b(g)\right) d \dot{g}=\int_{0}^{\infty}\left(\tilde{\mathscr{E}}_{t} \mathrm{a}(t) \mid \mathrm{b}(t)\right) \varrho(t) d t, \\
& \int_{H \backslash G}\left(a(g) \mid \sum_{\alpha, \beta=0}^{n-1} e\left(\omega_{\alpha}\right)^{*} e\left(\bar{\omega}_{\beta}\right)^{*} R_{X_{\alpha} \bar{X}_{\beta}} b(g)\right) d \dot{g}=\int_{0}^{\infty}\left(\mathscr{E}_{t}^{*} \mathrm{a}(t) \mid \mathrm{b}(t)\right) \varrho(t) d t .
\end{aligned}
$$

The left-hand sides of these two formulas are easily seen to be identical, since

$\int_{H \backslash G}\left(a_{1}(g) \mid R_{X} a_{2}(g)\right) d \dot{g}=-\int_{H \backslash G}\left(R_{\bar{X}} a_{1}(g) \mid a_{2}(g)\right) d \dot{g}, \quad a_{1}, a_{2} \in C_{\tau}^{\infty}, X \in \mathfrak{g}_{\mathbb{C}}$.

We now deduce (70) from (69) and (71). That the current $\delta_{D}$ is real and closed implies that $\partial \delta_{D}=0$ and $\bar{\partial} \delta_{D}=0$. Hence $\triangle \delta_{D}=\mathrm{d} \mathrm{d}^{*} \delta_{D}=(\partial+\bar{\partial})\left(\partial^{*}+\bar{\partial}^{*}\right) \delta_{D}$. Since $\Gamma \backslash G / K$ is a Kähler manifold, we have $\partial^{*}=\sqrt{-1}[\Lambda, \bar{\partial}]$ and $\bar{\partial}^{*}=-\sqrt{-1}[\Lambda, \partial]$; see [Wells 1980, (4.5), Corollary 4.10, p. 193]. Using these equalities, we compute $\triangle \delta_{D}=\sqrt{-1}(\partial+\bar{\partial})(-[\Lambda, \partial-\bar{\partial}]) \delta_{D}=\sqrt{-1}(\partial+\bar{\partial})(\partial-\bar{\partial}) \Lambda \delta_{D}=-2 \sqrt{-1} \partial \bar{\partial} \Lambda \delta_{D}$ to obtain

$$
\triangle \delta_{D}+2 \sqrt{-1} \partial \bar{\partial} \Lambda \delta_{D}=0 .
$$

Since $\Psi_{s}=\partial \bar{\partial} G_{s}+2 \sqrt{-1} \delta_{D}$ by (71), we have

$$
\begin{aligned}
\triangle \Psi_{s} & =\triangle\left(\partial \bar{\partial} G_{s}+2 \sqrt{-1} \delta_{D}\right)=\partial \bar{\partial} \triangle G_{s}+2 \sqrt{-1} \triangle \delta_{D} \\
& =\partial \bar{\partial}\left(\left(\lambda^{2}-s^{2}\right) G_{s}-4 \Lambda \delta_{D}\right)+2 \sqrt{-1} \triangle \delta_{D} \\
& =\left(\lambda^{2}-s^{2}\right) \partial \bar{\partial} G_{s}-4 \partial \bar{\partial} \Lambda \delta_{D}+2 \sqrt{-1} \triangle \delta_{D} \\
& =\left(\lambda^{2}-s^{2}\right)\left(\Psi_{s}-2 \sqrt{-1} \delta_{D}\right)+2 \sqrt{-1}\left(\triangle \delta_{D}+2 \sqrt{-1} \partial \bar{\partial} \Lambda \delta_{D}\right) \\
& =\left(\lambda^{2}-s^{2}\right)\left(\Psi_{s}-2 \sqrt{-1} \delta_{D}\right),
\end{aligned}
$$

using (69) for the third equality, (71) for the fifth and (80) for the last. 
Main theorem. Let $A_{(2)}^{p, q}(\Gamma \backslash G / K)$ be the Hilbert space of the measurable $(p, q)$ forms on $\Gamma \backslash G / K$ with the finite $L^{2}$-norm $\|\alpha\|:=\left(\int_{\Gamma \backslash G / K} \alpha \wedge * \bar{\alpha}\right)^{1 / 2}$. For each $c \in \mathbb{C}$, let $A_{(2)}^{p, q}(\Gamma \backslash G / K ; c)$ be the $c$-eigenspace of the Laplacian $\triangle$ acting on $A_{(2)}^{p, q}(\Gamma \backslash G / K)$. In particular,

$$
\mathscr{H}_{(2)}^{p, q}(\Gamma \backslash G / K):=A_{(2)}^{p, q}(\Gamma \backslash G / K ; 0)
$$

is the space of the harmonic $L^{2}$-forms of $(p, q)$-type. For each $p$, let $\mathscr{E}_{p}^{(\mu)}(v)$ be the $C^{\infty}$-form of $(\mu, \mu)$-type on $\Gamma \backslash G / K$ corresponding to the function $\tilde{\mathscr{E}}_{p}^{(\mu)}(v)$ on $G$ defined by (63). Then Theorem 5.7 immediately gives us the following theorem.

Theorem 6.3. There exists a meromorphic family of $(\mu, \mu)$-currents $G_{s}$, with $s \in$ $\mathbb{C}-L_{1}$, on $\Gamma \backslash G / K$ with the following properties.

(1) For $s \in \mathbb{C}$ with $\operatorname{Re} s>n$, the family is given by

$$
\left\langle G_{s}, * \bar{\alpha}\right\rangle=\frac{1}{(r-1) \pi^{r}} \int_{0}^{\infty} \varrho(t)\left(\phi_{s}\left(a_{t}\right) \mid \mathscr{F}_{H}\left(\tilde{\alpha} ; a_{t}\right)\right) d t, \quad \alpha \in A_{\mathrm{c}}(\Gamma \backslash G / K) .
$$

(2) A point $s_{0} \in \mathbb{C}-L_{1}$ with $\operatorname{Re} s \geqslant 0$ is a pole of $G_{s}$ if and only if there exists an $L^{2}$-form $\alpha \in A_{(2)}^{r-1, r-1}\left(\Gamma \backslash G / K ;(n-2 r+2)^{2}-s_{0}^{2}\right)$ such that

$$
\int_{D} j^{*} *(\omega \wedge \bar{\alpha}) \neq 0
$$

In this case $s_{0}$ is a simple pole with residue

$$
\operatorname{Res}_{s=s_{0}} G_{s}=\frac{2}{s_{0}} \sum_{m}\left(\int_{D} j^{*} *\left(\omega \wedge \bar{\alpha}_{m}\right)\right) \cdot \alpha_{m} .
$$

where $\left\{\alpha_{m}\right\}$ is an orthonormal basis of $A_{(2)}^{r-1, r-1}\left(\Gamma \backslash G / K ;(n-2 r+2)^{2}-s_{0}^{2}\right)$.

(3) The following functional equation holds:

$$
G_{-s}-G_{s}=\sum_{p=0}^{r-1} \frac{\mathscr{E}_{p}^{(r-1)}\left(v_{s}^{(p+1)}\right)}{2 v_{s}^{(p+1)}}, \quad s \in \mathbb{C}-L_{1} .
$$

Theorem 6.4. There exists a meromorphic family of ( $r, r)$-currents $\Psi_{s} s \in \mathbb{C}-L_{1}$, on $\Gamma \backslash G / K$ such that for $s \in \mathbb{C}$ with $\operatorname{Re} s>n$, the current $\Psi_{s}$ is given by

$$
\left\langle\Psi_{s}, * \bar{\alpha}\right\rangle=\frac{1}{(r-1) \pi^{r}} \int_{0}^{\infty} \varrho(t)\left(\psi_{s}\left(a_{t}\right) \mid \mathscr{F}_{H}\left(\tilde{\alpha} ; a_{t}\right)\right) d t, \quad \alpha \in A_{\mathrm{c}}(\Gamma \backslash G / K) .
$$

Moreover, $\Psi_{s}$ is holomorphic at $s=n-2 r+2$.

Proof. The meromorphic continuation of $\Psi_{s}$ follows from the differential equation (71) and the meromorphicity of $G_{s}$. Let $\beta$ be the residue of $G_{s}$ at $s=\lambda$. Then 
by Theorem 6.3(2), $\beta$ is a harmonic $L^{2}$-form. Moreover $\mathrm{d} \beta$ and $\mathrm{d}^{*} \beta$ are also $L^{2}$ forms due to the fact that for any $D \in \mathfrak{g}_{\mathbb{C}}$, the derivative $R_{D} \tilde{\beta}$ is square-integrable on $\Gamma \backslash G$. Then we can conclude that $\mathrm{d} \beta=0$ and $\mathrm{d}^{*} \beta=0$ using the identity

$$
0=\|\triangle \beta\|^{2}=\|\mathrm{d} \beta\|^{2}+\left\|\mathrm{d}^{*} \beta\right\|^{2} .
$$

Since the residue of the function $s \mapsto \partial \bar{\partial} G_{s}$ at its possible simple pole $s=\lambda$ is $\partial \bar{\partial} \beta=2^{-1} \sqrt{-1} \mathrm{~d}_{\mathrm{c}} \mathrm{d} \beta=0$, the function $\partial \bar{\partial} G_{s}$ is regular at $s=\lambda$. By (71), $\Psi_{s}$ is also regular at $s=\lambda$.

Definition. We define the $(r-1, r-1)$-current $\mathscr{G}$ on $\Gamma \backslash G / K$ to be the quarter of the constant term of the Laurent expansion of $G_{s}$ at $s=\lambda$, Namely, if $\left\{\alpha_{m}\right\}$ is any orthonormal basis of $\mathcal{H}_{(2)}^{r-1, r-1}(\Gamma \backslash G / K)$, we put

$$
\mathscr{G}(x)=\frac{1}{4} \lim _{s \rightarrow \lambda}\left(G_{s}(x)-\frac{2}{n-2 r+2} \sum_{m} \int_{D} j^{*} *\left(\omega \wedge \bar{\alpha}_{m}\right) \frac{\alpha_{m}(x)}{s-(n-2 r+2)}\right) .
$$

Theorem 6.5. We have the equation

$$
\operatorname{dd}_{\mathrm{c}} \varphi=\frac{\sqrt{-1}}{2} \Psi_{n-2 r+2}+\delta_{D}, \quad \triangle \Psi_{n-2 r+2}=0 .
$$

The current $\Psi_{n-2 r+2}$ is represented by an element of $A^{r, r}(\Gamma \backslash G / K)$.

Proof. Since $\Psi_{s}$ is regular at $s=\lambda$, the differential equation (70) gives us $\Delta \Psi_{\lambda}=0$. The current $\Psi_{\lambda}$, which is annihilated by the elliptic differential operator $\Delta$ on $\Gamma \backslash G / K$, is then a $C^{\infty}$-form by the elliptic regularity theorem. By comparing the constant terms of the Laurent expansion at $s=\lambda$ of both sides of the identity (71), we obtain the first equation in the theorem.

\section{Square-integrability of $\Psi_{\lambda}$}

In this section we prove the square-integrability of $\Psi_{\lambda}$. To establish it we need the spectral expansion of the functions

$$
\boldsymbol{\delta}_{j, s}\left(\left(s^{2}-\lambda^{2}\right)^{-1} \widetilde{\Psi}_{s}\right):=\left(c_{r} \mu \mu !\right)^{-1} \tilde{P}\left(\boldsymbol{\delta}_{j, s}\left(\left(s^{2}-\lambda^{2}\right)^{-1} \psi_{s}\right)\right) .
$$

Lemma 7.1. Let $\mathrm{c}_{\alpha}(s)(0 \leqslant \alpha \leqslant \mu-1)$ be the functions appearing as coefficients in the asymptotic formula (44). For each $\alpha$ there exists an even polynomial function $\tilde{\mathrm{c}}_{\alpha}(s)$ with degree no more than $2 \alpha$ such that $\mathrm{c}_{\alpha}(s)=\left(s^{2}-\lambda^{2}\right) \tilde{\mathrm{c}}_{\alpha}(s)$. We have $\tilde{\mathrm{c}}_{0}(s)=-(\sqrt{-1} / 2) \mu ! * \mathrm{vol}_{H}$, independent of $s$. For each $0 \leqslant j \leqslant \mu$, we have

$$
\boldsymbol{\delta}_{j, s}\left(\frac{\psi_{s}\left(a_{t}\right)}{s^{2}-\lambda^{2}}\right)=\frac{\tilde{w}_{\mu-j}}{z^{\mu-j}}(1+O(s ; z, z \log z)), \quad z=\tanh ^{2} t,
$$

with $\tilde{\mathrm{w}}_{\alpha}:=\left(\boldsymbol{\delta}_{\alpha, s} \tilde{\mathrm{c}}_{\alpha}\right)(0)$. 
Proof. For $f \in \mathscr{K}_{\Gamma}(\tau)$, consider the integral

$$
I(f)=\int_{\Gamma \backslash G}\left(\left(s^{2}-\lambda^{2}\right)^{-1} \widetilde{\Psi}_{s}(g) \mid R_{\Omega+\lambda^{2}-\bar{s}^{2}} f(g)\right) d \dot{g} .
$$

By (70) and (67), $I(f)=-2 \sqrt{-1}\left(* \operatorname{vol}_{H} \mid \mathscr{F}_{H}(f ; e)\right)$ on the one hand. On the other hand, we can compute $I(f)$ in a way similar to that in the proof of Theorem 4.5 to obtain

$$
I(f)=\frac{4}{\mu !}\left(s^{2}-\lambda^{2}\right)^{-1}\left(\mathrm{c}_{0}(s) \mid \mathscr{\Phi}_{H}(f ; e)\right) .
$$

Comparing these two expressions for $I(f)$, we get

$$
-2 \sqrt{-1}\left(* \operatorname{vol}_{H} \mid \mathscr{F}_{H}(f ; e)\right)=\frac{4}{\mu !}\left(s^{2}-\lambda^{2}\right)^{-1}\left(\mathrm{c}_{0}(s) \mid \mathscr{\Phi}_{H}(f ; e)\right) .
$$

By choosing $f$ suitably, we can arrange for the value of $\mathscr{F}_{H}(f ; e)$ to be any element of $\left(\bigwedge^{r, r} \mathfrak{p}_{\mathbb{C}}^{*}\right)^{M}$. Hence the relation (82) implies

$$
\mathrm{c}_{0}(s)=-\frac{\sqrt{-1}}{2} \mu ! * \operatorname{vol}_{H}\left(s^{2}-\lambda^{2}\right) \text {. }
$$

Since the Schmid operators $\nabla_{ \pm}$commute with the Casimir operator $\Omega$, (26) implies that $\psi_{s}$ is also a $C^{\infty}$-solution of (22). Hence the same argument as in the proof of Theorem 3.14 yields that the coefficients $\mathrm{c}_{\alpha}(s)$ must obey the same recurrence relation (43) as $\mathrm{a}_{\alpha}(s)$. Since the operator $\mathscr{S}^{\prime}-\alpha(\mu-\alpha)$ is invertible on $\left(\bigwedge^{r, r} \mathfrak{p}_{\mathbb{C}}^{*}\right)^{M}$, the recurrence relation (43) with the initial condition (83) implies the first and the second assertions in the lemma. The last assertion follows from the expression $\mathrm{c}_{\alpha}(s)=\left(s^{2}-\lambda^{2}\right) \tilde{\mathrm{c}}_{\alpha}(s)$ just obtained.

Theorem 7.2. Let $0 \leqslant j \leqslant \mu$ be an integer. Suppose $\operatorname{Re} s>3 n-2 r$. Then for any $f \in \mathfrak{M}_{\delta}$ with $\delta \in\left(2 r n^{-1}, 1\right)$, we have

$\int_{\Gamma \backslash G}\left(\delta_{j, s}\left(\left(s^{2}-\lambda^{2}\right)^{-1} \widetilde{\Psi}_{s}\right)(g) \mid R_{\left(\Omega+\lambda^{2}-\bar{s}^{2}\right)^{j+1}} f(g)\right) d \dot{g}=-2 \sqrt{-1}\left(* \operatorname{vol}_{H} \mid \mathscr{F}_{H}(f ; e)\right)$.

Proof. The proof is analogous to that of Theorem 4.5. We use (81).

Theorem 7.3. Let $\operatorname{Re} s>3 n-2 r$. There exists $\varepsilon>0$ such that the function $\boldsymbol{\delta}_{\mu, s}\left(\left(s^{2}-\lambda^{2}\right)^{-1} \widetilde{\Psi}_{s}\right)$ belongs to the space $\mathscr{L}_{\Gamma}^{2+\varepsilon}(\tau)^{(r)}$. The spectral expansion of $\boldsymbol{\delta}_{\mu, s}\left(\left(s^{2}-\lambda^{2}\right)^{-1} \widetilde{\Psi}_{s}\right)$ is

$$
\boldsymbol{\delta}_{\mu, s}\left(\frac{\widetilde{\Psi}_{s}}{s^{2}-\lambda^{2}}\right)=\mathscr{F}_{\mathrm{dis}}(s)+\sum_{p=0}^{r} \mathscr{F}_{\mathrm{c}}^{(p)}(s),
$$


with

$$
\begin{aligned}
& \mathscr{F}_{\text {dis }}(s)=\sum_{m=0}^{\infty} \frac{-2 \sqrt{-1}\left(* \operatorname{vol}_{H} \mid \mathscr{F}_{H}\left(\tilde{\alpha}_{m}^{(r)} ; e\right)\right)}{\left(\lambda^{2}-\lambda_{m}^{(r)}-s^{2}\right)^{r}} \tilde{\alpha}_{m}^{(r)}, \\
& \mathscr{F}_{\mathrm{c}}^{(p)}(s)=\frac{1}{4 \pi \sqrt{-1}} \int_{\sqrt{-1} \mathbb{R}} \sum_{i=1}^{h} \sum_{\mathrm{u}} \frac{-2 \sqrt{-1}\left(* \operatorname{vol}_{H} \mid \mathscr{F}_{H}\left(\mathrm{E}^{i}(\zeta ; \mathrm{u}) ; e\right)\right)}{\left(\zeta^{2}-\left(\nu_{s}^{(p+1)}\right)^{2}\right)^{r}} \mathrm{E}^{i}(\zeta ; \mathrm{u}) d \zeta,
\end{aligned}
$$

where the inner summation is over $\mathrm{u} \in \mathscr{P}_{i}^{(r)}(p ; 0)$ and both summations are convergent in $\mathscr{L}_{\Gamma}^{2}(\tau)^{(r)}$.

Proof. Like that of Theorem 5.3. We use Theorem 7.2.

Theorem 7.4. Let $L_{1}$ be the interval on the imaginary axis defined by (25). Let $0 \leqslant j \leqslant \mu$. Then for each $\tilde{\beta} \in \mathscr{K}_{\Gamma}(\tau)$ the holomorphic function

$$
s \mapsto \mathscr{F}_{j}(s, \tilde{\beta}):=\left\langle\boldsymbol{\delta}_{j, s}\left(s^{2}-\lambda^{2}\right)^{-1} \widetilde{\Psi}_{s} \mid \tilde{\beta}\right\rangle
$$

on $\operatorname{Re} s>n$ has a meromorphic continuation to the domain $\mathbb{C}-L_{1}$. A point $s_{0} \in \mathbb{C}-L_{1}$ with $\operatorname{Re} s_{0} \geqslant 0$ is a pole of the meromorphic function $\mathscr{F}_{j}(s, \beta)$ if and only if there exists an $m \in \mathbb{N}$ such that

$$
\left(* \operatorname{vol}_{H} \mid \mathscr{F}_{H}\left(\tilde{\alpha}_{m}^{(r)} ; e\right)\right) \neq 0, \quad\left\langle\tilde{\alpha}_{m}^{(r)} \mid \tilde{\beta}\right\rangle \neq 0,
$$

and $s_{0}^{2}-\lambda^{2}=-\lambda_{m}^{(r)}$. In this case, the function

$$
\mathscr{F}_{j}(s, \beta)-\sum_{\substack{m \in \mathbb{N} \\ \lambda_{m}^{(r)}=\lambda^{2}-s_{0}^{2}}} \frac{2 \sqrt{-1}\left(* \operatorname{vol}_{H} \mid \mathscr{F}_{H}\left(\tilde{\alpha}_{m}^{(r)} ; e\right)\right)\left\langle\tilde{\alpha}_{m}^{(r)} \mid \tilde{\beta}\right\rangle}{\left(s_{0}^{2}-s^{2}\right)^{j+1}}
$$

is holomorphic at $s=s_{0}$. We have the functional equation

$$
\mathscr{F}_{j}(-s, \tilde{\beta})-\mathscr{F}_{j}(s, \tilde{\beta})=\boldsymbol{\delta}_{j, s}\left(\sum_{p=0}^{r} \frac{\left\langle\tilde{\mathscr{E}}_{p}^{(r)}\left(v_{s}^{(p+1)}\right) \mid \tilde{\beta}\right\rangle}{2 v_{s}^{(p+1)}}\right),
$$

where, for $g \in G$,

$$
\tilde{\mathscr{E}}_{p}^{(r)}(v ; g):=-2 \sqrt{-1} \sum_{i=1}^{h} \sum_{\mathrm{u} \in \mathscr{S}_{i}^{(r)}(p ; 0)}\left(* \operatorname{vol}_{H} \mid \mathscr{F}_{H}\left(\mathrm{E}^{i}(-\bar{v} ; \mathrm{u}) ; e\right)\right) \mathrm{E}^{i}(v ; \mathrm{u} ; g) .
$$

Proof. The proof is the same as that of Theorem 5.7. The statement for $j=\mu$ follows from Theorem 7.3. Then we use induction to show the statement for $j$ smaller than $\mu$. 


\section{Some properties of the current $\Psi_{s}$.}

Theorem 7.5. (1) A point $s_{0} \in \mathbb{C}-L_{1}$ with $\operatorname{Re} s_{0} \geqslant 0$ and $s_{0} \neq n-2 r+2$ is a pole of the current $\Psi_{s}$ if and only if there exists an $L^{2}$-form

$$
\alpha \in A_{(2)}^{r, r}\left(\Gamma \backslash G / K ;(n-2 r+2)^{2}-s_{0}^{2}\right)
$$

such that

$$
\int_{D} j^{*} * \alpha \neq 0
$$

In this case $s_{0}$ is a simple pole with residue

$$
\operatorname{Res}_{s=s_{0}} \Psi_{s}=\frac{\sqrt{-1}\left(s_{0}^{2}-(n-2 r+2)^{2}\right)}{s_{0}} \sum_{m}\left(\int_{D} j^{*} * \bar{\alpha}_{m}\right) \cdot \alpha_{m},
$$

where $\left\{\alpha_{m}\right\}$ is an orthonormal basis of $A_{(2)}^{r, r}\left(\Gamma \backslash G / K ;(n-2 r+2)^{2}-s_{0}^{2}\right)$.

(2) We have

$$
\Psi_{n-2 r+2}=2 \sqrt{-1} \sum_{m}\left(\int_{D} j^{*} * \bar{\beta}_{m}\right) \cdot \beta_{m},
$$

with $\left\{\beta_{m}\right\}$ an orthonormal basis of $\mathscr{H}_{(2)}^{r, r}(\Gamma \backslash G / K)$. In particular, the current $\Psi_{n-2 r+2}$ is in $\mathscr{H}_{(2)}^{r, r}(\Gamma \backslash G / K)$.

Proof. This is a corollary of Theorem 7.4.

By Theorem 6.5, the fundamental class $\left[\delta_{D}\right] \in \mathrm{H}^{r, r}(\Gamma \backslash G / K ; \mathbb{C})$ of $D$ has the harmonic $L^{2}$-representative $\Psi_{n-2 r+2}$.

\section{References}

[Borel and Wallach 1980] A. Borel and N. R. Wallach, Continuous cohomology, discrete subgroups, and representations of reductive groups, Annals of Mathematics Studies 94, Princeton University Press, Princeton, NJ, 1980. MR 83c:22018 Zbl 0443.22010

[Gillet and Soulé 1990] H. Gillet and C. Soulé, "Arithmetic intersection theory", Inst. Hautes Études Sci. Publ. Math. 72 (1990), 93-174 (1991). MR 92d:14016 Zbl 0741.14012

[Gon and Tsuzuki 2002] Y. Gon and M. Tsuzuki, "The resolvent trace formula for rank one Lie groups”, Asian J. Math. 6:2 (2002), 227-252. MR 2003j:22011 Zbl 1026.22010

[Griffiths and Harris 1978] P. Griffiths and J. Harris, Principles of algebraic geometry, Wiley, New York, 1978. MR 95d:14001 Zbl 0408.14001

[Heckman and Schlichtkrull 1994] G. Heckman and H. Schlichtkrull, Harmonic analysis and special functions on symmetric spaces, Perspectives in Mathematics 16, Academic Press, San Diego, CA, 1994. MR 96j:22019 Zbl 0836.43001

[Hejhal 1983] D. A. Hejhal, The Selberg trace formula for PSL(2, R). Vol. 2, Lecture Notes in Mathematics 1001, Springer, Berlin, 1983. MR 86e:11040 Zbl 0543.10020

[Magnus et al. 1966] W. Magnus, F. Oberhettinger, and R. P. Soni, Formulas and theorems for the special functions of mathematical physics, Third enlarged edition. Die Grundlehren der mathematischen Wissenschaften, Band 52, Springer, New York, 1966. MR 38 \#1291 Zbl 0143.08502 
[Miatello and Wallach 1989] R. Miatello and N. R. Wallach, "Automorphic forms constructed from Whittaker vectors", J. Funct. Anal. 86:2 (1989), 411-487. MR 91b:11065 Zbl 0692.10029

[Miatello and Wallach 1992] R. Miatello and N. R. Wallach, "The resolvent of the Laplacian on locally symmetric spaces”, J. Diff. Geom. 36:3 (1992), 663-698. MR 93i:58160 Zbl 0766.53044

[Oda and Tsuzuki 2003] T. Oda and M. Tsuzuki, "Automorphic Green functions associated with the secondary spherical functions”, Publ. Res. Inst. Math. Sci. 39:3 (2003), 451-533. MR 2004f:11046 Zbl 1044.11033

[Wells 1980] R. O. Wells, Jr., Differential analysis on complex manifolds, Graduate Texts in Mathematics 65, Springer, New York, 1980. MR 83f:58001 Zbl 0435.32004

Received December 15, 2004. Revised October 16, 2005.

MASAO TSUZUKI

DEPARTMENT OF MATHEMATICS

SOPHIA UNIVERSITY

KIOI-CHO 7-1 CHIYODA-KU

TOKYO, 102-8554

JAPAN

tsuzuki@mm.sophia.ac.jp 